\title{
On the Dirac-Frenkel Variational Principle on Tensor Banach Spaces
}

\author{
Antonio Falcó*,1,3, Wolfgang Hackbusch ${ }^{2}$ and Anthony Nouy ${ }^{3}$ \\ 1 ESI International Chair@CEU-UCH, \\ Departamento de Matemáticas, Física y Ciencias Tecnológicas, \\ Universidad Cardenal Herrera-CEU, CEU Universities \\ San Bartolomé 55, 46115 Alfara del Patriarca (Valencia), Spain \\ e-mail: afalco@uchceu.es \\ ${ }^{2}$ Max-Planck-Institut Mathematik in den Naturwissenschaften \\ Inselstr. 22, D-04103 Leipzig, Germany \\ e-mail: wh@mis.mpg.de \\ ${ }^{3}$ Centrale Nantes, \\ LMJL UMR CNRS 6629 \\ 1 rue de la Noë, 44321 Nantes Cedex 3, France. \\ e-mail: anthony.nouy@ec-nantes.fr
}

\begin{abstract}
The main goal of this paper is to extend the so-called Dirac-Frenkel Variational Principle in the framework of tensor Banach spaces. To this end we observe that a tensor product of normed spaces can be described as a union of disjoint connected components. Then we show that each of these connected components, composed by tensors in Tucker format with a fixed rank, is a Banach manifold modelled in a particular Banach space, for which we provide local charts. The description of the local charts of these manifolds is crucial for an algorithmic treatment of high-dimensional partial differential equations and minimization problems. In order to describe the relationship between these manifolds and the natural ambient space we prove under natural conditions that each connected component can be immersed in a particular ambient Banach space. This fact allows us to finally extend the Dirac-Frenkel variational principle in the framework of topological tensor spaces.
\end{abstract}

2010 AMS Subject Classifications: 15A69, 46B28, 46A32.

Key words: Tensor spaces, Banach manifolds, Tensor formats, Tensor rank.

\section{Introduction}

Tensor approximation methods play a central role in the numerical solution of high-dimensional problems arising in a wide range of applications. Low-rank tensor formats based on subspaces are widely used for complexity reduction in the representation of high-order tensors. The construction of these formats are usually based on a hierarchy of tensor product subspaces spanned by orthonormal bases, because in most cases a hierarchical representation fits with the structure of the mathematical model and facilitates its computational implementation. Two of the most popular formats are the Tucker format and the Hierarchical Tucker format [18 (HT for short). It is possible to show that the Tensor Train format 31] (TT for short), introduced originally by Vidal [37, is a particular case of the HT format (see e.g. Chapter 12 in [19]). An important feature of these formats, in the framework of topological tensor spaces, is the existence of a best approximation in each fixed set of tensors with bounded rank [11]. In particular, it allows us to construct,

\footnotetext{
${ }^{*}$ Corresponding author
} 
on a theoretical level, iterative minimisation methods for nonlinear convex problems over reflexive tensor Banach spaces 12 .

This paper is devoted to the use of the geometric structure of the Tucker format to construct reduced order models of ordinary differential equations defined over tensor Banach spaces. The Dirac-Frenkel variational principle is a well known tool in the numerical treatment of equations of quantum dynamics. It was originally proposed by Dirac and Frenkel in 1930 to approximately solve the time-dependent Schrödinger equation. It assumes the existence of a vector field (ordinary differential equation) over a configuration space represented by a Hilbert space. This configuration space contains an immersed submanifold and the reduced order model is then obtained by projecting the vector field at each point of the submanifold onto its tangent space. Tucker tensors of fixed rank are used in the above framework for the discretisation of differential equations arising in quantum chemical problems or in the multireference Hartree and Hartree-Fock methods (MR-HF) in quantum dynamics [27. In particular, for finite-dimensional ambient tensor spaces, it can be shown that the set of Tucker tensors of fixed rank forms an immersed finite-dimensional quotient manifold [24]. A similar approach in a complex Hilbert space setting for Tucker tensors of fixed rank is given in [4]. Then the numerical treatment of this class of problems follows the general concepts of differential equations on manifolds [16. Recently, similar results have been obtained for the TT format 22] and the HT format 35] (see also [3]). The term "matrix-product state" (MPS) was introduced in quantum physics (see, e.g., 36]). The related tensor representation can be found already in [37] without a special naming of the representation. The method has been reinvented by Oseledets and Tyrtyshnikov (see [30, 31, and [32]) and called "TT decomposition". For matrix product states (MPS), the differential geometry in a finite-dimensional complex Hilbert space setting is covered in [17. Two commonly accepted facts are the following.

(a) Even if it can be shown in finite dimension that the set of Tucker tensors with bounded rank is closed, the existence of a manifold structure for this set is an open question. Thus the existence of minimisers over this set can be shown, however, no first order optimality conditions are available from a geometric point of view.

(b) Even if either in finite dimension or in a Hilbert space setting it can be shown that the set of Tucker (respectively, in finite dimensions HT) tensors with fixed rank is a quotient manifold, an explicit parametrisation in order to provide a manifold structure is not known.

In our opinion, these two facts are due to the lack of a common mathematical framework for developing a mathematical analysis of these abstract objects. The main goal of this paper is to provide this common framework by means of some of the tools developed in 11] by some of the authors of this article in order to extend the Dirac-Frenkel variational method to the framework of tensor Banach spaces.

Our starting point are the following natural questions that arise in the mathematical theory of tensor spaces. The first question is: It is possible to construct a parametrisation for the set of tensors of fixed rank in order to show that it is a true manifold even in the infinite-dimensional case? In a second step, if the answer is positive, we would like to ask: Is the set of tensors of fixed rank an immersed submanifold of the topological tensor space, as ambient manifold, under consideration? Finally, if the above two questions have positive answers, we would like to extend the Dirac-Frenkel variational principle on tensor Banach spaces.

The paper is organised as follows.

- In Sect. 2, we introduce some important definitions and results that we will use widely along this paper. In particular we introduce Banach manifolds not modelled in a particular Banach space and we give as example the Grassmann manifold of a Banach space introduced by A. Douady [9] in 1966. Our main contribution is to give a Banach manifold structure to the set of subspaces of a normed space with a fixed finite dimension.

- In Sect. 3, we introduce the set of tensors in Tucker format with fixed rank over a tensor product space of normed spaces. We prove that if the tensor product space has a norm such that the tensor product is continuous, with respect to that norm, then the set of tensors in Tucker format with fixed rank is a $\mathcal{C}^{\infty}$-Banach manifold modelled on a particular Banach space. We point out that the regularity of the manifold depends on the regularity of the tensor product considered as a multilinear 
map between normed spaces. Even if a continuous multilinear map between complex Banach spaces is always analytic, under the authors' knowledge, for a continuous multilinear map between normed spaces we can only obtain a $\mathcal{C}^{\infty}$-differentiability. An interesting remark is that the geometric structure is independent of the choice of the norm on the tensor product space. We illustrate this fact by means of an example using Sobolev spaces. Finally, we show under the above conditions that a tensor space of normed spaces is a $\mathcal{C}^{\infty}$-Banach manifold not modelled on a particular Banach space.

- In Sect. 4, we discuss the choice of a norm in the ambient tensor Banach space to prove that the set of tensors with fixed Tucker rank is an immersed submanifold of that space (considered as Banach manifold). To this end we assume the existence of a norm over the tensor space not weaker than the injective norm . The same assumption is used in [11] to prove the existence of a best approximation in the Tucker case. Then we show that the set of tensors in Tucker format with fixed rank is an immersed submanifold of the ambient tensor Banach space. This fact is far from trivial. The main difficulty is to prove that the tangent space is a closed and complemented subspace of the ambient tensor Banach space under consideration. In a Hilbert space, every closed subspace is complemented, but this fact is not true in a Banach (non Hilbert) space.

- In Sect. 5, we give a formalisation in this framework of the multi-configuration time-dependent Hartree (MCTDH) method (see [27]) in tensor Banach spaces.

\section{The Grassmann-Banach manifold and its relatives}

In this section we introduce some important definitions and results that we will use (and elsewhere): throughout this paper.

In the following, $X$ is either a normed space or a Banach space with norm $\|\cdot\|$. We denote by $X^{*}$ the topological dual of $X$. The dual norm $\|\cdot\|_{X^{*}}$ on $X^{*}$ is

$$
\|\varphi\|_{X^{*}}=\sup \left\{|\varphi(x)|: x \in X \text { with }\|x\|_{X} \leq 1\right\}=\sup \left\{|\varphi(x)| /\|x\|_{X}: 0 \neq x \in X\right\} .
$$

We recall that if $X$ is a normed space, then $X^{*}$ is always a Banach space.

By $\mathcal{L}(X, Y)$ we denote the space of continuous linear mappings from $X$ into $Y$. The corresponding operator norm is written as $\|\cdot\|_{Y \leftarrow X}$. If $X$ and $Y$ are normed spaces then $\left(\mathcal{L}(X, Y),\|\cdot\|_{Y \leftarrow X}\right)$ is a normed space. It is well known that if $Y$ is a Banach space then $\left(\mathcal{L}(X, Y),\|\cdot\|_{Y \leftarrow X}\right)$ is also a Banach space.

Let $X_{1}, \ldots, X_{d}$ and $Y$ be normed spaces and $M: \times_{\alpha=1}^{d} X_{\alpha} \rightarrow Y$. We will say that $M$ is a multilinear map if for each fixed $\alpha \in\{1,2, \ldots, d\}$,

$$
x_{\alpha} \mapsto M\left(x_{1}, \ldots, x_{\alpha-1}, x_{\alpha}, x_{\alpha+1}, \ldots, x_{d}\right)
$$

is a linear map from $X_{\alpha}$ to $Y$ for all $\left(x_{1}, \ldots, x_{\alpha-1}, x_{\alpha+1}, \ldots, x_{d}\right) \in X_{k \in\{1,2, \ldots, d\} \backslash\{\alpha\}} X_{k}$. Recall that a multilinear map $M$ from $\chi_{\alpha=1}^{d}\left(X_{\alpha},\|\cdot\|_{\alpha}\right)$ equipped with the product topology $\|\cdot\|$ to a normed space $\left(Y,\|\cdot\|_{Y}\right)$ is continuous if and only if $\|M\|<\infty$, with

$$
\|M\|:=\sup _{\substack{\left(x_{1}, \ldots, x_{d}\right) \\\left\|x_{1}\right\|_{1} \leq 1, \ldots,\left\|x_{d}\right\|_{d} \leq 1}}\left\|M\left(x_{1}, \ldots, x_{d}\right)\right\|_{Y}=\sup _{\left(x_{1}, \ldots, x_{d}\right)} \frac{\left\|M\left(x_{1}, \ldots, x_{d}\right)\right\|_{Y}}{\left\|x_{1}\right\|_{1} \ldots\left\|x_{d}\right\|_{d}} .
$$

A useful result is the following (see Proposition 79 in [20]).

Proposition 2.1 Let $X_{1}, \ldots, X_{d}$ and $Y$ be normed spaces and $M: \times_{\alpha=1}^{d} X_{\alpha} \rightarrow Y$ be a continuous multilinear map. Then $M$ is $\mathcal{C}^{\infty}$-Fréchet differentiable and $D^{k} M\left(x_{1}, \ldots, x_{d}\right)=0$ for all $\left(x_{1}, \ldots, x_{d}\right) \in \times_{\alpha=1}^{d} X_{\alpha}$ and $k>d$. 
Assume that $X$ and $Y$ are Banach spaces and let $U \subset X$ be an open connected set. Then a map $f: U \subset X \rightarrow X$ is an analytic map in $U$ if and only if for $x \in U$ and $\varphi \in Y^{*}$, there exists a neighbourhood of 0 , namely $V(0) \subset \mathbb{K}$, where $\mathbb{K}$ is either $\mathbb{R}$ or $\mathbb{C}$, such that the map

$$
V(0) \subset \mathbb{K} \rightarrow \mathbb{K}, \quad t \mapsto \varphi(f(x+t h))
$$

is analytic. An immediate consequence of this definition is the fact that for $|t|$ sufficiently small and $x \in U$,

$$
\varphi(f(x+t h))=\sum_{n=0}^{\infty} a_{n}(x, h) \frac{t^{n}}{n !},
$$

where

$$
a_{n}(x, h)=\left.\frac{d^{n}}{d t^{n}} \varphi(f(x+t h))\right|_{t=0} .
$$

The following result characterises an analytic function defined over complex Banach spaces (see Theorem 160 in [20]).

Proposition 2.2 Let $X, Y$ be complex Banach spaces, $U \subset X$ open, and $f: U \subset X \rightarrow Y$. Then $f$ is analytic if and only if $f$ is $\mathcal{C}^{1}$-Fréchet differentiable.

Corollary 2.3 Let $X_{1}, \ldots, X_{d}$ and $Y$ be complex Banach spaces and $M: \times_{\alpha=1}^{d} X_{\alpha} \rightarrow Y$ be a continuous multilinear map. Then $M$ is analytic.

Definition 2.4 Let $X$ be a Banach space and $P \in \mathcal{L}(X, X)$. We say that $P$ is a projection if and only if $P \circ P=P$ holds. In this situation we also say that $P$ is a projection from $X$ onto $P(X):=\operatorname{Im} P$ parallel to Ker $P$.

From now on, we will denote $P \circ P=P^{2}$. Observe that if $P$ is a projection then $i d_{X}-P$ is also a projection. Moreover, $i d_{X}-P$ is parallel to $\operatorname{Im} P$.

Observe that each projection gives rise to a pair of subspaces, namely $U=\operatorname{Im} P$ and $W=\operatorname{Ker} P$ such that $X=U \oplus W$. It allows us to introduce the following definitions.

Definition 2.5 A subspace $U$ of a Banach space $X$ is said to be complemented in $X$ if there is a projection $P \in \mathcal{L}(X, X)$ from $X$ onto $U$.

Definition 2.6 Let $U$ be a closed subspace of $X$. We say that $U$ is a split subspace of $X$ if there exists $W$, called (topological) complement of $U$ in $X$, such that $X=U \oplus W$ and $W$ is a closed subspace of $X$. Moreover, we will say that $(U, W)$ is a pair of complementary subspaces of $X$.

Corresponding to each pair $(U, W)$ of complementary subspaces, there is a projection $P$ mapping $X$ onto $U$ along $W$, defined as follows. Since for each $x$ there exists a unique decomposition $x=u+w$, where $u \in U$ and $w \in W$, we can define a linear map $P(u+w):=u$, where $\operatorname{Im} P=U$ and $\operatorname{Ker} P=W$. Moreover, $P^{2}=P$. In Proposition 2.8 it will follow that $P \in \mathcal{L}(X, X)$.

Definition 2.7 The Grassmann manifold of a Banach space $X$, denoted by $\mathbb{G}(X)$, is the set of split subspaces of $X$.

$U \in \mathbb{G}(X)$ holds if and only if $U$ is a closed subspace and there exists a closed subspace $W$ in $X$ such that $X=U \oplus W$. Observe that $X$ and $\{0\}$ are in $\mathbb{G}(X)$. Moreover, by the proof of Proposition 4.2 of [10], the following result can be shown.

Proposition 2.8 Let $X$ be a Banach space. The following conditions are equivalent:

(a) $U \in \mathbb{G}(X)$.

(b) $U$ is a closed subspace and there exists $P \in \mathcal{L}(X, X)$ such that $P^{2}=P$ and $\operatorname{Im} P=U$.

(c) There exists $Q \in \mathcal{L}(X, X)$ such that $Q^{2}=Q$ and $\operatorname{Ker} Q=U$. 
Moreover, from Theorem 4.5 in [10, the following result can be shown.

Proposition 2.9 Let $X$ be a Banach space. Then every finite-dimensional subspace $U$ belongs to $\mathbb{G}(X)$.

Let $W$ and $U$ be closed subspaces of a Banach space $X$ such that $X=U \oplus W$. From now on, we will denote by $P_{U \oplus W}$ the projection onto $U$ along $W$. Then we have $P_{W \oplus U}=i d_{X}-P_{U \oplus W}$. Let $U, U^{\prime} \in \mathbb{G}(X)$. We say that $U$ and $U^{\prime}$ have a common complementary subspace in $X$ if $X=U \oplus W=U^{\prime} \oplus W$ for some $W \in \mathbb{G}(X)$. The following two results will be useful (for the first one see Lemma 2.1 in [8]).

Lemma 2.10 Let $X$ be a Banach space and assume that $W, U$, and $U^{\prime}$ are in $\mathbb{G}(X)$. Then the following statements are equivalent:

(a) $X=U \oplus W=U^{\prime} \oplus W$, i.e., $U$ and $U^{\prime}$ have a common complement in $X$.

(b) $\left.P_{U \oplus W}\right|_{U^{\prime}}: U^{\prime} \rightarrow U$ has an inverse.

Furthermore, if $Q=\left(\left.P_{U \oplus W}\right|_{U^{\prime}}\right)^{-1}$, then $Q$ is bounded and $Q=\left.P_{U^{\prime} \oplus W}\right|_{U}$.

We recall that an algebra is unital or unitary if it has an identity element with respect to the multiplication.

Proposition 2.11 Let $X$ be a Banach space and $U, W \in \mathbb{G}(X)$ be such that $X=U \oplus W$ and consider the linear space

$$
\mathcal{L}_{(U, W)}(X, X):=\left\{P_{W \oplus U} \circ S \circ P_{U \oplus W}: S \in \mathcal{L}(X, X)\right\} .
$$

Then the bounded linear map

$$
\mathcal{L}(U, W) \longrightarrow \mathcal{L}_{(U, W)}(X, X), \quad L \mapsto P_{W \oplus U} \circ L \circ P_{U \oplus W}
$$

is an isometry. Moreover, for all $L, L^{\prime} \in \mathcal{L}_{(U, W)}(X, X)$ it holds that $L \circ L^{\prime}=L^{\prime} \circ L=0$. Then $\mathcal{L}_{(U, W)}(X, X)$ is a sub-algebra of the unital Banach algebra $\mathcal{L}(X, X)$ and

$$
\exp (L)=\sum_{n=0}^{\infty} \frac{L^{n}}{n !}=i d_{X}+L \text { and } \exp (-L)=i d_{X}-L=\left(i d_{X}+L\right)^{-1}
$$

Proof. Clearly, the map is a linear isomorphism and since

$$
\|L\|_{W \leftarrow U}=\left\|P_{W \oplus U} \circ L \circ P_{U \oplus W}\right\|_{X \leftarrow X},
$$

it is an isometry. For $L=P_{W \oplus U} \circ S \circ P_{U \oplus W} \in \mathcal{L}_{(U, W)}(X, X)$ and $L^{\prime}=P_{W \oplus U} \circ S^{\prime} \circ P_{U \oplus W} \in \mathcal{L}_{(U, W)}(X, X)$ we have

$$
L \circ L^{\prime}=P_{W \oplus U} \circ S \circ P_{U \oplus W} \circ P_{W \oplus U} \circ S^{\prime} \circ P_{U \oplus W}=0,
$$

because $P_{U \oplus W} \circ P_{W \oplus U}=0$, then the second statement holds and the final statement follows in a straightforward way.

Next, we recall the definition of a Banach manifold.

Definition 2.12 Let $\mathbb{M}$ be a set. An atlas of class $C^{p}(p \geq 0)$ or analytic on $\mathbb{M}$ is a family of charts with some indexing set $A$, namely $\left\{\left(M_{\alpha}, u_{\alpha}\right): \alpha \in A\right\}$, having the following properties:

AT1 $\left\{M_{\alpha}\right\}_{\alpha \in A}$ is a covering1 of $\mathbb{M}$, that is, $M_{\alpha} \subset \mathbb{M}$ for all $\alpha \in A$ and $\cup_{\alpha \in A} M_{\alpha}=\mathbb{M}$.

AT2 For each $\alpha \in A,\left(M_{\alpha}, u_{\alpha}\right)$ stands for a bijection $u_{\alpha}: M_{\alpha} \rightarrow U_{\alpha}$ of $M_{\alpha}$ onto an open set $U_{\alpha}$ of a Banach space $X_{\alpha}$, and for any $\alpha$ and $\beta$ the set $u_{\alpha}\left(M_{\alpha} \cap M_{\beta}\right)$ is open in $X_{\alpha}$.

AT3 Finally, if we let $M_{\alpha} \cap M_{\beta}=M_{\alpha \beta}$ and $u_{\alpha}\left(M_{\alpha \beta}\right)=U_{\alpha \beta}$, the transition mapping $u_{\beta} \circ u_{\alpha}^{-1}: U_{\alpha \beta} \rightarrow U_{\beta \alpha}$ is a diffeomorphism of class $C^{p}(p \geq 0)$ or analytic.

\footnotetext{
${ }^{1}$ The condition of an open covering is not needed, see 25].
} 
Since different atlases can give the same manifold, we say that two atlases are compatible if each chart of one atlas is compatible with the charts of the other atlas in the sense of AT3. One verifies that the relation of compatibility between atlases is an equivalence relation.

Definition 2.13 An equivalence class of atlases of class $C^{p}$ on $\mathbb{M}$ is said to define a structure of a $C^{p}$ Banach manifold on $\mathbb{M}$, and hence we say that $\mathbb{M}$ is a Banach manifold. In a similar way, if an equivalence class of atlases is given by analytic maps, then we say that $\mathbb{M}$ is an analytic Banach manifold. If $X_{\alpha}$ is a Hilbert space for all $\alpha \in A$, then we say that $\mathbb{M}$ is a Hilbert manifold.

In condition AT2 we do not require that the Banach spaces are the same for all indices $\alpha$, or even that they are isomorphic. If $X_{\alpha}$ is linearly isomorphic to some Banach space $X$ for all $\alpha$, we have the following definition.

Definition 2.14 Let $\mathbb{M}$ be a set and $X$ be a Banach space. We say that $\mathbb{M}$ is a $C^{p}$ (respectively, analytic) Banach manifold modelled on $X$ if there exists an atlas of class $C^{p}$ (respectively, analytic) over $\mathbb{M}$ with $X_{\alpha}$ linearly isomorphic to $X$ for all $\alpha \in A$.

Example 2.15 Every Banach space is a Banach manifold modelled on itself (for a Banach space $Y$, simply take $\left(Y, i d_{Y}\right)$ as atlas, where $i d_{Y}$ is the identity map on $\left.Y\right)$. We would point out that the trivial linear space $\{\mathbf{0}\}$ is also a (trivial) Banach manifold modelled on itself. In particular, the set of all bounded linear maps $\mathcal{L}(X, X)$ of a Banach space $X$ is also a Banach manifold modelled on itself.

If $X$ is a Banach space, then the set of all bounded linear automorphisms of $X$ will be denoted by

$$
\mathrm{GL}(X):=\{A \in \mathcal{L}(X, X): A \text { invertible }\} .
$$

Before giving the next examples, we introduce the following definition.

Definition 2.16 Let $X$ and $Y$ be two Banach manifolds. Let $F: X \rightarrow Y$ be a map. We shall say that $F$ is $a \mathcal{C}^{r}$ (respectively, analytic) morphism if given $x \in X$ there exists a chart $(U, \varphi)$ at $x$ and a chart $(W, \psi)$ at $F(x)$ such that $F(U) \subset W$, and the map

$$
\psi \circ F \circ \varphi^{-1}: \varphi(U) \rightarrow \psi(W)
$$

is a $\mathcal{C}^{r}$-Fréchet differentiable (respectively, analytic) map.

Example 2.17 If $X$ is a Banach space, then $\mathrm{GL}(X)$ is a Banach manifold modelled on $\mathcal{L}(X, X)$, because it is an open set in $\mathcal{L}(X, X)$. Moreover, the map $A \mapsto A^{-1}$ is analytic (see 2.7 in [34]).

Example 2.18 If $X$ is a Banach space, then the exponential map exp : $\mathcal{L}(X, X) \rightarrow \operatorname{GL}(X)$ defined by $\exp (A)=\sum_{n=0}^{\infty} \frac{A^{n}}{n !}$ is an analytic map (see 2.8 in [34]).

Example 2.19 If $X$ is a Banach space, then $\mathrm{GL}(X) \times \mathrm{GL}(X)$ is a Banach manifold and the multiplication map $m: \mathrm{GL}(X) \times \mathrm{GL}(X) \rightarrow \mathrm{GL}(X)$ defined by $m(A, B)=A \circ B$ is an analytic map (see Theorem 2.42(ii) in [6] ).

Example 2.20 Let $X$ be a Banach space and $U, W \in \mathbb{G}(X)$ be such that $X=U \oplus W$. From Proposition 2.11 we know that $\mathcal{L}_{(U, W)}(X, X)$ is a sub-algebra of the Banach Algebra $\mathcal{L}(X, X)$. Then from Theorem 3.5 of [6] we have that

$$
\operatorname{GL}\left(\mathcal{L}_{(U, W)}(X, X)\right):=\left\{\exp (L): L \in \mathcal{L}_{(U, W)}(X, X)\right\} \subset \mathrm{GL}(X)
$$

is a closed Lie subgroup with associated Lie algebra $\mathcal{L}_{(U, W)}(X, X)$ and it is also an analytic Banach manifold modelled into itself. Since $\exp (L)=i d_{X}+L$ then $\exp (L)$ is a linear isomorphism between the linear subspace $U$ and $\exp (L)(U)=\left\{\left(i d_{X}+L\right)(u): u \in U\right\}$. We remark that for all $x \in X$ we have

$$
\exp (L)(x)=\exp (L)(u+w)=\exp (L)(u)+w, \quad(x=u+w, u \in U \text { and } w \in W),
$$

because $L(w)=0$, hence $\left.\exp (L)\right|_{W}=i d_{W}$ and $\left.\exp (L)\right|_{U}=i d_{U}+L$. Moreover, the maps $\exp : \mathcal{L}_{(U, W)}(X, X) \rightarrow$ $\operatorname{GL}\left(\mathcal{L}_{(U, W)}(X, X)\right), m: \operatorname{GL}\left(\mathcal{L}_{(U, W)}(X, X)\right) \times \mathrm{GL}\left(\mathcal{L}_{(U, W)}(X, X)\right) \rightarrow \mathrm{GL}\left(\mathcal{L}_{(U, W)}(X, X)\right)$ and the map $\exp (L) \mapsto$ $\exp (-L)$ are analytic. 
The next example is a Banach manifold not modelled on a particular Banach space.

Example 2.21 (Grassmann-Banach manifold) Let $X$ be a Banach space. Then, following [9] (see also [34] and [28]), it is possible to construct an atlas for $\mathbb{G}(X)$. To do this, let us denote one of the complements of $U \in \mathbb{G}(X)$ by $W$, i.e., $X=U \oplus W$. Then we define the Banach Grassmannian of $U$ relative to $W$ by

$$
\mathbb{G}(W, X):=\{V \in \mathbb{G}(X): X=V \oplus W\} .
$$

By using Lemma 2.10 it is possible to introduce a bijection

$$
\Psi_{U \oplus W}: \mathbb{G}(W, X) \longrightarrow \mathcal{L}(U, W)
$$

defined by

$$
\Psi_{U \oplus W}\left(U^{\prime}\right)=\left.\left.P_{W \oplus U}\right|_{U^{\prime}} \circ P_{U^{\prime} \oplus W}\right|_{U}=\left.P_{W \oplus U}\right|_{U^{\prime}} \circ\left(\left.P_{U \oplus W}\right|_{U^{\prime}}\right)^{-1} .
$$

It can be shown that the inverse

$$
\Psi_{U \oplus W}^{-1}: \mathcal{L}(U, W) \longrightarrow \mathbb{G}(W, X)
$$

is given by

$$
\Psi_{U \oplus W}^{-1}(L)=G(L):=\left\{\left(i d_{X}+L\right)(u): u \in U\right\} .
$$

From Proposition 2.11 we can identify $\mathcal{L}(U, W)$ with $\mathcal{L}_{(U, W)}(X, X)$. Hence can write

$$
\left(i d_{X}+L\right)=\exp (L)
$$

which following Example 2.20 can be proved to be a linear isomorphism from $U$ to $G(L)$. Observe that $G(0)=U$ and $G(L) \oplus W=X$ for all $L \in \mathcal{L}(U, W)$. Finally, to prove that this manifold is analytic we need to describe the overlap maps. To explain the behaviour of one overlap map, assume that $X=U \oplus W=U^{\prime} \oplus W^{\prime}$ and the existence of $U^{\prime \prime} \in \mathbb{G}(W, X) \cap \mathbb{G}\left(W^{\prime}, X\right)$. Let $L \in \mathcal{L}(U, W)$ and $L^{\prime} \in \mathcal{L}\left(U^{\prime}, W^{\prime}\right)$ be such that

$$
\Psi_{U \oplus W}^{-1}(L)=G(L)=U^{\prime \prime}=G\left(L^{\prime}\right)=\Psi_{U^{\prime} \oplus W^{\prime}}^{-1}\left(L^{\prime}\right) .
$$

Then it follows that

$$
X=U \oplus W=U^{\prime} \oplus W^{\prime}=G(L) \oplus W=G(L) \oplus W^{\prime} .
$$

Finally, it can be shown that the map $\left(\Psi_{U^{\prime} \oplus W^{\prime}} \circ \Psi_{U \oplus W}^{-1}\right): \mathcal{L}(U, W) \rightarrow \mathcal{L}\left(U^{\prime}, W^{\prime}\right)$ given by

$$
\left(\Psi_{U^{\prime} \oplus W^{\prime}} \circ \Psi_{U \oplus W}^{-1}\right)(L)=\Psi_{U^{\prime} \oplus W^{\prime}}(\exp (L)(U))=L^{\prime}
$$

is analytic. Then we have that the collection $\left\{\mathbb{G}(W, X), \Psi_{U \oplus W}\right\}_{U \in \mathbb{G}(X)}$ is an analytic atlas, and therefore, $\mathbb{G}(X)$ is an analytic Banach manifold. In particular, for each $U \in \mathbb{G}(X)$ the set $\mathbb{G}(W, X)$ is a Banach manifold modelled on $\mathcal{L}(U, W)$. Observe that if $U$ and $U^{\prime}$ are finite-dimensional subspaces of $X$ such that $\operatorname{dim} U \neq \operatorname{dim} U^{\prime}$ and $X=U \oplus W=U^{\prime} \oplus W^{\prime}$, then $\mathcal{L}(U, W)$ is not linearly isomorphic to $\mathcal{L}\left(U^{\prime}, W^{\prime}\right)$.

Example 2.22 Let $X$ be a Banach space. From Proposition 2.9, every finite-dimensional subspace belongs to $\mathbb{G}(X)$. It allows to introduce $\mathbb{G}_{n}(X)$, the space of all $n$-dimensional subspaces of $X(n \geq 0)$. It can be shown (see [28]) that $\mathbb{G}_{n}(X)$ is a connected component of $\mathbb{G}(X)$, and hence it is also a Banach manifold modelled on $\mathcal{L}(U, W)$, here $U \in \mathbb{G}_{n}(X)$ and $X=U \oplus W$. Moreover,

$$
\mathbb{G}_{\leq r}(X):=\bigcup_{n \leq r} \mathbb{G}_{n}(X)
$$

is also a Banach manifold for each fixed $r<\infty$.

The next example introduces the Banach-Grassmannian manifold for a normed (non-Banach) space. To the authors knowledge there is no reference in the literature about this (non-trivial) Banach manifold structure. We need the following lemma.

Lemma 2.23 Assume that $(X,\|\cdot\|)$ is a normed space and let $\bar{X}$ be the Banach space obtained as the completion of $X$. Let $U \in \mathbb{G}_{n}(\bar{X})$ be such that $U \subset X$ and $\bar{X}=U \oplus W$ for some $W \in \mathbb{G}(\bar{X})$. Then every subspace $U^{\prime} \in \mathbb{G}(W, \bar{X})$ is a subspace of $X$, that is, $U^{\prime} \subset X$. 
Proof. First of all observe that $X=U \oplus(W \cap X)$ where $W \cap X$ is a linear subspace dense in $W=$ $W \cap \bar{X}$. Assume that the lemma is not true. Then there exists $U^{\prime} \in \mathbb{G}(W, \bar{X})$ such that $U^{\prime} \oplus W=\bar{X}$ and $U^{\prime} \cap X \neq U^{\prime}$. Clearly $U^{\prime} \cap X \neq\{0\}$, otherwise $W \cap X=X$ and hence $U=\{0\}$, a contradiction. We have $X=\left(U^{\prime} \cap X\right) \oplus(W \cap X)$, which implies $\bar{X}=\left(U^{\prime} \cap X\right) \oplus W$, that is, $U^{\prime} \cap X \in \mathbb{G}(W, \bar{X})$, a contradiction with $\operatorname{dim}\left(U^{\prime} \cap X\right)<\operatorname{dim} U^{\prime}=n$. Thus the lemma follows.

Example 2.24 Assume that $(X,\|\cdot\|)$ is a normed space and let $\bar{X}$ be the Banach space obtained as the completion of $X$. We define the set $\mathbb{G}_{n}(X)$ as follows. We say that $U \in \mathbb{G}_{n}(X)$ if and only if $U \in \mathbb{G}_{n}(\bar{X})$ and $U \subset X$. Then $\mathbb{G}_{n}(X)$ is also a Banach manifold. To see this observe that, by Lemma 2.23, for each $U \in \mathbb{G}_{n}(X)$ such that $\bar{X}=U \oplus W$ for some $W \in \mathbb{G}(\bar{X})$, we have $\mathbb{G}(W, \bar{X}) \subset \mathbb{G}_{n}(X)$. Then the collection $\left\{\Psi_{U \oplus W}, \mathbb{G}(W, \bar{X})\right\}_{U \in \mathbb{G}_{n}(X)}$ is an analytic atlas on $\mathbb{G}_{n}(X)$, and therefore, $\mathbb{G}_{n}(X)$ is an analytic Banach manifold modelled on $\mathcal{L}(U, W)$, here $U \in \mathbb{G}_{n}(X)$ and $\bar{X}=U \oplus W$. Moreover, as in Example 2.22, we can define a Banach manifold $\mathbb{G}_{\leq r}(X)$ for each fixed $r<\infty$.

Let $\mathbb{M}$ be a Banach manifold of class $\mathcal{C}^{p}(p \geq 1)$ or analytic. Let $m$ be a point of $\mathbb{M}$. We consider triples $(U, \varphi, v)$ where $(U, \varphi)$ is a chart at $m$ and $v$ is an element of the vector space in which $\varphi(U)$ lies. We say that two of such triples $(U, \varphi, v)$ and $(V, \psi, w)$ are equivalent if the derivative of $\psi \circ \varphi^{-1}$ at $\varphi(m)$ maps $v$ on $w$. Thanks to the chain rule it is an equivalence relation. An equivalence class of such triples is called a tangent vector of $\mathbb{M}$ at $m$.

Definition 2.25 The set of such tangent vectors is called the tangent space of $\mathbb{M}$ at $m$ and it is denoted by $\mathbb{T}_{m}(\mathbb{M})$.

Each chart $(U, \varphi)$ determines a bijection of $\mathbb{T}_{m}(\mathbb{M})$ on a Banach space, namely the equivalence class of $(U, \varphi, v)$ corresponds to the vector $v$. By means of such a bijection it is possible to equip $\mathbb{T}_{m}(\mathbb{M})$ with the structure of a topological vector space given by the chart, and it is immediate that this structure is independent of the selected chart.

Example 2.26 If $X$ is a Banach space, then $\mathbb{T}_{x}(X)=X$ for all $x \in X$.

Example 2.27 Let $X$ be a Banach space and take $A \in \mathrm{GL}(X)$. Then $\mathbb{T}_{A}(\mathrm{GL}(X))=\mathcal{L}(X, X)$.

Example 2.28 For $U \in \mathbb{G}(X)$ such that $X=U \oplus W$ for some $W \in \mathbb{G}(X)$, we have $\mathbb{T}_{U}(\mathbb{G}(X))=\mathcal{L}(U, W)$.

Example 2.29 For a Hilbert space $X$ with associated inner product $\langle\cdot, \cdot\rangle$ and norm $\|\cdot\|$, its unit sphere denoted by

$$
\mathbb{S}_{X}:=\{x \in X:\|x\|=1\}
$$

is a Hilbert manifold of co-dimension one. Moreover, for each $x \in \mathbb{S}_{X}$, its tangent space is

$$
\mathbb{T}_{x}\left(\mathbb{S}_{X}\right)=\operatorname{span}\{x\}^{\perp}=\left\{x^{\prime} \in X:\left\langle x, x^{\prime}\right\rangle=0\right\} .
$$

\section{The manifold of tensors in Tucker format with fixed rank}

The MCTDH method is based on the construction of approximations of the wave function which, at every time $t$, lie in the algebraic tensor space ${ }_{a} \bigotimes_{\alpha=1}^{d} V_{\alpha}$ where $V_{\alpha}=L^{2}\left(\mathbb{R}^{3}\right)$ for $\alpha=1,2, \ldots, d$ (see [27]). Clearly, this set is a linear space. However it is not clear whether or not it is a (Hilbert/Banach) manifold, because it is a dense subspace of the Hilbert tensor space $L^{2}\left(\mathbb{R}^{3 d}\right)$. In this section, we will show that every algebraic tensor product of normed spaces can be seen as a Banach-Grassmann-like manifold.

\subsection{Tensor Spaces and the tensor product map}

All along this paper we consider a finite index set $D:=\{1,2, \ldots, d\}$ of 'spatial directions', with $d \geq 2$. Concerning the definition of the algebraic tensor space ${ }_{a} \bigotimes_{\alpha \in D} V_{\alpha}$ generated from vector spaces $V_{\alpha}(\alpha \in D)$, 
we refer to Greub [14]. As underlying field we choose $\mathbb{R}$, but the results hold also for $\mathbb{C}$. The suffix ' $a$ ' in ${ }_{a} \bigotimes_{\alpha \in D} V_{\alpha}$ refers to the 'algebraic' nature. By definition, all elements of

$$
\mathbf{V}_{D}:={ }_{a} \bigotimes_{\alpha \in D} V_{\alpha}
$$

are finite linear combinations of elementary tensors $\mathbf{v}=\bigotimes_{\alpha \in D} v_{\alpha}\left(v_{\alpha} \in V_{\alpha}\right)$. In the sequel, the index sets $D \backslash\{\alpha\}$ will appear. Here, we use the abbreviations

$$
\mathbf{V}_{[\alpha]}:={ }_{a} \bigotimes_{\beta \neq \alpha} V_{\beta}, \quad \text { where } \bigotimes_{\beta \neq \alpha} \text { means } \bigotimes_{\beta \in D \backslash\{\alpha\}} .
$$

Similarly, elementary tensors $\bigotimes_{\beta \neq \alpha} v_{\beta}$ are denoted by $\mathbf{v}_{[\alpha]}$. We notice that there exists a linear isomorphism $\Phi_{\alpha}: \mathbf{V}_{D} \longrightarrow V_{\alpha} \otimes \otimes \mathbf{V}_{[\alpha]}$ for each $\alpha \in D$, and in order to simplify notation we will identify along the text a tensor $\mathbf{v} \in \mathbf{V}_{D}$ with $\Phi_{\alpha}(\mathbf{v}) \in V_{\alpha} a_{a} \otimes \mathbf{V}_{[\alpha]}$. This allows us to write $\mathbf{v} \in \mathbf{V}_{D}$ as well as $\mathbf{v} \in V_{\alpha} a_{a} \mathbf{V}_{[\alpha]}$ for $\alpha \in D$. Moreover, by the universal property of the tensor product, there exists a unique multilinear map, also denoted by $\otimes$

$$
\bigotimes: \underset{\alpha \in D}{\chi} V_{\alpha} \longrightarrow{ }_{a} \bigotimes_{\alpha \in D} V_{\alpha}
$$

defined by $\otimes\left(\left(v_{1}, \ldots, v_{d}\right)\right)=\bigotimes_{\alpha \in D} v_{\alpha}$ and such that for each multilinear map $M: \times_{\alpha \in D} V_{\alpha} \longrightarrow Z$, where $Z$ is a given vector space, there exists a unique map $\widehat{M}: \mathbf{V}_{D} \rightarrow Z$ such that $M=\widehat{M} \circ \otimes$. The following notations, definitions and results will be useful.

Let $\left(V_{\alpha},\|\cdot\|_{\alpha}\right)$ be normed spaces for $\alpha \in D$ and assume that $\|\cdot\|$ is a norm on the tensor space $\mathbf{V}_{D}={ }_{a} \bigotimes_{\alpha \in D} V_{\alpha}$. Then consider the tensor product map

$$
\bigotimes:\left(\underset{\alpha \in D}{X} V_{\alpha},\|\cdot\|_{\times}\right) \rightarrow\left({ }_{a} \bigotimes_{\alpha \in D} V_{\alpha},\|\cdot\|\right)
$$

where the product space $\times_{\alpha \in D} V_{\alpha}$ is equipped with the product topology induced by the maximum norm $\left\|\left(v_{1}, \ldots, v_{d}\right)\right\|_{\times}=\max _{\alpha \in D}\left\|v_{\alpha}\right\|_{\alpha}$. Next, we discuss the conditions for having the Fréchet differentiability of the tensor product map (3.1). The next result is a consequence of Proposition 2.1.

Proposition 3.1 Let $\left(V_{\alpha},\|\cdot\|_{\alpha}\right)$ be normed spaces for $\alpha \in D$. Assume that $\|\cdot\|$ is a norm on the tensor space $\mathbf{V}_{D}={ }_{a} \bigotimes_{\alpha \in D} V_{\alpha}$ such that the tensor product map (3.1) is continuous. Then it is also $\mathcal{C}^{\infty}$-Fréchet differentiable and its differential is given by

$$
D\left(\bigotimes\left(v_{1}, \ldots, v_{d}\right)\right)\left(w_{1}, \ldots, w_{d}\right)=\sum_{\alpha \in D} v_{1} \otimes \ldots \otimes v_{\alpha-1} \otimes w_{\alpha} \otimes v_{\alpha+1} \otimes \cdots v_{d} .
$$

Now, we recall the definition of some topological tensor spaces and we will give some examples.

Definition 3.2 We say that $\mathbf{V}_{D_{\|\cdot\|}}$ is a Banach tensor space if there exists an algebraic tensor space $\mathbf{V}_{D}$ and a norm $\|\cdot\|$ on $\mathbf{V}_{D}$ such that $\mathbf{V}_{D_{\|\cdot\|}}$ is the completion of $\mathbf{V}_{D}$ with respect to the norm $\|\cdot\|$, i.e.

$$
\mathbf{V}_{D_{\|\cdot\|}:=\|\cdot\|} \bigotimes_{\alpha \in D} V_{\alpha}=\bar{a}_{{ }_{a} \bigotimes_{\alpha \in D} V_{\alpha}} \cdot \cdot \|
$$

If $\mathbf{V}_{D_{\|\cdot\|}}$ is a Hilbert space, we say that $\mathbf{V}_{D_{\|\cdot\|}}$ is a Hilbert tensor space.

Next, we give some examples of Banach and Hilbert tensor spaces.

Example 3.3 For $I_{\alpha} \subset \mathbb{R}(\alpha \in D)$ and $1 \leq p<\infty$, the Sobolev space $H^{N, p}\left(I_{\alpha}\right)$ consists of all univariate functions $f$ in $L^{p}\left(I_{\alpha}\right)$ with bounded norm ${ }^{2}$

$$
\|f\|_{N, p ; I_{\alpha}}:=\left(\sum_{n=0}^{N} \int_{I_{\alpha}}\left|\partial^{n} f\right|^{p} \mathrm{~d} x\right)^{1 / p},
$$

\footnotetext{
${ }^{2}$ It suffices to have in (3.2) the terms $n=0$ and $n=N$. The derivatives are to be understood as weak derivatives.
} 
whereas the space $H^{N, p}(\mathbf{I})$ of d-variate functions on $\mathbf{I}=I_{1} \times I_{2} \times \ldots \times I_{d} \subset \mathbb{R}^{d}$ consists of all functions $f$ in $L^{p}(\mathbf{I})$ with bounded norm

$$
\|f\|_{N, p}:=\left(\sum_{0 \leq|\mathbf{n}| \leq N} \int_{\mathbf{I}}\left|\partial^{\mathbf{n}} f\right|^{p} \mathrm{~d} \mathbf{x}\right)^{1 / p}
$$

with $\mathbf{n} \in \mathbb{N}_{0}^{d}$ being a multi-index of length $|\mathbf{n}|:=\sum_{\alpha \in D} n_{\alpha}$. For $p>1$ it is well known that $H^{N, p}\left(I_{\alpha}\right)$ and $H^{N, p}(\mathbf{I})$ are reflexive and separable Banach spaces. Moreover, for $p=2$, the Sobolev spaces $H^{N}\left(I_{\alpha}\right):=$ $H^{N, 2}\left(I_{\alpha}\right)$ and $H^{N}(\mathbf{I}):=H^{N, 2}(\mathbf{I})$ are Hilbert spaces. As a first example,

$$
H^{N, p}(\mathbf{I})=\|\cdot\|_{N, p} \bigotimes_{\alpha \in D} H^{N, p}\left(I_{\alpha}\right)
$$

is a Banach tensor space. Examples of Hilbert tensor spaces are

$$
L^{2}(\mathbf{I})=\|\cdot\|_{0,2} \bigotimes_{\alpha \in D} L^{2}\left(I_{\alpha}\right) \quad \text { and } \quad H^{N}(\mathbf{I})=\|\cdot\|_{N, 2} \bigotimes_{\alpha \in D} H^{N}\left(I_{\alpha}\right) \text { for } N \in \mathbb{N} \text {. }
$$

The next result is a consequence of Corollary 2.2

Proposition 3.4 Let $\left(V_{\alpha},\|\cdot\|_{\alpha}\right)$ be complex Banach spaces for $\alpha \in D$. Assume that $\|\cdot\|$ is a norm on the complex tensor space $\mathbf{V}_{D}={ }_{a} \bigotimes_{\alpha \in D} V_{\alpha}$ such that the tensor product map (3.1) is continuous. Let $\mathfrak{i}: \mathbf{V}_{D} \rightarrow\|\cdot\| \bigotimes_{\alpha \in D} V_{\alpha}$ be the standard inclusion map, i.e. $\mathfrak{i}(\mathbf{v})=\mathbf{v}$. Then

$$
(\mathfrak{i} \circ \bigotimes): \underset{\alpha \in D}{X}\left(V_{\alpha},\|\cdot\|_{\alpha}\right) \longrightarrow\left(\|\cdot\| \bigotimes_{\alpha \in D} V_{\alpha},\|\cdot\|\right)
$$

is an analytic map between complex Banach spaces.

For vector spaces $V_{\alpha}$ and $W_{\alpha}$ over $\mathbb{R}$, let linear mappings $A_{\alpha}: V_{\alpha} \rightarrow W_{\alpha}(\alpha \in D)$ be given. Then the definition of the elementary tensor

$$
\mathbf{A}=\bigotimes_{\alpha \in D} A_{\alpha}: \mathbf{V}_{D}=\bigotimes_{a} V_{\alpha \in D} \longrightarrow \mathbf{W}_{D}={ }_{a} \bigotimes_{\alpha \in D} W_{\alpha}
$$

is given by

$$
\mathbf{A}\left(\bigotimes_{\alpha \in D} v_{\alpha}\right):=\bigotimes_{\alpha \in D}\left(A_{\alpha} v_{\alpha}\right)
$$

Note that (3.3) uniquely defines the linear mapping $\mathbf{A}: \mathbf{V}_{D} \rightarrow \mathbf{W}_{D}$. We recall that $L(V, W)$ is the space of linear maps from $V$ into $W$, while $V^{\prime}=L(V, \mathbb{R})$ is the algebraic dual of $V$. For metric spaces, $\mathcal{L}(V, W)$ denotes the continuous linear maps, while $V^{*}=\mathcal{L}(V, \mathbb{R})$ is the topological dual of $V$.

Proposition 3.5 Let $\left(V_{\alpha},\|\cdot\|_{\alpha}\right)$ be normed spaces for $\alpha \in D$ and assume that $\|\cdot\|$ is a norm on the tensor space $\mathbf{V}_{D}={ }_{a} \bigotimes_{\alpha \in D} V_{\alpha}$ such that the tensor product map (3.1) is continuous. Let $U_{\alpha}$ be a finite-dimensional subspace of $V_{\alpha}$ for $\alpha \in D$. Then

$$
\bigotimes_{\alpha \in D} \mathcal{L}\left(U_{\alpha}, V_{\alpha}\right)=\mathcal{L}\left({ }_{a} \bigotimes_{\alpha \in D} U_{\alpha}, \mathbf{V}_{D}\right)
$$

and the tensor product map

$$
\bigotimes: \underset{\alpha \in D}{\chi} \mathcal{L}\left(U_{\alpha}, V_{\alpha}\right) \rightarrow \mathcal{L}\left(\bigotimes_{a} U_{\alpha \in D}, \mathbf{V}_{D}\right), \quad\left(A_{\alpha}\right)_{\alpha \in D} \mapsto \mathbf{A}:=\bigotimes_{\alpha \in D} A_{\alpha}
$$

is continuous and hence $\mathcal{C}^{\infty}$-Fréchet differentiable. 
Proof. Recall that $L(U, X)=\mathcal{L}(U, X)$ holds for every finite-dimensional subspace $U$ of a normed space $X$. Then (3.4) follows from Proposition 3.49 of [19]. To prove the second statement we need to show that the tensor product map (3.5) is bounded, that is,

$$
\|\bigotimes\|=\sup \left\{\left\|\bigotimes_{\alpha \in D} A_{\alpha}\right\|_{\mathbf{v}_{D} \leftarrow{ }_{a}} \bigotimes_{\alpha \in D} U_{\alpha}:\left\|A_{\alpha}\right\|_{V_{\alpha} \leftarrow U_{\alpha}} \leq 1 \text { for } 1 \leq \alpha \leq d\right\}<\infty .
$$

For $\mathbf{A}=\bigotimes_{\alpha \in D} A_{\alpha}$,

$$
\left\|\mathbf{A}\left(\otimes_{\alpha \in D} u_{\alpha}\right)\right\|=\left\|\otimes_{\alpha \in D} A_{\alpha}\left(u_{\alpha}\right)\right\| \leq C \prod_{\alpha \in D}\left\|A_{\alpha}\left(u_{\alpha}\right)\right\|_{\alpha} \leq C \prod_{\alpha \in D}\left\|A_{\alpha}\right\|_{V_{\alpha} \leftarrow U_{\alpha}}\left\|u_{\alpha}\right\|_{\alpha}
$$

holds by the continuity of the tensor product map (3.1). Therefore,

$$
\|\mathbf{A}\|_{\mathbf{v}_{D} \leftarrow a} \otimes_{\alpha \in D} U_{\alpha}=\sup \left\{\|\mathbf{A}(\mathbf{u})\|: \mathbf{u} \in \bigotimes_{\alpha \in D} U_{\alpha},\|\mathbf{u}\| \leq 1\right\} \leq C^{\prime} \prod_{\alpha \in D}\left\|A_{\alpha}\right\|_{V_{\alpha} \leftarrow U_{\alpha}},
$$

for some constant $C^{\prime}$ depending on the dimension of the spaces $U_{\alpha}, \alpha \in D$, and (3.6) follows. From Proposition 2.1 the second statement holds.

\subsection{The set of tensors in Tucker format with fixed rank}

Before introducing the manifold of tensors in Tucker format with fixed rank in a Banach space framework, we need to define the minimal subspace of a tensor in an algebraic tensor space. The following statement summarises the results given in Section 2.2 in [11.

Proposition 3.6 Given a finite index set $D=\{1,2, \ldots, d\}$, let $V_{\alpha}$ be a vector space for each $\alpha \in D$ and let $\mathbf{v} \in{ }_{a} \bigotimes_{\alpha \in D} V_{\alpha}$. Then for each $\alpha \in D$ there exists a unique subspace $U_{\alpha}^{\min }(\mathbf{v})$ with $\operatorname{dim} U_{\alpha}^{\min }(\mathbf{v})=r_{\alpha}$ for some $r_{\alpha}<\infty$, and such that the following statements hold.

(a) If $\mathbf{v} \in{ }_{a} \bigotimes_{\alpha \in D} U_{\alpha}$ then $U_{\alpha}^{\min }(\mathbf{v}) \subset U_{\alpha}(\alpha \in D)$, while $\mathbf{v} \in{ }_{a} \bigotimes_{\alpha \in D} U_{\alpha}^{\min }(\mathbf{v})$.

(b) For each $\alpha \in D$ there exists a unique subspace $U_{D \backslash\{\alpha\}}^{\min }(\mathbf{v}) \subset \mathbf{V}_{[\alpha]}$ such that $\mathbf{v} \in U_{\alpha}^{\min }(\mathbf{v}) \otimes_{a} U_{D \backslash\{\alpha\}}^{\min }(\mathbf{v})$ and $\operatorname{dim} U_{D \backslash\{\alpha\}}^{\min }(\mathbf{v})=r_{\alpha}$.

For a tensor $\mathbf{v} \in \mathbf{V}_{D}={ }_{a} \bigotimes_{\alpha \in D} V_{\alpha}$ the linear subspaces $U_{\alpha}^{\min }(\mathbf{v})(\alpha \in D)$ are called minimal subspaces and $r_{\alpha}=\operatorname{dim} U_{\alpha}^{\min }(\mathbf{v})$ is called the $\alpha$-rank of $\mathbf{v}$.

Let $\mathbb{Z}_{+}$be the set of non-negative integers. We will say that $\mathfrak{r}=\left(r_{1}, \ldots, r_{d}\right) \in \mathbb{Z}_{+}^{d}$ is an admissible rank for $\mathbf{V}_{D}:={ }_{a} \bigotimes_{\alpha \in D} V_{\alpha}$ if and only if there exists $\mathbf{v} \in \mathbf{V}_{D}$ such that $r_{\alpha}=\operatorname{dim} U_{\alpha}^{\min }(\mathbf{v})$ for $\alpha \in D$. We will denote the set of all admissible ranks of a tensor space $\mathbf{V}_{D}$ by $\mathcal{A D}\left(\mathbf{V}_{D}\right)$, and hence

$$
\mathcal{A D}\left(\mathbf{V}_{D}\right)=\left\{\left(\operatorname{dim} U_{\alpha}^{\min }(\mathbf{v})\right)_{\alpha \in D} \in \mathbb{Z}_{+}^{d}: \mathbf{v} \in \mathbf{V}_{D}\right\}
$$

It is not difficult to see that $\mathbf{0}=(0, \ldots, 0) \in \mathcal{A D}\left(\mathbf{V}_{D}\right)$ and $\mathbf{1}=(1, \ldots, 1) \in \mathcal{A D}\left(\mathbf{V}_{D}\right)$ if and only if $\operatorname{dim} V_{\alpha} \geq 1$ for all $\alpha \in D$.

Now, we define in an algebraic tensor space $\mathbf{V}_{D}={ }_{a} \bigotimes_{\alpha \in D} V_{\alpha}$ the set of tensors in Tucker format with fixed rank $\mathfrak{r}=\left(r_{1}, \ldots, r_{d}\right) \in \mathcal{A D}\left(\mathbf{V}_{D}\right)$ by

$$
\mathfrak{M}_{\mathfrak{r}}\left(\mathbf{V}_{D}\right):=\left\{\mathbf{v} \in \mathbf{V}_{D}: \operatorname{dim} U_{\alpha}^{\min }(\mathbf{v})=r_{\alpha}, \alpha \in D\right\}
$$

Then

$$
\mathbf{V}_{D}=\bigcup_{\mathfrak{r} \in \mathcal{A D}\left(\mathbf{V}_{D}\right)} \mathfrak{M}_{\mathfrak{r}}\left(\mathbf{V}_{D}\right)
$$

Before introducing the representation of a tensor with a fixed rank $\mathfrak{r}$ we need to define the set of coefficients of that tensors. To this end, we recall the definition of the 'matricisation' (or 'unfolding') of a tensor in a finite-dimensional setting. 
Definition 3.7 For a finite index set $D=\{1,2, \ldots, d\}, d \geq 2$, and each $\mu \in D$ the map $\mathcal{M}_{\mu}$ is defined as the isomorphism

$$
\begin{aligned}
\mathcal{M}_{\mu}: & \mathbb{R}^{\times_{\beta \in D} r_{\beta}} \rightarrow \mathbb{R}^{r_{\mu} \times\left(\prod_{\delta \in D \backslash\{\mu\}} r_{\delta}\right)} \\
& C_{\left(i_{\beta}\right)_{\beta \in D}} \mapsto C_{i_{\mu},\left(i_{\delta}\right)_{\delta \in D \backslash\{\mu\}}}
\end{aligned}
$$

It allows us to introduce the following definition.

Definition 3.8 For a finite index set $D=\{1,2, \ldots, d\}, d \geq 2$, let $C^{(D)} \in \mathbb{R}^{\times_{\mu \in D} r_{\mu}}$. We say that $C^{(D)} \in$ $\mathbb{R}_{*}^{\times_{\mu \in D} r_{\mu}}$ if and only if $\operatorname{rank} \mathcal{M}_{\mu}\left(C^{(D)}\right)=r_{\mu}$, where $\mathcal{M}_{\mu}\left(C^{(D)}\right) \in \mathbb{R}^{r_{\mu} \times\left(\Pi_{\beta \in D \backslash\{\mu\}}{ }^{r_{\beta}}\right)}$, for each $\mu \in D$.

Remark 3.9 We have that $C^{(D)} \in \mathbb{R}_{*}^{\times_{\mu \in D} r_{\mu}}$ if and only if $\mathcal{M}_{\mu}\left(C^{(D)}\right) \mathcal{M}_{\mu}\left(C^{(D)}\right)^{T} \in \operatorname{GL}\left(\mathbb{R}^{r_{\mu}}\right)$ for $\mu \in D$. Since the determinant is a continuous function, $\mathbb{R}_{*}^{\times_{\mu \in D} r_{\mu}}$ is an open set in $\mathbb{R}^{\times_{\mu \in D} r_{\mu}}$ and hence a finite-

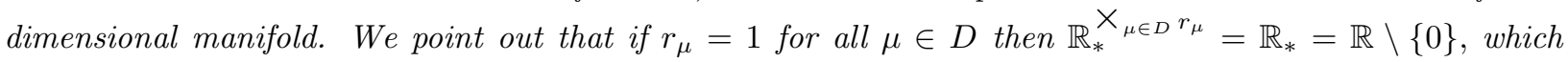
coincides with the Lie group $\mathrm{GL}(\mathbb{R})$.

In the next lemma we give a characterisation of the representation of tensors in $\mathfrak{M}_{\mathfrak{r}}\left(\mathbf{V}_{D}\right)$.

Lemma 3.10 Let $\mathbf{V}_{D}={ }_{a} \bigotimes_{\alpha \in D} V_{\alpha}$ be an algebraic tensor space. Then the following statements are equivalent.

(a) $\mathbf{v} \in \mathfrak{M}_{\mathfrak{r}}\left(\mathbf{V}_{D}\right)$

(b) For each $\alpha \in D$ there exists a set $\mathcal{B}_{\alpha}=\left\{u_{i_{\alpha}}^{(\alpha)}: 1 \leq i_{\alpha} \leq r_{\alpha}\right\}$ of linearly independent vectors and a unique $C^{(D)} \in \mathbb{R}_{*}^{\times_{\alpha \in D} r_{\alpha}}$, once $\mathcal{B}_{\alpha}$ is fixed $(\alpha \in D)$, such that

$$
\mathbf{v}=\sum_{\substack{1 \leq i_{\alpha} \leq r_{\alpha} \\ \alpha \in D}} C_{\left(i_{\alpha}\right)_{\alpha \in D}}^{(D)} \bigotimes_{\alpha \in D} u_{i_{\alpha}}^{(\alpha)}
$$

(c) For each $\alpha \in D$ there exist linearly independent vectors $\left\{u_{i_{\alpha}}^{(\alpha)}: 1 \leq i_{\alpha} \leq r_{\alpha}\right\}$ in $V_{\alpha}$ and linearly independent vectors $\left\{\mathbf{U}_{i_{\alpha}}^{(\alpha)}: 1 \leq i_{\alpha} \leq r_{\alpha}\right\}$ in $\mathbf{V}_{[\alpha]}={ }_{a} \bigotimes_{\beta \in D \backslash\{\alpha\}} V_{\beta}$ such that

$$
\mathbf{v}=\sum_{1 \leq i_{\alpha} \leq r_{\alpha}} u_{i_{\alpha}}^{(\alpha)} \otimes \mathbf{U}_{i_{\alpha}}^{(\alpha)}
$$

Furthermore, if (3.7) holds, then

$$
\mathbf{U}_{i_{\alpha}}^{(\alpha)}=\sum_{\substack{1 \leq i_{\beta} \leq r_{\beta} \\ \beta \in D \backslash\{\alpha\}}} C_{i_{\alpha},\left(i_{\beta}\right)_{\beta \in D \backslash\{\alpha\}}}^{(D)} \bigotimes_{\beta \in D} u_{i_{\beta}}^{(\beta)}
$$

for $1 \leq i_{\alpha} \leq r_{\alpha}$ and $\alpha \in D$.

Proof. First, we prove that (a) and (c) are equivalent. If (a) holds, then from Proposition 3.6 (b) we know that

$$
\mathbf{v} \in U_{\alpha}^{\min }(\mathbf{v}) \otimes_{a} U_{D \backslash\{\alpha\}}^{\min }(\mathbf{v})
$$

where $\operatorname{dim} U_{\alpha}^{\min }(\mathbf{v})=\operatorname{dim} U_{D \backslash\{\alpha\}}^{\min }(\mathbf{v})=r_{\alpha}$ for each $\alpha \in D$. Then there exists linearly independent vectors $\left\{u_{i_{\alpha}}^{(\alpha)}: 1 \leq i_{\alpha} \leq r_{\alpha}\right\}$ in $V_{\alpha}$ and linearly independent vectors $\left\{\mathbf{U}_{i_{\alpha}}^{(\alpha)}: 1 \leq i_{\alpha} \leq r_{\alpha}\right\}$ in $\mathbf{V}_{[\alpha]}={ }_{a} \bigotimes_{\beta \in D \backslash\{\alpha\}} V_{\beta}$ such that (3.8) holds and hence (c) is true. Conversely, if (c) holds then clearly $\operatorname{dim} U_{\alpha}^{\min }(\mathbf{v})=r_{\alpha}$ for each $\alpha \in D$, and hence (a) is also true.

Now, we prove that (b) and (c) are equivalent. Clearly (b) implies (c). To prove that (c) implies (b) assume that (c) holds. By the definition of minimal subspace we have that

$$
U_{\alpha}^{\min }(\mathbf{v})=\operatorname{span}\left\{u_{i_{\alpha}}^{(\alpha)}: 1 \leq i_{\alpha} \leq r_{\alpha}\right\}
$$


for each $\alpha \in D$. Since $\mathbf{v} \in{ }_{a} \bigotimes_{\alpha \in D} U_{\alpha}^{\min }(\mathbf{v})$ there exists $C^{(D)} \in \mathbb{R}^{\times_{\alpha \in D} r_{\alpha}}$ such that (3.7) holds. To conclude the proof we only need to show that $C^{(D)} \in \mathbb{R}_{*}^{\times_{\alpha \in D} r_{\alpha}}$. To this end observe that (3.9) must hold for $1 \leq i_{\alpha} \leq r_{\alpha}$ and each $\alpha \in D$, and hence rank $\mathcal{M}_{\alpha}\left(C^{(D)}\right)=r_{\alpha}$ for each $\alpha \in D$. In consequence, $C^{(D)} \in \mathbb{R}_{*}^{\times_{\alpha \in D} r_{\alpha}}$ and (b) is true.

Remark 3.11 From the proof of Lemma 3.10 we have that $U_{\alpha}^{\min }(\mathbf{v})=\operatorname{span}\left\{u_{i_{\alpha}}^{(\alpha)}: 1 \leq i_{\alpha} \leq r_{\alpha}\right\}$ and $U_{D \backslash\{\alpha\}}^{\min }(\mathbf{v})=\operatorname{span}\left\{\mathbf{U}_{i_{\alpha}}^{(\alpha)}: 1 \leq i_{\alpha} \leq r_{\alpha}\right\}$ for each $\alpha \in D$. Furthermore, for

$$
\mathbf{v}=\sum_{\substack{1 \leq i_{\alpha} \leq r_{\alpha} \\ \alpha \in D}} C_{\left(i_{\alpha}\right)_{\alpha \in D}}^{(D)} \bigotimes_{\alpha \in D} u_{i_{\alpha}}^{(\alpha)} \in \mathfrak{M}_{\mathfrak{r}}\left(\mathbf{V}_{D}\right)
$$

there exists a natural diffeomorphism

$$
\mathfrak{M}_{\mathfrak{r}}\left({ }_{a} \bigotimes_{\alpha \in D} U_{\alpha}^{\min }(\mathbf{v})\right) \rightarrow \mathbb{R}_{*}^{\times_{\alpha \in D} r_{\alpha}}, \quad \sum_{\substack{1 \leq i_{\alpha} \leq r_{\alpha} \\ \alpha \in D}} E_{\left(i_{\alpha}\right)_{\alpha \in D}}^{(D)} \bigotimes_{\alpha \in D} u_{i_{\alpha}}^{(\alpha)} \mapsto E^{(D)} .
$$

Thus we will identify each $u \in \mathfrak{M}_{\mathfrak{r}}\left({ }_{a} \bigotimes_{\alpha \in D} U_{\alpha}^{\min }(\mathbf{v})\right)$ with an element $E^{(D)} \in \mathbb{R}_{*}{ }_{\alpha \in D} r_{\alpha}$, once a basis $\left\{u_{i_{\alpha}}^{(\alpha)}: 1 \leq i_{\alpha} \leq r_{\alpha}\right\}$ of $U_{\alpha}^{\min }(\mathbf{v})$ is fixed for each $\alpha \in D$, by means of the equality

$$
\mathbf{u}=\mathbf{u}\left(E^{(D)}\right)=\sum_{\substack{1 \leq i_{\alpha} \leq r_{\alpha} \\ \alpha \in D}} E_{\left(i_{\alpha}\right)_{\alpha \in D}}^{D} \bigotimes_{\alpha \in D} u_{i_{\alpha}}^{(\alpha)}
$$

\subsection{The manifold of tensors in Tucker format with fixed rank}

Assume that $\left(V_{\alpha},\|\cdot\|_{\alpha}\right)$ is a normed space and denote by $V_{\alpha_{\|\cdot\| \alpha}}$ the Banach space obtained by the completion of $V_{\alpha}$ for each $\alpha \in D$. Moreover, we also assume that $\|\cdot\|_{D}$ is a norm on the tensor space $\mathbf{V}_{D}={ }_{a} \otimes_{\alpha \in D} V_{\alpha}$ such that the tensor product map (3.1) is continuous and hence, by Proposition 3.1 it is also $\mathcal{C}^{\infty}$-Fréchet differentiable.

Now, we proceed to provide a geometric structure for the set $\mathfrak{M}_{\mathfrak{r}}\left(\mathbf{V}_{D}\right)$. By Proposition 3.6 and Example 2.24 we have that for each $\mathbf{v} \in \mathbf{V}_{D}$ the set $U_{\alpha}^{\min }(\mathbf{v}) \in \mathbb{G}_{r_{\alpha}}\left(V_{\alpha}\right)$ for some $r_{\alpha}<\infty$ and $\alpha \in D$. Since $\mathbf{V}_{D}=\bigcup_{\mathfrak{r} \in \mathcal{A} \mathcal{D}\left(\mathbf{V}_{D}\right)} \mathfrak{M}_{\mathfrak{r}}\left(\mathbf{V}_{D}\right)$, thanks to Proposition 3.6 we can define a surjective map from a tensor space to an analytic Banach manifold:

$$
\varrho: \mathbf{V}_{D} \longrightarrow \bigcup_{\left(r_{1}, \ldots, r_{d}\right) \in \mathcal{A} \mathcal{D}\left(\mathbf{V}_{D}\right)}\left(\underset{\alpha \in D}{X} \mathbb{G}_{r_{\alpha}}\left(V_{\alpha}\right)\right), \quad \mathbf{v} \mapsto\left(U_{\alpha}^{\min }(\mathbf{v})\right)_{\alpha \in D} .
$$

It allows us to consider for a fixed $\mathfrak{r} \in \mathcal{A D}\left(\mathbf{V}_{D}\right), \mathfrak{r} \neq \mathbf{0}$, the restricted map

$$
\varrho_{\mathfrak{r}}=\left.\varrho\right|_{\mathfrak{M}_{\mathfrak{r}}\left(\mathbf{V}_{D}\right)}: \mathfrak{M}_{\mathfrak{r}}\left(\mathbf{V}_{D}\right)=\varrho^{-1}\left(\underset{\alpha \in D}{X} \mathbb{G}_{r_{\alpha}}\left(V_{\alpha}\right)\right) \longrightarrow \underset{\alpha \in D}{X} \mathbb{G}_{r_{\alpha}}\left(V_{\alpha}\right), \quad \mathbf{v} \mapsto\left(U_{\alpha}^{\min }(\mathbf{v})\right)_{\alpha \in D},
$$

which is also surjective. For each $\alpha \in D$ the linear subspace $U_{\alpha}^{\min }(\mathbf{v}) \subset V_{\alpha} \subset V_{\alpha_{\|} \cdot \|_{\alpha}}$ belongs to the Banach manifold $\mathbb{G}_{r_{\alpha}}\left(V_{\alpha}\right)$ and hence there exists a closed subspace $W_{\alpha}^{\min }(\mathbf{v})$ such that $V_{\alpha_{\|\cdot\|_{\alpha}}}=U_{\alpha}^{\min }(\mathbf{v}) \oplus W_{\alpha}^{\min }(\mathbf{v})$ and a bijection (local chart)

$$
\Psi_{\mathbf{v}}^{(\alpha)}: \mathbb{G}\left(W_{\alpha}^{\min }(\mathbf{v}), \mathbf{V}_{\alpha_{\|\cdot\| \alpha}}\right) \rightarrow \mathcal{L}\left(U_{\alpha}^{\min }(\mathbf{v}), W_{\alpha}^{\min }(\mathbf{v})\right)
$$

given by

$$
\Psi_{\mathbf{v}}^{(\alpha)}\left(U_{\alpha}\right)=L_{\alpha}:=\left.P_{W_{\alpha}^{\min }(\mathbf{v}) \oplus U_{\alpha}^{\min }(\mathbf{v})}\right|_{U_{\alpha}} \circ\left(\left.P_{U_{\alpha}^{\min }(\mathbf{v}) \oplus W_{\alpha}^{\min }(\mathbf{v})}\right|_{U_{\alpha}}\right)^{-1} .
$$


Moreover, $U_{\alpha}=\left(\Psi_{\mathbf{v}}^{(\alpha)}\right)^{-1}\left(L_{\alpha}\right)=G\left(L_{\alpha}\right)=\operatorname{span}\left\{\left(i d_{\alpha}+L_{\alpha}\right)\left(u_{\alpha}\right): u_{\alpha} \in U_{\alpha}^{\min }(\mathbf{v})\right\}$. Clearly, the map

$$
\Psi_{\mathbf{v}}: \underset{\alpha \in D}{\chi} \mathbb{G}\left(W_{\alpha}^{\min }(\mathbf{v}), \mathbf{V}_{\alpha_{\|\cdot\| \alpha}}\right) \rightarrow \underset{\alpha \in D}{X} \mathcal{L}\left(U_{\alpha}^{\min }(\mathbf{v}), W_{\alpha}^{\min }(\mathbf{v})\right),
$$

defined as $\Psi_{\mathbf{v}}:=\chi_{\alpha \in D} \Psi_{\mathbf{v}}^{(\alpha)}$ is also bijective. Furthermore, it is a local chart for an element $\varrho_{\mathfrak{r}}(\mathbf{v})$ in the product manifold $X_{\alpha \in D} \mathbb{G}_{r_{\alpha}}\left(V_{\alpha}\right)$ such that $\Psi_{\mathbf{v}}\left(\varrho_{\mathfrak{r}}(\mathbf{v})\right)=\mathfrak{o}:=(0)_{k \in D}$.

Now, for each $\mathbf{v} \in \mathfrak{M}_{\mathfrak{r}}\left(\mathbf{V}_{D}\right)$ introduce the set

$$
\mathcal{U}(\mathbf{v}):=\varrho_{\mathfrak{r}}^{-1}\left(\underset{\alpha \in D}{X} \mathbb{G}\left(W_{\alpha}^{\min }(\mathbf{v}), V_{\alpha}\right)\right)=\left\{\mathbf{w} \in \mathfrak{M}_{\mathfrak{r}}\left(\mathbf{V}_{D}\right): U_{\alpha}^{\min }(\mathbf{w}) \in \mathbb{G}\left(W_{\alpha}^{\min }(\mathbf{v}), V_{\alpha}\right), \alpha \in D\right\}
$$

Recall that from Proposition 2.11 we can identify the linear space $\mathcal{L}\left(U_{\alpha}^{\min }(\mathbf{v}), W_{\alpha}^{\min }(\mathbf{v})\right)$ with a sub-algebra of $\mathcal{L}\left(V_{\alpha_{\|\cdot\|_{\alpha}}}, V_{\alpha_{\|\cdot\|_{\alpha}}}\right)$ for $\alpha \in D$. Then, from Example 2.20, the map

$$
\underset{\alpha \in D}{X} \mathcal{L}\left(U_{\alpha}^{\min }(\mathbf{v}), W_{\alpha}^{\min }(\mathbf{v})\right) \rightarrow \underset{\alpha \in D}{X} \mathcal{L}\left(U_{\alpha}^{\min }(\mathbf{v}), V_{\alpha}\right)
$$

between normed spaces given by

$$
\mathfrak{L}=\left(L_{\alpha}\right)_{\alpha \in D} \mapsto\left(\left.\left(i d_{\alpha}+L_{\alpha}\right)\right|_{U_{\alpha}^{\min }(\mathbf{v})}\right)_{\alpha \in D}=\left(\left.\exp \left(L_{\alpha}\right)\right|_{U_{\alpha}^{\min }(\mathbf{v})}\right)_{\alpha \in D}
$$

is clearly $\mathcal{C}^{\infty}$-Fréchet differentiable. Finally, from Proposition 3.5 the map

$$
\underset{\alpha \in D}{X} \mathcal{L}\left(U_{\alpha}^{\min }(\mathbf{v}), W_{\alpha}^{\min }(\mathbf{v})\right) \rightarrow \mathcal{L}\left({ }_{a} \bigotimes_{\alpha \in D} U_{\alpha}^{\min }(\mathbf{v}), \mathbf{V}_{D}\right)
$$

given by

$$
\mathfrak{L}=\left.\left(L_{\alpha}\right)_{\alpha \in D} \mapsto \bigotimes_{\alpha \in D} \exp \left(L_{\alpha}\right)\right|_{U_{\alpha}^{\min }(\mathbf{v})}
$$

is also $\mathcal{C}^{\infty}$-Fréchet differentiable.

Our next step is to characterise the representation of tensors that belong to $\mathcal{U}(\mathbf{v})$ by using the following lemma.

Lemma 3.12 Assume that $\left(V_{\alpha},\|\cdot\|_{\alpha}\right)$ is a normed space for each $\alpha \in D$ and that $\|\cdot\|_{D}$ is a norm on the tensor space $\mathbf{V}_{D}={ }_{a} \bigotimes_{\alpha \in D} V_{\alpha}$ such that the tensor product map (3.1) is continuous. For $\mathbf{v} \in \mathfrak{M}_{\mathfrak{r}}\left(\mathbf{V}_{D}\right)$ the following statements are equivalent.

(a) $\mathbf{w} \in \mathcal{U}(\mathbf{v})$.

(b) There exists a unique

$$
\left(\mathfrak{L}, \mathbf{u}\left(E^{(D)}\right)\right)=\left(\left(L_{\alpha}\right)_{\alpha \in D}, \mathbf{u}\left(E^{(D)}\right)\right) \in \underset{\alpha \in D}{X} \mathcal{L}\left(U_{\alpha}^{\min }(\mathbf{v}), W_{\alpha}^{\min }(\mathbf{v})\right) \times \mathfrak{M}_{\mathfrak{r}}\left({ }_{a} \bigotimes_{\alpha \in D} U_{\alpha}^{\min }(\mathbf{v})\right)
$$

such that

$$
\mathbf{w}=\left(\bigotimes_{\alpha \in D} \exp \left(L_{\alpha}\right)\right)\left(\mathbf{u}\left(E^{(D)}\right)\right)
$$

Proof. Assume that $\mathbf{w} \in \mathcal{U}(\mathbf{v})$. Then we have the following facts:

(i) From Lemma 3.10(b) there exist bases $\mathcal{B}_{\alpha}=\left\{u_{i_{\alpha}}^{(\alpha)}: 1 \leq i_{\alpha} \leq r_{\alpha}\right\}, \alpha \in D$, and a unique $C^{(D)} \in$ $\mathbb{R}_{*}^{\times_{\alpha \in D} r_{\alpha}}$, once the bases are fixed, such that $\mathbf{v}=\sum_{\substack{1 \leq i_{\alpha} \leq r_{\alpha} \\ \alpha \in D}} C_{\left(i_{\alpha}\right)_{\alpha \in D}}^{(D)} \bigotimes_{\alpha \in D} u_{i_{\alpha}}^{(\alpha)} \in \mathfrak{M}_{\mathfrak{r}}(\mathbf{v})$. From Remark 3.11. we know that $\mathcal{B}_{\alpha}$ is a basis of $U_{\alpha}^{\min }(\mathbf{v})$ for $\alpha \in D$. Now, we will consider that the bases $\mathcal{B}_{\alpha}, \alpha \in D$, are fixed. 
(ii) Since $U_{\alpha}^{\min }(\mathbf{w}) \in \mathbb{G}\left(W_{\alpha}^{\min }(\mathbf{v}), V_{\alpha_{\|\cdot\|_{\alpha}}}\right)$, for $\alpha \in D$, there exists a unique

$$
\mathfrak{L}=\left(L_{\alpha}\right)_{\alpha \in D} \in \underset{\alpha \in D}{X} \mathcal{L}\left(U_{\alpha}^{\min }(\mathbf{v}), W_{\alpha}^{\min }(\mathbf{v})\right)
$$

such that $\Psi_{\mathbf{v}}\left(\varrho_{\mathfrak{r}}(\mathbf{w})\right)=\mathfrak{L}$, that is, $U_{\alpha}^{\min }(\mathbf{w})=G\left(L_{\alpha}\right)=\operatorname{span}\left\{\left(i d_{\alpha}+L_{\alpha}\right)\left(u_{i_{\alpha}}^{(\alpha)}\right): 1 \leq i_{\alpha} \leq r_{\alpha}\right\}$ for all $\alpha \in D$. Then

$$
\left(U_{\alpha}^{\min }(\mathbf{w})\right)_{\alpha \in D}=\mathbf{\Psi}_{\mathbf{v}}^{-1}(\mathfrak{L})
$$

and we can construct from $\mathcal{B}_{\alpha}$, a basis of $U_{\alpha}^{\min }(\mathbf{w})$. In particular we have $\Psi_{\mathbf{v}}\left(\varrho_{\mathfrak{r}}(\mathbf{v})\right)=(0)_{\alpha \in D}$.

(iii) Now by Lemma 3.10 (b), since a basis of $U_{\alpha}^{\min }(\mathbf{w})=G\left(L_{\alpha}\right)=\operatorname{span}\left\{\left(i d_{\alpha}+L_{\alpha}\right)\left(u_{i_{\alpha}}^{(\alpha)}\right): 1 \leq i_{\alpha} \leq r_{\alpha}\right\}$ for $\alpha \in D$ is fixed, there exists a unique $E^{(D)} \in \mathbb{R}_{*}^{\mathfrak{r}}$ such that

$$
\mathbf{w}=\sum_{\substack{1 \leq i_{\alpha} \leq r_{\alpha} \\ \alpha \in D}} E_{\left(i_{\alpha}\right)_{\alpha \in D}}^{(D)} \bigotimes_{\alpha \in D}\left(i d_{\alpha}+L_{\alpha}\right)\left(u_{i_{\alpha}}^{(\alpha)}\right)=\bigotimes_{\alpha \in D}\left(i d_{\alpha}+L_{\alpha}\right)\left(\mathbf{u}\left(E^{(D)}\right)\right)
$$

where

$$
\mathbf{u}\left(E^{(D)}\right):=\sum_{\substack{1 \leq i_{\alpha} \leq r_{\alpha} \\ \alpha \in D}} E_{\left(i_{\alpha}\right)_{\alpha \in D}}^{(D)} \bigotimes_{\alpha \in D} u_{i_{\alpha}}^{(\alpha)} \in \mathfrak{M}_{\mathfrak{r}}\left(\bigotimes_{a \in D} U_{\alpha}^{\min }(\mathbf{v})\right)=\mathbb{R}_{*}^{\times_{\alpha \in D} r_{\alpha}}
$$

It follows (b). From what was said above, (b) clearly implies (a).

Remark 3.13 We can interpret Lemma 3.12 as follows. $\mathbf{w} \in \mathcal{U}(\mathbf{v})$ holds if and only if

$$
\mathbf{w} \in\left(\bigotimes_{\alpha \in D} \exp \left(L_{\alpha}\right)\right)\left(\mathfrak{M}_{\mathfrak{r}}\left({ }_{a} \bigotimes_{\alpha \in D} U_{\alpha}^{\min }(\mathbf{v})\right)\right)
$$

for some $\mathfrak{L}=\left(L_{\alpha}\right)_{\alpha \in D} \in X_{\alpha \in D} \mathcal{L}\left(U_{\alpha}^{\min }(\mathbf{v}), W_{\alpha}^{\min }(\mathbf{v})\right)$. In consequence, each neighbourhood of $\mathbf{v}$ in $\mathfrak{M}_{\mathfrak{r}}\left(\mathbf{V}_{D}\right)$ can be written as

$$
\mathcal{U}(\mathbf{v})=\bigcup_{\mathfrak{L} \in X_{\alpha \in D} \mathcal{L}\left(U_{\alpha}^{\min }(\mathbf{v}), W_{\alpha}^{\min }(\mathbf{v})\right)}\left(\bigotimes_{\alpha \in D} \exp \left(L_{\alpha}\right)\right)\left(\mathfrak{M}_{\mathfrak{r}}\left({ }_{a} \bigotimes_{\alpha \in D} U_{\alpha}^{\min }(\mathbf{v})\right)\right),
$$

that is, a union of manifolds (each of them diffeomeorphic to $\mathbb{R}_{*}^{\times_{\alpha \in D} r_{\alpha}}$ ) indexed by a Banach manifold.

Now, also by using Lemma 3.12 we construct an explicit manifold structure for $\mathfrak{M}_{\mathfrak{r}}\left(\mathbf{V}_{D}\right)$. Indeed, Lemma 3.12 allows us to define for each $\mathbf{v} \in \mathfrak{M}_{\mathfrak{r}}(\mathbf{v})$, once a basis of $U_{\alpha}^{\min }(\mathbf{v})$ for each $\alpha \in D$ is fixed, a bijective map

$$
\xi_{\mathbf{v}}: \mathcal{U}(\mathbf{v}) \rightarrow\left(\underset{\alpha \in D}{X} \mathcal{L}\left(U_{\alpha}^{\min }(\mathbf{v}), W_{\alpha}^{\min }(\mathbf{v})\right)\right) \times \mathbb{R}_{*}^{\times_{\alpha \in D} r_{\alpha}}
$$

by

$$
\xi_{\mathbf{v}}\left(\left(\bigotimes_{\alpha \in D} \exp \left(L_{\alpha}\right)\right)\left(\mathbf{u}\left(C^{(D)}\right)\right)\right):=\left(\mathfrak{L}, C^{(D)}\right),
$$

where $\mathfrak{L}:=\left(L_{\alpha}\right)_{\alpha \in D}$. Clearly, $\xi_{\mathbf{v}}$ is a bijective map and hence $\mathcal{U}(\mathbf{v})$ can be identified with the Banach manifold

$$
\left(\underset{\alpha \in D}{X} \mathbb{G}\left(W_{\alpha}^{\min }(\mathbf{v}), V_{\alpha}\right)\right) \times \mathbb{R}_{*}^{\times_{\alpha \in D} r_{\alpha}},
$$


which is modelled on the Banach space

$$
\left(\underset{\alpha \in D}{\times} \mathcal{L}\left(U_{\alpha}^{\min }(\mathbf{v}), W_{\alpha}^{\min }(\mathbf{v})\right)\right) \times \mathbb{R}^{\times_{\alpha \in D} r_{\alpha}}
$$

The next lemma allows us to prove that $\left\{\left(\mathcal{U}(\mathbf{v}), \xi_{\mathbf{v}}\right)\right\}_{\mathbf{v} \in \mathfrak{M}_{\mathbf{r}}\left(\mathbf{V}_{D}\right)}$ is a local chart system for the set of tensors in Tucker format with fixed rank $\mathfrak{r}$.

Lemma 3.14 Assume that $\left(V_{\alpha},\|\cdot\|_{\alpha}\right)$ is a normed space for each $\alpha \in D$ and that $\|\cdot\|_{D}$ is a norm on the tensor space $\mathbf{V}_{D}={ }_{a} \bigotimes_{\alpha \in D} V_{\alpha}$ such that the tensor product map (3.1) is continuous. Let $\mathbf{v}, \mathbf{v}^{\prime} \in \mathfrak{M}_{\mathfrak{r}}\left(\mathbf{V}_{D}\right)$ be such that $\mathcal{U}(\mathbf{v}) \cap \mathcal{U}\left(\mathbf{v}^{\prime}\right) \neq \emptyset$. Then the bijective map

$$
\xi_{\mathbf{v}^{\prime}} \circ \xi_{\mathbf{v}}^{-1}: \xi_{\mathbf{v}}\left(\mathcal{U}(\mathbf{v}) \cap \mathcal{U}\left(\mathbf{v}^{\prime}\right)\right) \rightarrow \xi_{\mathbf{v}^{\prime}}\left(\mathcal{U}(\mathbf{v}) \cap \mathcal{U}\left(\mathbf{v}^{\prime}\right)\right)
$$

is $\mathcal{C}^{\infty}$-Fréchet differentiable.

Proof. Let $\mathbf{w} \in \mathcal{U}(\mathbf{v}) \cap \mathcal{U}\left(\mathbf{v}^{\prime}\right)$ be such that $\xi_{\mathbf{v}}(\mathbf{w})=\left(\mathfrak{L}, \mathbf{u}\left(C^{(D)}\right)\right)$ and $\xi_{\mathbf{v}^{\prime}}(\mathbf{w})=\left(\mathfrak{L}^{\prime}, \mathbf{u}^{\prime}\left(E^{(D)}\right)\right.$, that is,

$$
\left(\xi_{\mathbf{v}^{\prime}} \circ \xi_{\mathbf{v}}^{-1}\right)\left(\mathfrak{L}, \mathbf{u}\left(C^{(D)}\right)\right)=\left(\mathfrak{L}^{\prime}, \mathbf{u}^{\prime}\left(E^{(D)}\right)\right) .
$$

Since $\mathbf{w} \in \mathcal{U}(\mathbf{v}) \cap \mathcal{U}\left(\mathbf{v}^{\prime}\right)$ then

$$
\varrho_{\mathfrak{r}}(\mathbf{w})=\left(U_{\alpha}^{\min }(\mathbf{w})\right)_{\alpha \in D} \in\left(\underset{\alpha \in D}{X} \mathbb{G}\left(W_{\alpha}^{\min }(\mathbf{v}), V_{\alpha}\right)\right) \cap\left(\underset{\alpha \in D}{X} \mathbb{G}\left(W_{\alpha}^{\min }\left(\mathbf{v}^{\prime}\right), V_{\alpha}\right)\right)
$$

and

$$
\left.\left(\Psi_{\mathbf{v}^{\prime}} \circ \Psi_{\mathbf{v}}^{-1}\right)\left(\Psi_{\mathbf{v}}\left(\left(U_{\alpha}^{\min }(\mathbf{w})\right)_{\alpha \in D}\right)\right)=\Psi_{\mathbf{v}^{\prime}}\left(U_{\alpha}^{\min }(\mathbf{w})\right)_{\alpha \in D}\right),
$$

that is,

$$
\left(\Psi_{\mathbf{v}^{\prime}} \circ \Psi_{\mathbf{v}}^{-1}\right)(\mathfrak{L})=\mathfrak{L}^{\prime}
$$

Hence

$$
\xi_{\mathbf{v}^{\prime}}(\mathbf{w})=\left(\left(\Psi_{\mathbf{v}^{\prime}} \circ \Psi_{\mathbf{v}}^{-1}\right)(\mathfrak{L}), \mathbf{u}^{\prime}\left(E^{(D)}\right)\right),
$$

where $\Psi_{\mathbf{v}^{\prime}} \circ \Psi_{\mathbf{v}}^{-1}$ is an analytic map. On the other hand, since

$$
\mathbf{w}=\xi_{\mathbf{v}}^{-1}\left(\mathfrak{L}, C^{(D)}\right)=\left(\bigotimes_{\alpha \in D} \exp \left(L_{\alpha}\right)\right)\left(\mathbf{u}\left(C^{(D)}\right)\right)=\xi_{\mathbf{v}^{\prime}}^{-1}\left(\mathfrak{L}^{\prime}, E^{(D)}\right)=\left(\bigotimes_{\alpha \in D} \exp \left(L_{\alpha}^{\prime}\right)\right)\left(\mathbf{u}^{\prime}\left(E^{(D)}\right)\right),
$$

we have

$$
\mathbf{u}^{\prime}\left(E^{(D)}\right)=\left(\bigotimes_{\alpha \in D} \exp \left(-L_{\alpha}^{\prime}\right) \circ \exp \left(L_{\alpha}\right)\right)\left(\mathbf{u}\left(C^{(D)}\right)\right)=\left(\bigotimes_{\alpha \in D} \exp \left(L_{\alpha}-L_{\alpha}^{\prime}\right)\right)\left(\mathbf{u}\left(C^{(D)}\right)\right),
$$

because from Proposition 2.11 $L_{\alpha} \circ L_{\alpha}^{\prime}=L_{\alpha}^{\prime} \circ L_{\alpha}=0$ holds. In consequence,

$$
\mathbf{u}^{\prime}\left(E^{(D)}\right)=f\left(\mathfrak{L}, \mathbf{u}\left(C^{(D)}\right)\right):=\left(\bigotimes_{\alpha \in D} \exp \left(L_{\alpha}-\left(\Psi_{\mathbf{v}^{\prime}}^{(\alpha)} \circ\left(\Psi_{\mathbf{v}}^{(\alpha)}\right)^{-1}\right)\left(L_{\alpha}\right)\right)\left(\mathbf{u}\left(C^{(D)}\right)\right)\right.
$$

where

$$
f: \underset{\alpha \in D}{X} \mathcal{L}\left(U_{\alpha}^{\min }(\mathbf{v}), W_{\alpha}^{\min }(\mathbf{v})\right) \times \mathfrak{M}_{\mathfrak{r}}\left({ }_{a} \bigotimes_{\alpha \in D} U_{\alpha}^{\min }(\mathbf{v})\right) \rightarrow \mathfrak{M}_{\mathfrak{r}}\left({ }_{a} \bigotimes_{\alpha \in D} U_{\alpha}^{\min }\left(\mathbf{v}^{\prime}\right)\right) .
$$

To prove the lemma we claim that the map $f$ is $\mathcal{C}^{\infty}$-Fréchet differentiable.

Recall that for each $\alpha \in D$ the map given by

$$
L_{\alpha}^{\prime}=\left(\Psi_{\mathbf{v}^{\prime}}^{(\alpha)} \circ\left(\Psi_{\mathbf{v}}^{(\alpha)}\right)^{-1}\right)\left(L_{\alpha}\right)
$$


is analytic because $\mathbb{G}_{r_{\alpha}}\left(V_{\alpha}\right)$ is an analytic Banach manifold. Since we can identify the linear space $\mathcal{L}\left(U_{\alpha}^{\min }(\mathbf{v}), W_{\alpha}^{\min }(\mathbf{v})\right)$ with a sub-algebra of $\mathcal{L}\left(V_{\alpha_{\|\cdot\|_{\alpha}}}, V_{\alpha_{\|\cdot\|_{\alpha}}}\right)$, from Example 2.20, we know that

$$
\exp : \mathcal{L}\left(U_{\alpha}^{\min }(\mathbf{v}), W_{\alpha}^{\min }(\mathbf{v})\right) \rightarrow \operatorname{GL}\left(\mathcal{L}\left(U_{\alpha}^{\min }(\mathbf{v}), W_{\alpha}^{\min }(\mathbf{v})\right)\right)
$$

is analytic for each $\alpha \in D$. In consequence, the map

$$
L_{\alpha} \mapsto \exp \left(L_{\alpha}-\left(\Psi_{\mathbf{v}^{\prime}}^{(\alpha)} \circ\left(\Psi_{\mathbf{v}}^{(\alpha)}\right)^{-1}\right)\left(L_{\alpha}\right)\right)
$$

is also analytic for each $\alpha \in D$. Finally, we conclude by using Proposition 3.5 that the map

$$
\underset{\alpha \in D}{\times} \mathcal{L}\left(U_{\alpha}^{\min }(\mathbf{v}), W_{\alpha}^{\min }(\mathbf{v})\right) \rightarrow \mathcal{L}\left({ }_{a} \bigotimes_{\alpha \in D} U_{\alpha}^{\min }(\mathbf{v}), \mathbf{V}_{D}\right)
$$

given by

$$
\left.\left(L_{\alpha}\right)_{\alpha \in D} \mapsto \bigotimes_{\alpha \in D} \exp \left(L_{\alpha}-\left(\Psi_{\mathbf{v}^{\prime}}^{(\alpha)} \circ\left(\Psi_{\mathbf{v}}^{(\alpha)}\right)^{-1}\right)\left(L_{\alpha}\right)\right)\right|_{U_{\alpha}^{\min }(\mathbf{v})}
$$

is $\mathcal{C}^{\infty}$-Fréchet differentiable. Observe that $f$ can be written by using the evaluation map

$$
\text { eval : } \mathcal{L}\left({ }_{a} \bigotimes_{\alpha \in D} U_{\alpha}^{\min }(\mathbf{v}),{ }_{a} \bigotimes_{\alpha \in D} U_{\alpha}^{\min }\left(\mathbf{v}^{\prime}\right)\right) \times{ }_{a} \bigotimes_{k \in D} U_{k}^{\min }(\mathbf{v}) \rightarrow{ }_{a} \bigotimes_{k \in D} U_{k}^{\min }\left(\mathbf{v}^{\prime}\right)
$$

given by

$$
\operatorname{eval}\left(F, \sum_{\substack{1 \leq i_{\alpha} \leq r_{\alpha} \\ \alpha \in D}} E_{\left(i_{\alpha}\right)_{\alpha \in D}}^{(D)} \bigotimes_{\alpha \in D} u_{i_{\alpha}}^{(\alpha)}\right)=F\left(\sum_{\substack{1 \leq i_{\alpha} \leq r_{\alpha} \\ \alpha \in D}} E_{\left(i_{\alpha}\right)_{\alpha} \in D}^{(D)} u_{i_{\alpha}}^{(\alpha)}\right),
$$

which is multilinear and continuous. From Proposition 2.1, it is also $\mathcal{C}^{\infty}$-Fréchet differentiable. Since

$$
f\left(\mathfrak{L}, \mathbf{u}\left(C^{(D)}\right)\right)=\operatorname{eval}\left(\left(\bigotimes_{\alpha \in D} \exp \left(L_{\alpha}-\left(\Psi_{\mathbf{v}^{\prime}}^{(\alpha)} \circ\left(\Psi_{\mathbf{v}}^{(\alpha)}\right)^{-1}\right)\left(L_{\alpha}\right)\right)\right), \mathbf{u}\left(C^{(D)}\right)\right),
$$

the claim follows. We recall that

$$
\mathfrak{M}_{\mathfrak{r}}\left({ }_{a} \bigotimes_{k \in D} U_{k}^{\min }\left(\mathbf{v}^{\prime}\right)\right)=\mathfrak{M}_{\mathfrak{r}}\left({ }_{a} \bigotimes_{k \in D} U_{k}^{\min }(\mathbf{v})\right)=\mathbb{R}_{*}^{\times_{\alpha \in D} r_{\alpha}}
$$

Thus the lemma is proved.

Remark 3.15 Observe that if we assume that $\left(V_{\alpha},\|\cdot\|_{\alpha}\right)$ is a complex Banach space for each $\alpha \in D$ and $\|\cdot\|_{D}$ is a norm on the complex tensor space $\mathbf{V}_{D}={ }_{a} \bigotimes_{\alpha \in D} V_{\alpha}$ such that the tensor product map (3.1) is continuous, from Proposition 3.4, we have that the extension of the tensor product map (3.1) is analytic. Moreover, the map

$$
\underset{\alpha \in D}{X} \mathcal{L}\left(U_{\alpha}^{\min }(\mathbf{v}), W_{\alpha}^{\min }(\mathbf{v})\right) \rightarrow \underset{\alpha \in D}{X} \mathcal{L}\left(U_{\alpha}^{\min }(\mathbf{v}), V_{\alpha}\right), \quad \mathfrak{L}=\left(L_{\alpha}\right)_{\alpha \in D} \mapsto\left(i d_{\alpha}+L_{\alpha}\right)_{\alpha \in D}=\left(\exp \left(L_{\alpha}\right)\right)_{\alpha \in D}
$$

between the product of complex Banach spaces is clearly analytic. In consequence, under the above assumptions it can be shown that the bijective map $\xi_{\mathbf{v}^{\prime}} \circ \xi_{\mathbf{v}}^{-1}$ is analytic.

Before stating the next result we recall the definition of a fibre bundle.

Definition $3.16 A \mathcal{C}^{k}$-fibre bundle $(E, B, \pi)$, where $k \geq 0$, with typical fibre $F$ (a given manifold) is a $\mathcal{C}^{k}$ surjective morphism of $\mathcal{C}^{k}$ manifolds $\pi: E \rightarrow B$ which is locally a product, that is, the $\mathcal{C}^{k}$-manifold $B$ has an open atlas $\left\{\left(U_{\alpha}, \xi_{\alpha}\right)\right\}_{\alpha \in A}$ such that for each $\alpha \in A$ there is a $\mathcal{C}^{k}$ diffeomorphism $\chi_{\alpha}: \pi^{-1}\left(U_{\alpha}\right) \rightarrow U_{\alpha} \times F$ such that $p_{\alpha} \circ \chi_{\alpha}=\pi$, where $p_{\alpha}: U_{\alpha} \times F \rightarrow U_{\alpha}$ is the projection. The $\mathcal{C}^{k}$ manifolds $E$ and $B$ are called the total space and base of the fibre bundle, respectively. For each $b \in B, \pi^{-1}(b)=E_{b}$ is called the fibre over $b$. The $\mathcal{C}^{k}$ diffeomorphisms $\chi_{\alpha}$ are called fibre bundle charts. 
Theorem 3.17 Assume that $\left(V_{\alpha},\|\cdot\|_{\alpha}\right)$ is a normed space for each $\alpha \in D$ and that $\|\cdot\|_{D}$ is a norm on the tensor space $\mathbf{V}_{D}={ }_{a} \bigotimes_{\alpha \in D} V_{\alpha}$ such that the tensor product map (3.1) is continuous. Then the collection $\left\{\mathcal{U}(\mathbf{v}), \xi_{\mathbf{v}}\right\}_{\mathbf{v} \in \mathfrak{M}_{\mathfrak{r}}\left(\mathbf{V}_{D}\right)}$ is a $\mathcal{C}^{\infty}$-atlas for $\mathfrak{M}_{\mathfrak{r}}\left(\mathbf{V}_{D}\right)$ and hence it is a $\mathcal{C}^{\infty}$-Banach manifold modelled on a Banach space

$$
\left(\underset{\alpha \in D}{X} \mathcal{L}\left(U_{\alpha}, W_{\alpha}\right)\right) \times \mathbb{R}^{\times_{\alpha \in D} r_{\alpha}},
$$

here $U_{\alpha} \in \mathbb{G}_{r_{\alpha}}\left(V_{\alpha}\right)$ and $V_{\alpha_{\|\cdot\|_{\alpha}}}=U_{\alpha} \oplus W_{\alpha}$, where $V_{\alpha_{\|\cdot\|_{\alpha}}}$ is the completion of $V_{\alpha}$ for $\alpha \in D$. Moreover,

$$
\left(\mathfrak{M}_{\mathfrak{r}}\left(\mathbf{V}_{D}\right), \underset{\alpha \in D}{\chi} \mathbb{G}_{r_{\alpha}}\left(V_{\alpha}\right), \varrho_{\mathfrak{r}}\right)
$$

is a $\mathcal{C}^{\infty}$-fibre bundle with typical fibre $\mathbb{R}_{*}^{\times_{\alpha \in D} r_{\alpha}}$.

Proof. Since $\left\{\left(\mathcal{U}(\mathbf{v}), \xi_{\mathbf{v}}\right)\right\}_{\mathbf{v} \in \mathfrak{M}_{\mathfrak{r}}\left(\mathbf{V}_{D}\right)}$ satisfies AT1, Lemma 3.12 implies AT2 and AT3 follows from Lemma 3.14 we obtain the first statement. To prove the second one we observe that the local chart system $\left\{\left(\mathcal{U}(\mathbf{v}), \xi_{\mathbf{v}}\right)\right\}$ for the manifold $\mathfrak{M}_{\mathfrak{r}}\left(\mathbf{V}_{D}\right)$ allows us to write the morphism

$$
\varrho_{\mathfrak{r}}: \mathfrak{M}_{\mathfrak{r}}\left(\mathbf{V}_{D}\right) \rightarrow \underset{\alpha \in D}{\chi} \mathbb{G}_{r_{\alpha}}\left(V_{\alpha}\right), \quad \mathbf{v} \mapsto\left(U_{\alpha}^{\min }(\mathbf{v})\right)_{\alpha \in D}
$$

locally as a map

$$
\left(\underset{\alpha \in D}{X} \mathcal{L}\left(U_{\alpha}^{\min }(\mathbf{v}), W_{\alpha}^{\min }(\mathbf{v})\right)\right) \times \mathbb{R}_{*}^{\times_{\alpha \in D} r_{\alpha}} \rightarrow \underset{\alpha \in D}{X} \mathcal{L}\left(U_{\alpha}^{\min }(\mathbf{v}), W_{\alpha}^{\min }(\mathbf{v})\right),
$$

given by

$$
\left(\left(L_{\alpha}\right)_{\alpha \in D}, E^{(D)}\right) \mapsto\left(L_{\alpha}\right)_{\alpha \in D}
$$

Thus, $\varrho_{\mathfrak{r}}$ is a $C^{\infty}$-surjective morphism. Moreover, by construction of the atlases, for each $\mathbf{v} \in \mathfrak{M}_{\mathfrak{r}}\left(\mathbf{V}_{D}\right)$ the $\operatorname{map} \chi_{\mathbf{v}}:=\left(\Psi_{\mathbf{v}} \times i d_{\mathbb{R}_{*}^{\times} \in D^{r_{\alpha}}}\right) \circ \xi_{\mathbf{v}}$ where

$$
\chi_{\mathbf{v}}: \mathcal{U}(\mathbf{v})=\varrho_{\mathfrak{r}}^{-1}\left(\underset{\alpha \in D}{X} \mathbb{G}\left(W_{\alpha}^{\min }(\mathbf{v}), V_{\alpha}\right)\right) \rightarrow\left(\underset{\alpha \in D}{X} \mathbb{G}\left(W_{\alpha}^{\min }(\mathbf{v}), V_{\alpha}\right)\right) \times \mathbb{R}_{*}^{\times_{\alpha \in D} r_{\alpha}}
$$

is a $C^{\infty}$-diffeomorphism satisfying $\pi_{\mathbf{v}} \circ \chi_{\mathbf{v}}=\varrho_{\mathfrak{r}}$ where

$$
\pi_{\mathbf{v}}:\left(\underset{\alpha \in D}{X} \mathbb{G}\left(W_{\alpha}^{\min }(\mathbf{v}), V_{\alpha}\right)\right) \times \mathbb{R}_{*}^{\times_{\alpha \in D} r_{\alpha}} \rightarrow \underset{\alpha \in D}{X} \mathbb{G}\left(W_{\alpha}^{\min }(\mathbf{v}), V_{\alpha}\right), \quad\left(\left(U_{\alpha}\right)_{\alpha \in D}, E^{(D)}\right) \mapsto\left(U_{\alpha}\right)_{\alpha \in D}
$$

In consequence, the second statement is proved.

Remark 3.18 We point out that for $d=2$ the typical fibre is the Lie group GL( $\left.\mathbb{R}^{r}\right)$ for some $r \geq 1$ and for $\mathfrak{r}=\mathbf{1}$ (and any $d \geq 2$ ) the typical fibre is the Lie group $\mathrm{GL}(\mathbb{R})=\mathbb{R} \backslash\{0\}$. Then in both cases we have that the fibre bundle is a principal bundle, that is, a fibre bundle which has as a typical fibre a Lie group.

Remark 3.19 Assume that $\left(V_{\alpha},\|\cdot\|_{\alpha}\right)$ is a complex Banach space for each $\alpha \in D$ and $\|\cdot\|_{D}$ is a norm on the complex tensor space $\mathbf{V}_{D}={ }_{a} \bigotimes_{\alpha \in D} V_{\alpha}$ such that the tensor product map (3.1) is continuous. From Remark 3.15 we have that the collection $\left\{\mathcal{U}(\mathbf{v}), \xi_{\mathbf{v}}\right\}_{\mathbf{v} \in \mathfrak{M}_{\mathfrak{r}}\left(\mathbf{V}_{D}\right)}$ is an analytic atlas for $\mathfrak{M}_{\mathfrak{r}}\left(\mathbf{V}_{D}\right)$ and hence it is an analytic Banach manifold modelled on a Banach space

$$
\left(\underset{\alpha \in D}{X} \mathcal{L}\left(U_{\alpha}, W_{\alpha}\right)\right) \times \mathbb{C}^{\times_{\alpha \in D} r_{\alpha}}
$$

here $U_{\alpha} \in \mathbb{G}_{r_{\alpha}}\left(V_{\alpha}\right)$ and $V_{\alpha}=U_{\alpha} \oplus W_{\alpha}$ for $\alpha \in D$. 
We point out that the norm $\|\cdot\|_{D}$ in $\mathbf{V}_{D}$ is only used in the proof of Lemma 3.14 in order to endow the finite-dimensional tensor space ${ }_{a} \bigotimes_{\alpha \in D} U_{\alpha}^{\min }(\mathbf{v})$ with a structure of finite-dimensional Banach space for each $\mathbf{v} \in \mathfrak{M}_{\mathfrak{r}}\left(\mathbf{V}_{D}\right)$. Thus, the geometric structure of manifold is independent of the choice of the norm $\|\cdot\|_{D}$ over the tensor space $\mathbf{V}_{D}$. We illustrate this assertion with the following example.

Example 3.20 Let $V_{1_{\|\cdot\|_{1}}}:=H^{1, p}\left(I_{1}\right)$ and $V_{2_{\|\cdot\|_{2}}}=H^{1, p}\left(I_{2}\right)$, with $\|\cdot\|_{\alpha}=\|\cdot\|_{1, p, I_{\alpha}}$, and $1 \leq p<\infty$. Take $\mathbf{V}_{D}:=H^{1, p}\left(I_{1}\right) \otimes_{a} H^{1, p}\left(I_{2}\right)$. Now, we can consider as ambient Banach space either

$$
\overline{\mathbf{V}}_{D}^{\|\cdot\|_{D, 1}}:=H^{1, p}\left(I_{1} \times I_{2}\right)
$$

with $\|\cdot\|_{D, 1}=\|\cdot\|_{1, p}$, or

$$
\overline{\mathbf{V}}_{D}^{\|\cdot\|_{D, 2}}=H^{1, p}\left(I_{1}\right) \otimes_{\|\cdot\|_{D, 2}} H^{1, p}\left(I_{2}\right),
$$

where $\|\cdot\|_{D, 2}:=\|\cdot\|_{(0,1), p}$ is the norm given by

$$
\|f\|_{(0,1), p}:=\left(\|f\|_{p}^{p}+\left\|\frac{\partial f}{\partial x_{2}}\right\|_{p}^{p}\right)^{1 / p} .
$$

The tensor product map (3.1) is continuous for both norms (see Examples 4.41 and 4.42 in [19]) and hence from Theorem 3.17 we obtain that for each $r \geq 1$ the set $\mathfrak{M}_{(r, r)}\left(\mathbf{V}_{D}\right)$ is a $\mathcal{C}^{\infty}$-Banach manifold modelled on

$$
\mathcal{L}\left(U_{1}, W_{1}\right) \times \mathcal{L}\left(U_{2}, W_{2}\right) \times \mathrm{GL}\left(\mathbb{R}^{r}\right),
$$

here $U_{i} \in \mathbb{G}_{r}\left(H^{1, p}\left(I_{i}\right)\right)$ and $H^{1, p}\left(I_{i}\right)=U_{i} \oplus W_{i}$ for $i=1,2$.

The next result gives us the conditions to have a Hilbert manifold.

Corollary 3.21 Assume that $\left(V_{\alpha},\|\cdot\|_{\alpha}\right)$ is a normed space such that $V_{\alpha_{\|\cdot\|_{\alpha}}}$ is a Hilbert space for each $\alpha \in D$ and let $\|\cdot\|_{D}$ be a norm on the tensor space $\mathbf{V}_{D}={ }_{a} \bigotimes_{\alpha \in D} V_{\alpha}$ such that the tensor product map (3.1) is continuous. Then $\mathfrak{M}_{\mathfrak{r}}\left(\mathbf{V}_{D}\right)$ is a $\mathcal{C}^{\infty}$-Hilbert manifold modelled on a Hilbert space

$$
\underset{\alpha \in D}{X} W_{\alpha}^{r_{\alpha}} \times \mathbb{R}^{\times_{\alpha \in D} r_{\alpha}},
$$

here $V_{\alpha_{\|\cdot\|_{\alpha}}}=U_{\alpha} \oplus W_{\alpha}$, for some $U_{\alpha} \in \mathbb{G}_{r_{\alpha}}\left(V_{\alpha}\right)$ for $\alpha \in D$.

Proof. We can identify each $L_{\alpha} \in \mathcal{L}\left(U_{\alpha}^{\min }(\mathbf{v}), W_{\alpha}^{\min }(\mathbf{v})\right)$ with a set of vectors $\left(w_{s_{\alpha}}^{(\alpha)}\right)_{s_{\alpha}=1}^{s_{\alpha}=r_{\alpha}} \in W_{\alpha}^{\min }(\mathbf{v})^{r_{\alpha}}$, where $w_{s_{\alpha}}^{(\alpha)}=L_{\alpha}\left(u_{s_{\alpha}}^{(\alpha)}\right)$ and $U_{\alpha}^{\min }(\mathbf{v})=\operatorname{span}\left\{u_{1}^{(\alpha)}, \ldots, u_{r_{\alpha}}^{(\alpha)}\right\}$ for $\alpha \in D$. Thus we can identify each $\left(\mathfrak{L}, C^{(D)}\right) \in$ $\xi_{\mathbf{v}}(\mathcal{U}(\mathbf{v}))$ with a pair

$$
\left(\mathcal{W}, C^{(D)}\right) \in \underset{\alpha \in D}{X} W_{\alpha}^{\min }(\mathbf{v})^{r_{\alpha}} \times \mathbb{R}_{*}^{\times_{\alpha \in D} r_{\alpha}}
$$

where $\mathcal{W}:=\left(\left(w_{i_{\alpha}}^{(\alpha)}\right)_{i_{\alpha}=1}^{r_{\alpha}}\right)_{\alpha \in D}$. Take $\times_{\alpha \in D} W_{\alpha}^{\min }(\mathbf{v})^{r_{\alpha}} \times \mathbb{R}_{*}^{\times_{\alpha \in D} r_{\alpha}}$ an open subset of the Hilbert space $\times_{\alpha \in D} W_{\alpha}^{\min }(\mathbf{v})^{r_{\alpha}} \times \mathbb{R}^{\times_{\alpha \in D} r_{\alpha}}$ endowed with the inner product norm

$$
\left\|\left(\mathcal{W}, C^{(D)}\right)\right\|_{\times, \mathbf{v}}^{2}:=\left\|C^{(D)}\right\|_{F}^{2}+\sum_{\alpha \in D} \sum_{i_{\alpha}=1}^{r_{\alpha}}\left\|w_{i_{\alpha}}^{(\alpha)}\right\|_{\alpha}^{2},
$$

with $\|\cdot\|_{F}$ the Frobenius norm. It allows us to define local charts, also denoted by $\xi_{\mathbf{v}}$, by

$$
\xi_{\mathbf{v}}^{-1}: \underset{\alpha \in D}{X} W_{\alpha}^{\min }(\mathbf{v})^{r_{\alpha}} \times \mathbb{R}_{*}^{\times_{\alpha \in D} r_{\alpha}} \longrightarrow \mathcal{U}(\mathbf{v}),
$$

where $\xi_{\mathbf{v}}^{-1}\left(\mathcal{W}, C^{(D)}\right)=\mathbf{w}$, putting $L_{\alpha}\left(u_{i_{\alpha}}^{(\alpha)}\right)=w_{i_{\alpha}}^{(\alpha)}, 1 \leq i_{\alpha} \leq r_{\alpha}$ and $\alpha \in D$. Since each local chart is defined over an open subset of the Hilbert space $\times_{\alpha \in D} W_{\alpha}^{\min }(\mathbf{v})^{r_{\alpha}} \times \mathbb{R}^{\times_{\alpha \in D} r_{\alpha}}$, the corollary follows. 
Using the definition of the local charts for the manifold $\mathfrak{M}_{\mathfrak{r}}\left(\mathbf{V}_{D}\right)$, we can identify its tangent space at $\mathbf{v}$ with $\mathbb{T}_{\mathbf{v}}\left(\mathfrak{M}_{\mathfrak{r}}\left(\mathbf{V}_{D}\right)\right):=\times_{\alpha \in D} \mathcal{L}\left(U_{\alpha}^{\min }(\mathbf{v}), W_{\alpha}^{\min }(\mathbf{v})\right) \times \mathbb{R}^{\times_{\alpha \in D} r_{\alpha}}$. We will consider $\mathbb{T}_{\mathbf{v}}\left(\mathfrak{M}_{\mathfrak{r}}\left(\mathbf{V}_{D}\right)\right)$ endowed with the product norm

$$
\|\left(\left|\left(\mathfrak{L}, C^{(D)}\right)\right|\left\|_{\mathbf{v}}:=\right\| C^{(D)}\left\|_{F}+\sum_{\alpha \in D}\right\| L_{\alpha} \|_{W_{\alpha}^{\min }(\mathbf{v}) \leftarrow U_{\alpha}^{\min }(\mathbf{v})} .\right.
$$

Finally, the fact that $\mathbf{V}_{D}=\bigcup_{\mathfrak{r} \in \mathcal{A D}\left(\mathbf{V}_{D}\right)} \mathfrak{M}_{\mathfrak{r}}\left(\mathbf{V}_{D}\right)$ allows us to state the following.

Corollary 3.22 Assume that $\left(V_{\alpha},\|\cdot\|_{\alpha}\right)$ is a normed space for each $\alpha \in D$ and that $\|\cdot\|_{D}$ is a norm on the tensor space $\mathbf{V}_{D}={ }_{a} \bigotimes_{\alpha \in D} V_{\alpha}$ such that the tensor product map (3.1) is continuous. Then the algebraic tensor space $\mathbf{V}_{D}$ is a $\mathcal{C}^{\infty}$-Banach manifold not modelled on a particular Banach space.

\section{The manifold of tensors in Tucker format with fixed rank and its natural ambient tensor Banach space}

Consider the tensor space $\mathbf{V}_{D}={ }_{a} \bigotimes_{\alpha \in D} V_{\alpha}$ and assume that for each $\alpha \in D$ the vector space $V_{\alpha}$ is a normed space with a norm $\|\cdot\|_{\alpha}$. We start with a brief discussion about the choice of the ambient manifold for $\mathfrak{M}_{\mathfrak{r}}\left(\mathbf{V}_{D}\right)$. Recall that in Example 3.20 we have two norms $\|\cdot\|_{D, 1}$ and $\|\cdot\|_{D, 2}$ on $\mathbf{V}_{D}$ such that the tensor product map (3.1) is continuous for both norms. Then we have two natural embeddings $\mathbf{V}_{D} \subset \overline{\mathbf{V}_{D}}\|\cdot\|_{D, 1}$ and $\mathbf{V}_{D} \subset \overline{\mathbf{V}_{D}}\|\cdot\|_{D, 2}$. In this context a natural question about the choice of a norm $\|\cdot\|_{D}$ for the algebraic tensor space $\mathbf{V}_{D}$ appears: What is the good choice for this norm to show that $\mathfrak{M}_{\mathfrak{r}}\left(\mathbf{V}_{D}\right)$ is an immersed submanifold?

More precisely, assume that $\left(V_{\alpha},\|\cdot\|_{\alpha}\right)$ is a normed space for each $\alpha \in D$ and let $\|\cdot\|_{D}$ be a norm on the tensor space $\mathbf{V}_{D}={ }_{a} \bigotimes_{\alpha \in D} V_{\alpha}$ such that the tensor product map (3.1) is continuous. Then we have a natural ambient space for $\mathfrak{M}_{\mathfrak{r}}\left(\mathbf{V}_{D}\right)$ given by a Banach tensor space $\overline{\mathbf{V}_{D}}\|\cdot\|_{D}=\mathbf{V}_{D_{\|\cdot\|_{D}}}$. Since the natural inclusion

$$
\mathfrak{i}: \mathfrak{M}_{\mathfrak{r}}\left(\mathbf{V}_{D}\right) \longrightarrow \mathbf{V}_{D_{\|\cdot\|_{D}}}
$$

given by $\mathfrak{i}(\mathbf{v})=\mathbf{v}$, is an injective map we will study $\mathfrak{i}$ as a function between Banach manifolds. To this end we recall the definition of an immersion between manifolds.

Definition 4.1 Let $F: X \rightarrow Y$ be a morphism between Banach manifolds and let $x \in X$. We shall say that $F$ is an immersion at $x$ if there exists an open neighbourhood $X_{x}$ of $x$ in $X$ such that the restriction of $F$ to $X_{x}$ induces an isomorphism from $X_{x}$ onto a submanifold of $Y$. We say that $F$ is an immersion if it is an immersion at each point of $X$.

Our next step is to recall the definition of the differential as a morphism which gives a linear map between the tangent spaces of the manifolds involved with the morphism.

Definition 4.2 Let $X$ and $Y$ be two Banach manifolds. Let $F: X \rightarrow Y$ be a $\mathcal{C}^{r}$ morphism, i.e.,

$$
\psi \circ F \circ \varphi^{-1}: \varphi(U) \rightarrow \psi(W)
$$

is a $\mathcal{C}^{r}$-Fréchet differentiable map, where $(U, \varphi)$ is a chart in $X$ at $x$ and $(W, \psi)$ is a chart in $Y$ at $F(x)$. For $x \in X$, we define

$$
\mathrm{T}_{x} F: \mathbb{T}_{x}(X) \longrightarrow \mathbb{T}_{F(x)}(Y), \quad v \mapsto\left[\left(\psi \circ F \circ \varphi^{-1}\right)^{\prime}(\varphi(x))\right] v .
$$

For Banach manifolds we have the following criterion for immersions (see Theorem 3.5.7 in [28]).

Proposition 4.3 Let $X, Y$ be Banach manifolds of class $\mathcal{C}^{p}(p \geq 1)$. Let $F: X \rightarrow Y$ be a $\mathcal{C}^{p}$ morphism and $x \in X$. Then $F$ is an immersion at $x$ if and only if $\mathrm{T}_{x} F$ is injective and $\mathrm{T}_{x} F\left(\mathbb{T}_{x}(X)\right) \in \mathbb{G}_{(}\left(\mathbb{T}_{F(x)}(Y)\right)$. 
A concept related to an immersion between Banach manifolds is introduced in the following definition.

Definition 4.4 Assume that $X$ and $Y$ are Banach manifolds and let $f: X \longrightarrow Y$ be a $\mathcal{C}^{r}$ morphism. If $f$ is an injective immersion, then $f(X)$ is called an immersed submanifold of $Y$.

In consequence, to prove that the standard inclusion map $\mathfrak{i}$ is an immersion we shall prove, under the appropriate conditions, that if $\mathfrak{i}$ is a differentiable morphism then for each $\mathbf{v} \in \mathfrak{M}_{\mathfrak{r}}\left(\mathbf{V}_{D}\right)$ the linear map $T_{\mathbf{v}} \mathfrak{i}$ is injective and $T_{\mathbf{v}} \mathfrak{i}\left(\mathbb{T}_{\mathbf{v}}\left(\mathfrak{M}_{\mathfrak{r}}\left(\mathbf{V}_{D}\right)\right)\right)$ belongs to $\mathbb{G}\left(\mathbf{V}_{D_{\|\cdot\|_{D}}}\right)$.

\subsection{The linear map $T_{v} i$ is injective}

To describe $\mathfrak{i}$ as a morphism, we proceed as follows. Given $\mathbf{v}=\sum_{\substack{1 \leq i_{\alpha} \leq r_{\alpha} \\ \alpha \in D}} C_{\left(i_{\alpha}\right)_{\alpha \in D}}^{(D)} \bigotimes_{\alpha \in D} u_{i_{\alpha}}^{(\alpha)} \in \mathfrak{M}_{\mathfrak{r}}\left(\mathbf{V}_{D}\right)$, we consider $\mathcal{U}(\mathbf{v})$, a neighbourhood of $\mathbf{v}$, and

$$
\left(\mathfrak{i} \circ \xi_{\mathbf{v}}^{-1}\right): \underset{\alpha \in D}{X} \mathcal{L}\left(U_{\alpha}^{\min }(\mathbf{v}), W_{\alpha}^{\min }(\mathbf{v})\right) \times \mathbb{R}_{*}^{\mathfrak{r}} \rightarrow \mathbf{V}_{\|\cdot\|_{D}} .
$$

From the proof of Lemma 3.14 the map $\left(\mathfrak{i} \circ \xi_{\mathbf{v}}^{-1}\right)$ is given by

$$
\left(\mathfrak{i} \circ \xi_{\mathbf{v}}^{-1}\right)\left(\mathfrak{L}, E^{(D)}\right)=\operatorname{eval}\left(\bigotimes_{\alpha \in D}\left(i d_{\alpha}+L_{\alpha}\right), \mathbf{u}\left(E^{(D)}\right)\right)=\sum_{\substack{1 \leq i_{\alpha} \leq r_{\alpha} \\ \alpha \in D}} E_{\left(i_{\alpha}\right)_{\alpha \in D}}^{(D)} \bigotimes_{\alpha \in D}\left(i d_{\alpha}+L_{\alpha}\right)\left(u_{i_{\alpha}}^{(\alpha)}\right) .
$$

Remark 4.5 Observe that it allows us to define a left local action of the Lie group $\times_{\alpha \in D} \operatorname{GL}\left(\mathcal{L}\left(U_{\alpha}^{\min }(\mathbf{v}), W_{\alpha}^{\min }(\mathbf{v})\right)\right)$ onto the local manifold $\mathcal{U}(\mathbf{v})$ as follows:

$$
\underset{\alpha \in D}{X} \operatorname{GL}\left(\mathcal{L}\left(U_{\alpha}^{\min }(\mathbf{v}), W_{\alpha}^{\min }(\mathbf{v})\right)\right) \times \mathcal{U}(\mathbf{v}) \rightarrow \mathcal{U}(\mathbf{v}), \quad\left(\left(\exp \left(L_{\alpha}\right)\right)_{\alpha \in D}, \mathbf{w}\right) \mapsto \bigotimes_{\alpha \in D} \exp \left(L_{\alpha}\right)(\mathbf{w}) .
$$

Moreover, we can also define a right local action using the Lie group $\times_{\alpha \in D} \operatorname{GL}\left(U_{\alpha}^{\min }(\mathbf{v})\right)$ by

$\mathcal{U}(\mathbf{v}) \times\left(\underset{\alpha \in D}{X} \operatorname{GL}\left(U_{\alpha}^{\min }(\mathbf{v})\right)\right) \rightarrow \mathcal{U}(\mathbf{v}), \quad\left(\bigotimes_{\alpha \in D} \exp \left(L_{\alpha}\right)\left(\mathbf{u}\left(E^{(D)}\right)\right),\left(G_{\alpha}\right)_{\alpha \in D}\right) \mapsto \bigotimes_{\alpha \in D}\left(\exp \left(L_{\alpha}\right) \circ G_{\alpha}\right)\left(\mathbf{u}\left(E^{(D)}\right)\right)$.

The next lemma describes the tangent map $T_{\mathbf{v}} i$.

Proposition 4.6 Assume that $\left(V_{\alpha},\|\cdot\|_{\alpha}\right)$ is a normed space for each $\alpha \in D$ and let $\|\cdot\|_{D}$ be a norm on the tensor space $\mathbf{V}_{D}={ }_{a} \bigotimes_{\alpha \in D} V_{\alpha}$ such that the tensor product map (3.1) is continuous. For $\mathbf{v}=$ $\sum_{\substack{1 \leq i_{\alpha} \leq r_{\alpha} \\ \alpha \in D}} C_{\left(i_{\alpha}\right)_{\alpha \in D}}^{(D)} \bigotimes_{\alpha \in D} u_{i_{\alpha}}^{(\alpha)} \in \mathfrak{M}_{\mathfrak{r}}\left(\mathbf{V}_{D}\right)$ the following statements hold.

(a) The map $\left(\mathfrak{i} \circ \xi_{\mathbf{v}}^{-1}\right)$ from $\times_{\alpha \in D} \mathcal{L}\left(U_{\alpha}^{\min }(\mathbf{v}), W_{\alpha}^{\min }(\mathbf{v})\right) \times \mathbb{R}^{\times_{\alpha \in D} r_{\alpha}}$ to $\mathbf{V}_{D_{\|\cdot\|_{D}}}$ is Fréchet differentiable, and hence

$$
\mathrm{T}_{\mathbf{v}} \mathfrak{i} \in \mathcal{L}\left(\mathbb{T}_{\mathbf{v}}\left(\mathfrak{M}_{\mathfrak{r}}\left(\mathbf{V}_{D}\right)\right), \mathbf{V}_{D_{\|\cdot\|_{D}}}\right)
$$

(b) Assume $\left(\dot{\mathfrak{L}}, \dot{C}^{(D)}\right) \in \mathbb{T}_{\mathbf{v}}\left(\mathfrak{M}_{\mathbf{r}}\left(\mathbf{V}_{D}\right)\right)$, where $\dot{C}^{(D)} \in \mathbb{R}^{\times_{\alpha \in D} r_{\alpha}}$ and $\dot{\mathfrak{L}}=\left(\dot{L}_{\alpha}\right)_{\alpha \in D}$ is in $\times_{\alpha \in D} \mathcal{L}\left(U_{\alpha}^{\min }(\mathbf{v}), W_{\alpha}^{\min }(\mathbf{v})\right)$. Then $\dot{\mathbf{w}}=\mathrm{T}_{\mathbf{v}} \mathfrak{i}\left(\dot{\mathfrak{L}}, \dot{C}^{(D)}\right)$ if and only if

$$
\dot{\mathbf{w}}=\sum_{\substack{1 \leq i_{\alpha} \leq r_{\alpha} \\ \alpha \in D}} \dot{C}_{\left(i_{\alpha}\right)_{\alpha \in D}}^{(D)} \bigotimes_{\alpha \in D} u_{i_{\alpha}}^{(\alpha)}+\sum_{\substack{1 \leq i_{\alpha} \leq r_{\alpha} \\ \alpha \in D}}\left(\dot{u}_{i_{\alpha}}^{(\alpha)} \otimes \mathbf{U}_{i_{\alpha}}^{(\alpha)}\right)
$$

where

$$
\mathbf{U}_{i_{\alpha}}^{(\alpha)}=\sum_{\substack{1 \leq i_{\beta} \leq r_{\beta} \\ \beta \in D \backslash\{\alpha\}}} C_{i_{\alpha},\left(i_{\beta}\right)_{\beta \in D \backslash\{\alpha\}}}^{(D)} \bigotimes_{\beta \in D} u_{i_{\beta}}^{(\beta)}
$$


Proof. To prove statement (a), from the results of section 3.3 we know that $\left(\mathfrak{i} \circ \xi_{\mathbf{v}}^{-1}\right)$ is $\mathcal{C}^{\infty}$-Fréchet differentiable and that $\xi_{\mathbf{v}}(\mathbf{v})=\left(\mathrm{o}, C^{(D)}\right)$. Now, to prove $(\mathrm{b})$ observe that

$$
\mathrm{T}_{\mathbf{v}} \mathfrak{i}: \underset{\alpha \in D}{\chi} \mathcal{L}\left(U_{\alpha}^{\min }(\mathbf{v}), W_{\alpha}^{\min }(\mathbf{v})\right) \times \mathbb{R}^{\times_{\alpha \in D} r_{\alpha}} \longrightarrow \mathbf{V}_{\|\cdot\|_{D}}
$$

is given by the chain rule:

$$
\begin{aligned}
& \mathrm{T}_{\mathbf{v}} \mathfrak{i}\left(\dot{\mathfrak{L}}, \dot{C}^{(D)}\right)=\left[\left(\mathfrak{i} \circ \xi_{\mathbf{v}}^{-1}\right)^{\prime}\left(\left(\mathfrak{i} \circ \xi_{\mathbf{v}}^{-1}\right)(\mathbf{v})\right)\right]\left(\dot{\mathfrak{L}}, \dot{C}^{(D)}\right) \\
& =\left[\left(\mathfrak{i} \circ \xi_{\mathbf{v}}^{-1}\right)^{\prime}\left(\mathcal{o}, C^{(D)}\right)\right]\left(\dot{\mathfrak{L}}, \dot{C}^{(D)}\right) \\
& =\sum_{\substack{1 \leq i_{\alpha} \leq r_{\alpha} \\
\alpha \in D}} \dot{C}_{\left(i_{\alpha}\right)_{\alpha \in D}}^{(D)} \bigotimes_{\alpha \in D} u_{i_{\alpha}}^{(\alpha)}+\sum_{\substack{1 \leq i_{\alpha} \leq r_{\alpha} \\
\alpha \in D}} \sum_{\substack{1 \leq i_{\beta} \leq r_{\beta} \\
\beta \in D \backslash\{\alpha\}}} C_{i_{\alpha},\left(i_{\beta}\right)_{\beta \in D} \backslash\{\alpha\}}^{(D)}\left(\dot{L}_{\alpha}\left(u_{i_{\alpha}}^{\alpha}\right) \otimes \bigotimes_{\substack{\beta \in D \\
\beta \neq \alpha}} u_{i_{\beta}}^{(\beta)}\right) \\
& =\sum_{\substack{1 \leq i_{\alpha} \leq r_{\alpha} \\
\alpha \in D}} \dot{C}_{\left(i_{\alpha}\right)_{\alpha \in D}}^{(D)} \bigotimes_{\alpha \in D} u_{i_{\alpha}}^{(\alpha)}+\sum_{\substack{1 \leq i_{\alpha} \leq r_{\alpha} \\
\alpha \in D}}\left(\dot{L}_{\alpha}\left(u_{i_{\alpha}}^{\alpha}\right) \otimes \mathbf{U}_{i_{\alpha}}^{(\alpha)}\right) .
\end{aligned}
$$

This implies statement (b).

In the next proposition we prove that $T_{\mathbf{v}} \mathfrak{i}$ is injective when we consider $\mathbf{v}$ in the manifold $\mathfrak{M}_{\mathbf{r}}\left(\mathbf{V}_{D}\right)$. It allows us to characterise the tangent space of $\mathfrak{M}_{\mathbf{r}}\left(\mathbf{V}_{D}\right)$ inside the tensor space $\mathbf{V}_{D_{\|\cdot\|_{D}}}$. We recall that from Remark 3.11 we have

$$
U_{D \backslash\{\alpha\}}^{\min }(\mathbf{v})=\operatorname{span}\left\{\mathbf{U}_{i_{\alpha}}^{(\alpha)}: 1 \leq i_{\alpha} \leq r_{\alpha}\right\}
$$

for $\alpha \in D$. In order to simplify notations we introduce the following definition. For each $\mathbf{v} \in \mathfrak{M}_{\mathfrak{r}}\left(\mathbf{V}_{D}\right)$ we denote by $\mathbf{Z}^{(D)}(\mathbf{v})$ the linear subspace in $\mathbf{V}_{D_{\|\cdot\|_{D}}}$ defined by

$$
\mathbf{Z}^{(D)}(\mathbf{v}):={ }_{a} \bigotimes_{\alpha \in D} U_{\alpha}^{\min }(\mathbf{v}) \oplus\left(\bigoplus_{\alpha \in D} W_{\alpha}^{\min }(\mathbf{v}) \otimes_{a} U_{D \backslash\{\alpha\}}^{\min }(\mathbf{v})\right)
$$

Proposition 4.7 Assume that $\left(V_{\alpha},\|\cdot\|_{\alpha}\right)$ is a normed space for each $\alpha \in D$ and let $\|\cdot\|_{D}$ be a norm on the tensor space $\mathbf{V}_{D}={ }_{a} \bigotimes_{\alpha \in D} V_{\alpha}$ such that the tensor product map (3.1) is continuous. Let $\mathbf{v} \in \mathfrak{M}_{\mathfrak{r}}\left(\mathbf{V}_{D}\right)$, then the linear map $\mathrm{T}_{\mathbf{v}} \mathfrak{i}$ is injective and $\mathrm{T}_{\mathbf{v}} \mathfrak{i}\left(\mathbb{T}_{\mathbf{v}}\left(\mathfrak{M}_{\mathbf{r}}\left(\mathbf{V}_{D}\right)\right)\right)=\mathbf{Z}^{(D)}(\mathbf{v})$ is linearly isomorphic to $\mathbb{T}_{\mathbf{v}}\left(\mathfrak{M}_{\mathfrak{r}}\left(\mathbf{V}_{D}\right)\right)$.

Proof. First, observe that if $\mathbf{v} \in \mathfrak{M}_{\mathfrak{r}}\left(\mathbf{V}_{D}\right)$ and $\dot{\mathbf{w}}=\mathrm{T}_{\mathbf{v}} \mathfrak{i}\left(\dot{\mathfrak{L}}, \dot{C}^{(D)}\right)$, then by Proposition 4.6(b)

$$
\dot{\mathbf{w}}=\sum_{\substack{1 \leq i_{\alpha} \leq r_{\alpha} \\ \alpha \in D}} \dot{C}_{\left(i_{\alpha}\right)_{\alpha \in D}}^{(D)} \bigotimes_{\alpha \in D} u_{i_{\alpha}}^{(\alpha)}+\sum_{\substack{1 \leq i_{\alpha} \leq r_{\alpha} \\ \alpha \in D}}\left(\dot{u}_{i_{\alpha}}^{(\alpha)} \otimes \mathbf{U}_{i_{\alpha}}^{(\alpha)}\right),
$$

where

$$
\mathbf{U}_{i_{\alpha}}^{(\alpha)}=\sum_{\substack{1 \leq i_{\beta} \leq r_{\beta} \\ \beta \in D \backslash\{\alpha\}}} C_{\left(i_{\beta}\right)_{\beta \in D}}^{(D)} \bigotimes_{\beta \in D} u_{i_{\beta}}^{(\beta)} \in U_{D \backslash\{\alpha\}}^{\min }(\mathbf{v})
$$

and $\dot{u}_{i_{\alpha}}^{(\alpha)}=\dot{L}_{\alpha}\left(u_{i_{\alpha}}^{(\alpha)}\right) \in W_{\alpha}^{\min }(\mathbf{v})$ for all $\alpha \in D$. Hence $\mathrm{T}_{\mathbf{v}} \mathfrak{i}\left(\mathbb{T}_{\mathbf{v}}\left(\mathfrak{M}_{\mathfrak{r}}\left(\mathbf{V}_{D}\right)\right)\right) \subset \mathbf{Z}^{(D)}(\mathbf{v})$. Next, we claim that $\mathbf{Z}^{(D)}(\mathbf{v}) \subset \mathbb{T}_{\mathbf{v}} \mathfrak{i}\left(\mathbb{T}_{\mathbf{v}}\left(\mathfrak{M}_{\mathfrak{r}}\left(\mathbf{V}_{D}\right)\right)\right)$. To prove the claim take $\mathbf{w} \in \mathbf{Z}^{(D)}(\mathbf{v})$. Then we can write

$$
\mathbf{w}=\sum_{\substack{1 \leq i_{\alpha} \leq r_{\alpha} \\ \alpha \in D}}\left(\dot{C}^{(D)}\right)_{\left(i_{\alpha}\right)_{\alpha \in D}} \bigotimes_{\alpha \in D} u_{i_{\alpha}}^{(\alpha)}+\sum_{\substack{1 \leq i_{\alpha} \leq r_{\alpha} \\ \alpha \in D}}\left(w_{i_{\alpha}}^{(\alpha)} \otimes \mathbf{U}_{i_{\alpha}}^{(\alpha)}\right)
$$

where $w_{i_{\alpha}}^{(\alpha)} \in W_{\alpha}^{\min }(\mathbf{v})$ for $1 \leq i_{\alpha} \leq r_{\alpha}$ and $\alpha \in D$. Now, define $\dot{L}_{\alpha} \in \mathcal{L}\left(U_{\alpha}^{\min }(\mathbf{v}), W_{\alpha}^{\min }(\mathbf{v})\right)$ by $\dot{L}_{\alpha}\left(u_{i_{\alpha}}^{(\alpha)}\right):=$ $w_{i_{\alpha}}^{(\alpha)}$ for $1 \leq i_{\alpha} \leq r_{\alpha}$ and $\alpha \in D$. Then the claim follows from $\mathbf{w}=\mathrm{T}_{\mathbf{v}} \mathfrak{i}\left(\left(\dot{L}_{\alpha}\right)_{\alpha \in D}, \dot{C}^{(D)}\right)$. To conclude the 
proof of the proposition we need to show that the map $T_{\mathbf{v}} \mathfrak{i}$ is an injective linear operator. To prove this consider that

$$
\mathrm{T}_{\mathbf{v}} \mathfrak{i}\left(\left(\dot{L}_{\beta}\right)_{\beta \in D}, \dot{C}^{(D)}\right)=\mathbf{0},
$$

that is,

$$
\mathbf{0}=\sum_{\substack{1 \leq i_{\alpha} \leq r_{\alpha} \\ \alpha \in D}}\left(\dot{C}^{(D)}\right)_{\left(i_{\alpha}\right)_{\alpha \in D}} \bigotimes_{\alpha \in D} u_{i_{\alpha}}^{(\alpha)}+\sum_{\substack{1 \leq i_{\alpha} \leq r_{\alpha} \\ \alpha \in D}} \sum_{1 \leq i_{\alpha} \leq r_{\alpha}}\left(\dot{u}_{i_{\alpha}}^{(\alpha)} \otimes \mathbf{U}_{i_{\alpha}}^{(\alpha)}\right)
$$

with $\dot{u}_{i_{\alpha}}^{(\alpha)}=\dot{L}_{\alpha}\left(u_{i_{\alpha}}^{(\alpha)}\right)$. Thus,

$$
\begin{aligned}
\sum_{\substack{1 \leq i_{\alpha} \leq r_{\alpha} \\
\alpha \in D}}\left(\dot{C}^{(D)}\right)_{\left(i_{\alpha}\right)_{\alpha \in D}} \bigotimes_{\alpha \in D} u_{i_{\alpha}}^{(\alpha)}=\mathbf{0} & \\
\sum_{1 \leq i_{\alpha} \leq r_{\alpha}}\left(\dot{u}_{i_{\alpha}}^{(\alpha)} \otimes \mathbf{U}_{i_{\alpha}}^{(\alpha)}\right) & =\mathbf{0} \text { for } \alpha \in D
\end{aligned}
$$

and hence $\dot{C}^{(D)}=\mathrm{o}$, because $\left\{\bigotimes_{\alpha \in D} u_{i_{\alpha}}^{(\alpha)}\right\}$ is a basis of ${ }_{a} \bigotimes_{\alpha \in D} U_{\alpha}^{\min }(\mathbf{v})$, and $\dot{u}_{i_{\alpha}}^{(\alpha)}=0$ for $1 \leq i_{\alpha} \leq r_{\alpha}$, because the $\left\{\mathbf{U}_{i_{\alpha}}^{(\alpha)}: 1 \leq i_{\alpha} \leq r_{\alpha}\right\}$ are linearly independent for $\alpha \in D$. Then $\dot{L}_{\alpha}=0$ for all $\alpha \in D$. We conclude that

$$
\left(\left(\dot{L}_{\beta}\right)_{\beta \in D}, \dot{C}^{(D)}\right)=\left((0)_{\beta \in D}, \mathfrak{o}\right)
$$

and, in consequence, $T_{\mathbf{v}} \mathfrak{i}$ is injective.

\subsection{The linear subspace $\mathrm{T}_{\mathbf{v}} \mathfrak{i}\left(\mathbb{T}_{\mathbf{v}}\left(\mathfrak{M}_{\mathfrak{r}}\left(\mathbf{V}_{D}\right)\right)\right)$ belongs to $\mathbb{G}\left(\mathbf{V}_{D_{\|\cdot\|_{D}}}\right)$}

Finally, to show that $\mathfrak{i}$ is an immersion, and hence $\mathfrak{M}_{\mathfrak{r}}\left(\mathbf{V}_{D}\right)$ is an immersed submanifold of $\mathbf{V}_{D_{\|\cdot\|_{D}}}$, by Proposition 4.7 we need to prove that $\mathbf{Z}^{(D)}(\mathbf{v}) \in \mathbb{G}\left(\mathbf{V}_{\|\cdot\|_{D}}\right)$. To reach it we need a slightly stronger condition than the continuity of the tensor product map. To this end we introduce the crossnorms.

\subsubsection{Crossnorms}

Let $\|\cdot\|_{\alpha}, \alpha \in D$, be the norms of the vector spaces $V_{\alpha}$ appearing in $\mathbf{V}_{D}={ }_{a} \bigotimes_{\alpha \in D} V_{\alpha}$. By $\|\cdot\|$ we denote the norm on the tensor space $\mathbf{V}_{D}$. Note that $\|\cdot\|$ is not determined by $\|\cdot\|_{\alpha}$, for $\alpha \in D$, but there are relations which are 'reasonable'. Any norm $\|\cdot\|$ on ${ }_{a} \bigotimes_{\alpha \in D} V_{\alpha}$ satisfying

$$
\left\|\bigotimes_{\alpha \in D} v_{\alpha}\right\|=\prod_{\alpha \in D}\left\|v_{\alpha}\right\|_{\alpha} \quad \text { for all } v_{\alpha} \in V_{\alpha}(\alpha \in D)
$$

is called a crossnorm. As usual, the dual norm of $\|\cdot\|$ is denoted by $\|\cdot\|^{*}$. If $\|\cdot\|$ is a crossnorm and also $\|\cdot\|^{*}$ is a crossnorm on ${ }_{a} \bigotimes_{\alpha \in D} V_{\alpha}^{*}$, i.e.,

$$
\left\|\bigotimes_{\alpha \in D} \varphi^{(\alpha)}\right\|^{*}=\prod_{\alpha \in D}\left\|\varphi^{(\alpha)}\right\|_{\alpha}^{*} \quad \text { for all } \varphi^{(\alpha)} \in V_{\alpha}^{*}(\alpha \in D),
$$

then $\|\cdot\|$ is called a reasonable crossnorm.

Remark 4.8 Eq. (4.2) implies the inequality $\left\|\bigotimes_{\alpha \in D} v_{\alpha}\right\| \lesssim \prod_{\alpha \in D}\left\|v_{\alpha}\right\|_{\alpha}$ which is equivalent to the continuity of the multilinear tensor product map (3.1).

Grothendieck [15] named the following norm $\|\cdot\|_{\vee}$ the injective norm.

Definition 4.9 Let $V_{\alpha}$ be a Banach space with norm $\|\cdot\|_{\alpha}$ for $\alpha \in D$. Then for $\mathbf{v} \in \mathbf{V}={ }_{a} \bigotimes_{\alpha \in D} V_{\alpha}$ define $\|\cdot\|_{\vee\left(V_{1}, \ldots, V_{d}\right)}$ by

$$
\|\mathbf{v}\|_{\vee\left(V_{1}, \ldots, V_{d}\right)}:=\sup \left\{\frac{\left|\left(\varphi_{1} \otimes \varphi_{2} \otimes \ldots \otimes \varphi_{d}\right)(\mathbf{v})\right|}{\prod_{\alpha \in D}\left\|\varphi_{\alpha}\right\|_{\alpha}^{*}}: 0 \neq \varphi_{\alpha} \in V_{\alpha}^{*}, \alpha \in D\right\} .
$$


It is well known that the injective norm is a reasonable crossnorm (see Lemma 1.6 in [26] and (4.2)-(4.3)). Further properties are given by the next proposition (see Lemma 4.96 and Section 4.2 .4 in [19]).

Proposition 4.10 Let $V_{\alpha}$ be a Banach space with norm $\|\cdot\|_{\alpha}$ for $\alpha \in D$, and $\|\cdot\|$ be a norm on $\mathbf{V}_{D}=$ ${ }_{a} \bigotimes_{\alpha \in D} V_{\alpha}$. The following statements hold.

(a) For each $\alpha \in D$ introduce the tensor Banach space $\mathbf{X}_{\alpha}:=\|\cdot\|_{\vee\left(V_{1}, \ldots, V_{\alpha-1}, V_{\alpha+1}, \ldots, V_{d}\right)} \bigotimes_{\beta \neq \alpha} V_{\beta}$. Then

$$
\|\cdot\|_{\vee}\left(V_{1}, \ldots, V_{d}\right)=\|\cdot\|_{\vee}\left(V_{\alpha}, \mathbf{X}_{\alpha}\right)
$$

holds for $\alpha \in D$.

(b) The injective norm is the weakest reasonable crossnorm on $\mathbf{V}$, i.e., if $\|\cdot\|$ is a reasonable crossnorm on $\mathbf{V}$, then

$$
\|\cdot\| \gtrsim\|\cdot\|_{\vee\left(V_{1}, \ldots, V_{d}\right)}
$$

(c) For any norm $\|\cdot\|$ on $\mathbf{V}$ satisfying $\|\cdot\|_{\vee\left(V_{1}, \ldots, V_{d}\right)} \lesssim\|\cdot\|$, the map (3.1) is continuous, and hence Fréchet differentiable.

The following result shows an interesting use of the injective norm.

Corollary 4.11 Assume that $\left(V_{\alpha},\|\cdot\|_{\alpha}\right)$ is a normed space for each $\alpha \in D$. Then the algebraic tensor space $\mathbf{V}={ }_{a} \bigotimes_{\alpha \in D} V_{\alpha}$ is a $\mathcal{C}^{\infty}$-Banach manifold not modelled on a particular Banach space.

Proof. Let $V_{\alpha_{\|\cdot\|_{\alpha}}}$ be the Banach space obtained by the completion of $V_{\alpha}$ by using the norm $\|\cdot\|_{\alpha}$ for $\alpha \in D$. Then we have

$$
\mathbf{V}={ }_{a} \bigotimes_{\alpha \in D} V_{\alpha} \subset \mathbf{V}^{\star}={ }_{a} \bigotimes_{\alpha \in D} V_{\alpha_{\|\cdot\|_{\alpha}}}
$$

From Proposition 4.10(c) the map

$$
\bigotimes:\left(\underset{\alpha \in D}{X} V_{\alpha_{\|\cdot\|_{\alpha}}},\|\cdot\|\right) \longrightarrow\left(\mathbf{V}^{\star},\|\cdot\|_{\vee\left(V_{\|_{\|\cdot\|_{1}}}, \ldots, V_{d_{\|\cdot\|_{d}}}\right)}\right)
$$

is continuous and hence

$$
\bigotimes:\left(\underset{\alpha \in D}{X} V_{\alpha},\|\cdot\|\right) \longrightarrow\left(\mathbf{V},\|\cdot\|_{\vee\left(V_{1_{\|\cdot\|_{1}}}, \ldots, V_{d_{\|\cdot\|_{d}}}\right)}\right)
$$

is also continuous. Then Corollary 3.22 proves the desired conclusion.

Remark 4.12 Observe that from the proof of the above corollary, we can conclude that $\mathbf{V}^{\star}={ }_{a} \bigotimes_{\alpha \in D} V_{\alpha_{\|\cdot\| \alpha}}$ is also a $\mathcal{C}^{\infty}$-Banach manifold not modelled on a particular Banach space.

\subsection{2 $\mathrm{T}_{\mathbf{v}} \mathfrak{i}\left(\mathbb{T}_{\mathbf{v}}\left(\mathfrak{M}_{\mathfrak{r}}\left(\mathbf{V}_{D}\right)\right)\right)$ belongs to $\mathbb{G}\left(\mathbf{V}_{D_{\|\cdot\|_{D}}}\right)$}

We will assume that the norm $\|\cdot\|_{D}$ on $\mathbf{V}_{D}$ satisfies

$$
\|\cdot\|_{\vee\left(V_{1}, \ldots, V_{d}\right)} \lesssim\|\cdot\|_{D}
$$

and hence, by Proposition 4.10(c), under this condition, Proposition 4.7 also holds. A first useful result is the following lemma.

Lemma 4.13 Assume that $\left(V_{\alpha},\|\cdot\|_{\alpha}\right)$ is a normed space for each $\alpha \in D$ and let $\|\cdot\|_{D}$ be a norm on the tensor space $\mathbf{V}_{D}={ }_{a} \bigotimes_{\alpha \in D} V_{\alpha}$ such that (4.7) holds. Let $\beta \in D$. If $W_{\beta} \in \mathbb{G}\left(V_{\beta_{\|\cdot\|_{\beta}}}\right)$ satisfies $V_{\beta_{\|\cdot\|_{\beta}}}=U_{\beta} \oplus W_{\beta}$ for some finite-dimensional subspace $U_{\beta}$ in $V_{\beta_{\|\cdot\|_{\beta}}}$, then $W_{\beta} \otimes_{a} U_{[\beta]} \in \mathbb{G}\left(\mathbf{V}_{D_{\|\cdot\|_{D}}}\right)$ for every finite-dimensional subspace $U_{[\beta]} \subset \mathbf{V}_{[\beta]}={ }_{a} \bigotimes_{\delta \in D \backslash\{\beta\}} V_{\delta_{\|\cdot\|_{\delta}}}$. 
Proof. First, observe that if $W_{\beta}$ is a finite-dimensional subspace, then $W_{\beta} \otimes_{a} U_{[\beta]}$ is also finite-dimensional, and hence the lemma follows. Thus, assume that $W_{\beta}$ is an infinite-dimensional closed subspace of $V_{\beta_{\|\cdot\|_{\beta}}}$, and to simplify the notation write

$$
\mathbf{X}_{\beta}:=\|\cdot\|_{\vee\left(V_{1}, \ldots, V_{\beta-1}, V_{\beta+1}, \ldots, V_{d}\right)} \bigotimes_{\delta \in D \backslash\{\beta\}} \mathbf{V}_{\delta_{\|\cdot\|_{\delta}}}
$$

If $U_{[\beta]} \subset \mathbf{X}_{\beta}$ is a finite-dimensional subspace, then there exists $W_{[\beta]} \in \mathbb{G}\left(\mathbf{X}_{\beta}\right)$ such that $\mathbf{X}_{\beta}=U_{[\beta]} \oplus W_{[\beta]}$. Since the tensor product map

$$
\bigotimes:\left(V_{\beta_{\|\cdot\|_{\beta}}},\|\cdot\|_{\beta}\right) \times\left(\mathbf{X}_{\beta},\|\cdot\|_{\vee\left(V_{1}, \ldots, V_{\beta-1}, V_{\beta+1}, \ldots, V_{d}\right)}\right) \rightarrow\left(\mathbf{V}_{D_{\|\cdot\|_{D}}},\|\cdot\|_{D}\right)
$$

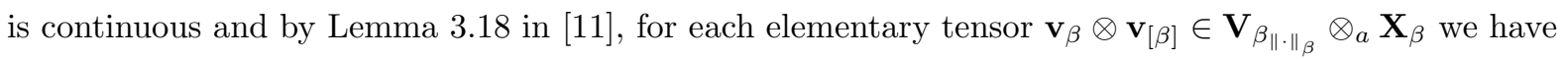

$$
\begin{aligned}
\left\|\left(i d_{\beta} \otimes P_{U_{[\beta]} \oplus W_{[\beta]}}\right)\left(\mathbf{v}_{\beta} \otimes \mathbf{v}_{[\beta]}\right)\right\|_{\alpha} & \leq C \sqrt{\operatorname{dim} U_{[\beta]}}\left\|\mathbf{v}_{\beta}\right\|_{\beta}\left\|\mathbf{v}_{[\beta]}\right\|_{\vee}\left(V_{1}, \ldots, V_{\beta-1}, V_{\beta+1}, \ldots, V_{d}\right) \\
& =C \sqrt{\operatorname{dim} U_{[\beta]}}\left\|\mathbf{v}_{\beta} \otimes \mathbf{v}_{[\beta]}\right\|_{\vee}\left(V_{1}, \ldots, V_{\beta-1}, V_{\beta}, V_{\beta+1}, \ldots, V_{d}\right) \\
& \leq C^{\prime} \sqrt{\operatorname{dim} U_{[\beta]}}\left\|\mathbf{v}_{\beta} \otimes \mathbf{v}_{[\beta]}\right\|_{D} .
\end{aligned}
$$

Thus, $\left(i d_{\beta} \otimes P_{U_{[\beta]} \oplus W_{[\beta]}}\right)$ is continuous over $\mathbf{V}_{\beta_{\|\cdot\|_{\beta}}} \otimes_{a} \mathbf{X}_{\beta}$, and hence in $\mathbf{V}_{D_{\|\cdot\|_{D}}}$. Now, take into account the fact that

$$
i d_{\beta}=P_{U_{\beta} \oplus W_{\beta}}+P_{W_{\beta} \oplus U_{\beta}}
$$

so that

$$
i d_{\beta} \otimes P_{U_{[\beta]} \oplus W_{[\beta]}}=P_{U_{\beta} \oplus W_{\beta}} \otimes P_{U_{[\beta]} \oplus W_{[\beta]}}+P_{W_{\beta} \oplus U_{\beta}} \otimes P_{U_{[\beta]} \oplus W_{[\beta]}} .
$$

Observe that $i d_{\beta} \otimes P_{U_{[\beta]} \oplus W_{[\beta]}}$ and $P_{U_{\beta} \oplus W_{\beta}} \otimes P_{U_{[\beta]} \oplus W_{[\beta]}}$ are continuous linear maps over $\mathbf{V}_{\beta_{\|\cdot\|_{\beta}}} \otimes_{a} \mathbf{X}_{\beta}$, and then $P_{W_{\beta} \oplus U_{\beta}} \otimes P_{U_{[\beta]} \oplus W_{[\beta]}}$ is a continuous linear map over $\mathbf{V}_{\beta_{\|\cdot\|_{\beta}}} \otimes_{a} \mathbf{X}_{\beta}$. Thus,

$$
\mathcal{P}:=\overline{P_{W_{\beta} \oplus U_{\beta}} \otimes P_{U_{[\beta]} \oplus W_{[\beta]}}} \in \mathcal{L}\left(\mathbf{V}_{D_{\|\cdot\|_{D}}}, \mathbf{V}_{D_{\|\cdot\|_{D}}}\right)
$$

and $\mathcal{P} \circ \mathcal{P}=\mathcal{P}$. Since $\mathcal{P}\left(\mathbf{V}_{D_{\|\cdot\|_{D}}}\right)=W_{\beta} \otimes_{a} U_{[\beta]}$, the lemma follows by Proposition 2.8 .

Lemma 4.14 Let $X$ be a Banach space and assume that $U, V \in \mathbb{G}(X)$. If $U \cap V=\{0\}$, then $U \oplus V \in \mathbb{G}(X)$. Moreover, $U \cap V \in \mathbb{G}(X)$ holds.

Proof. To prove the first statement assume that $U \cap V=\{0\}$. Since $U, V \in \mathbb{G}(X)$ there exist $U^{\prime}, V^{\prime} \in \mathbb{G}(X)$, such that $X=U \oplus U^{\prime}=V \oplus V^{\prime}$. Then $U=X \cap U=\left(V \oplus V^{\prime}\right) \cap U=U \cap V^{\prime}$ and $V=X \cap V=\left(U \oplus U^{\prime}\right) \cap V=$ $V \cap U^{\prime}$. In consequence, we can write

$$
U \oplus V \oplus\left(U^{\prime} \cap V^{\prime}\right)=\left(U \cap V^{\prime}\right) \oplus\left(V \cap U^{\prime}\right) \oplus\left(U^{\prime} \cap V^{\prime}\right)=\left(U \oplus U^{\prime}\right) \cap\left(V \oplus V^{\prime}\right)=X,
$$

and the first statement follows. To prove the second one, observe that $X=(U \cap V) \oplus\left(U \cap V^{\prime}\right) \oplus\left(V \cap U^{\prime}\right) \oplus$ $\left(U^{\prime} \cap V^{\prime}\right)$.

An important consequence of the above two lemmas is the following theorem.

Theorem 4.15 Assume that $\left(V_{\alpha},\|\cdot\|_{\alpha}\right)$ is a normed space for each $\alpha \in D$ and let $\|\cdot\|_{D}$ be a norm on the tensor space $\mathbf{V}_{D}={ }_{a} \bigotimes_{\alpha \in D} V_{\alpha}$ such that (4.7) holds. Then for each $\mathbf{v} \in \mathfrak{M}_{\mathfrak{r}}\left(\mathbf{V}_{D}\right)$ we have $\mathbf{Z}^{(D)}(\mathbf{v}) \in$ $\mathbb{G}\left(\mathbf{V}_{D_{\|\cdot\|_{D}}}\right)$, and hence $\mathfrak{M}_{\mathfrak{r}}\left(\mathbf{V}_{D}\right)$ is an immersed submanifold of $\mathbf{V}_{D_{\|\cdot\|_{D}}}$.

Proof. For each $\alpha \in D$ we have $W_{\alpha}^{\min }(\mathbf{v}) \in \mathbb{G}\left(V_{\alpha_{\|\cdot\|_{\alpha}}}\right)$ and $U_{D \backslash\{\alpha\}}^{\min }(\mathbf{v}) \subset{ }_{a} \bigotimes_{\delta \in D \backslash\{\alpha\}} \mathbf{V}_{\delta_{\|\cdot\|_{\delta}}}$ is a finitedimensional subspace. From Lemma 4.13 we have $W_{\alpha}^{\min }(\mathbf{v}) \otimes_{a} U_{D \backslash\{\alpha\}}^{\min }(\mathbf{v}) \in \mathbb{G}\left(\mathbf{V}_{D_{\|\cdot\|_{D}}}\right)$ for all $\alpha \in D$. Since ${ }_{a} \bigotimes_{\alpha \in D} U_{\alpha}^{\min }(\mathbf{v}) \in \mathbb{G}\left(\mathbf{V}_{D_{\|\cdot\|_{D}}}\right)$, by Lemma 4.14, we obtain that $\mathbf{Z}^{(D)}(\mathbf{v}) \in \mathbb{G}\left(\mathbf{V}_{D_{\|\cdot\|_{D}}}\right)$. 
Example 4.16 Let us recall the topological tensor spaces introduced in Example 3.3. Let $I_{\alpha} \subset \mathbb{R}(\alpha \in D)$ and $1 \leq p<\infty$. Let $\mathbf{I}:=\times_{\alpha \in D} I_{\alpha}$. Hence $L^{p}(\mathbf{I})$ is a tensor Banach space for all $\alpha \in T_{D}$. In this example we denote the usual norm of $L^{p}(\mathbf{I})$ by $\|\cdot\|_{0, p}$. Since $\|\cdot\|_{0, p}$ is a reasonable crossnorm (see Example 4.72 in [19]), then (4.7) holds. From Theorem 4.15 we obtain that $\mathfrak{M}_{\mathfrak{r}}\left({ }_{a} \bigotimes_{\alpha \in D} L^{p}\left(I_{\alpha}\right)\right)$ is an immersed submanifold of $L^{p}(\mathbf{I})$.

Example 4.17 Now, we return to Example [3.20. From Example 4.42 in [19] we know that the norm $\|\cdot\|_{(0,1), p}$ is a crossnorm on $H^{1, p}\left(I_{1}\right) \otimes_{a} H^{1, p}\left(I_{2}\right)$, and hence it is not weaker than the injective norm. In consequence, from Theorem 4.15, we obtain that $\mathfrak{M}_{\mathfrak{r}}\left(H^{1, p}\left(I_{1}\right) \otimes_{a} H^{1, p}\left(I_{2}\right)\right)$ is an immersed submanifold in $H^{1, p}\left(I_{1}\right) \otimes_{\|\cdot\|_{(0,1), p}} H^{1, p}\left(I_{2}\right)$.

Since in a reflexive Banach space every closed linear subspace is proximinal (see p. 61 in [13]), we have the following corollary.

Corollary 4.18 Assume that $\left(V_{\alpha},\|\cdot\|_{\alpha}\right)$ is a normed space for each $\alpha \in D$ and let $\|\cdot\|_{D}$ be a norm on the

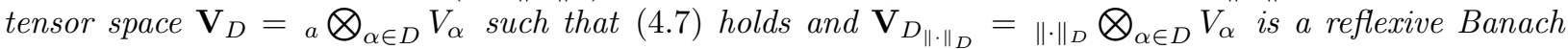
space. Then for any $\mathbf{v} \in \mathfrak{M}_{\mathfrak{r}}\left(\mathbf{V}_{D}\right)$ and $\dot{\mathbf{u}} \in \mathbf{V}_{D_{\|\cdot\|_{D}}}$, there exists $\dot{\mathbf{v}}_{\text {best }} \in \mathbf{Z}^{(D)}(\mathbf{v})$ such that

$$
\left\|\dot{\mathbf{u}}-\dot{\mathbf{v}}_{\text {best }}\right\|=\min _{\dot{\mathbf{v}} \in \mathbf{Z}^{(D)}(\mathbf{v})}\|\dot{\mathbf{u}}-\dot{\mathbf{v}}\| .
$$

\section{On the Dirac-Frenkel variational principle on tensor Banach spaces}

\subsection{Model reduction in tensor Banach spaces}

In this section we consider the abstract ordinary differential equation in a reflexive tensor Banach space $\mathbf{V}_{D_{\|\cdot\|_{D}}}$, given by

$$
\begin{aligned}
\dot{\mathbf{u}}(t) & =\mathbf{F}(t, \mathbf{u}(t)), \text { for } t \geq 0 \\
\mathbf{u}(0) & =\mathbf{u}_{0},
\end{aligned}
$$

where we assume $\mathbf{u}_{0} \neq \mathbf{0}$ and $\mathbf{F}:[0, \infty) \times \mathbf{V}_{D_{\|\cdot\|_{D}}} \longrightarrow \mathbf{V}_{D_{\|\cdot\|_{D}}}$ satisfying the usual conditions to have existence and uniqueness of solutions. As usual we assume that $\left(V_{\alpha},\|\cdot\|_{\alpha}\right)$ is a normed space for each $\alpha \in D$ and let $\|\cdot\|_{D}$ be a norm on the tensor space $\mathbf{V}_{D}={ }_{a} \bigotimes_{\alpha \in D} V_{\alpha}$ such that (4.7) holds. We want to approximate $\mathbf{u}(t)$, for $t \in I:=(0, T)$ for some $T>0$, by a differentiable curve $t \mapsto \mathbf{v}_{r}(t)$ from $I$ to $\mathfrak{M}_{\mathfrak{r}}\left(\mathbf{V}_{D}\right)$, where $\mathfrak{r} \in \mathcal{A D}\left(\mathbf{V}_{D}\right)(\mathfrak{r} \neq \mathbf{0})$, such that $\mathbf{v}_{r}(0)=\mathbf{v}_{0} \in \mathfrak{M}_{\mathfrak{r}}\left(\mathbf{V}_{D}\right)$ is an approximation of $\mathbf{u}_{0} 3$

Our main goal is to construct a reduced order model of (5.1) -(5.2) over the Banach manifold $\mathfrak{M}_{\mathfrak{r}}\left(\mathbf{V}_{D}\right)$. Since $\mathbf{F}\left(t, \mathbf{v}_{r}(t)\right) \in \mathbf{V}_{D_{\|\cdot\|_{D}}}$, for each $t \in I$, and $\mathbf{Z}^{(D)}\left(\mathbf{v}_{r}(t)\right)$ is a closed linear subspace in $\mathbf{V}_{D_{\|\cdot\|_{D}}}$, we have the existence of a $\dot{\mathbf{v}}_{r}(t) \in \mathbf{Z}^{(D)}\left(\mathbf{v}_{r}(t)\right)$ such that

$$
\left\|\dot{\mathbf{v}}_{r}(t)-\mathbf{F}\left(t, \mathbf{v}_{r}(t)\right)\right\|_{D}=\min _{\dot{\mathbf{v}} \in \mathbf{Z}^{(D)}\left(\mathbf{v}_{r}(t)\right)}\left\|\dot{\mathbf{v}}-\mathbf{F}\left(t, \mathbf{v}_{r}(t)\right)\right\|_{D} .
$$

It is well known that, if $\mathbf{V}_{D_{\|\cdot\|_{D}}}$ is a Hilbert space, then $\dot{\mathbf{v}}_{r}(t)=\mathcal{P}_{\mathbf{v}_{r}(t)}\left(\mathbf{F}\left(t, \mathbf{v}_{r}(t)\right)\right)$, where

$$
\mathcal{P}_{\mathbf{v}_{r}(t)}=\mathcal{P}_{\mathbf{Z}^{(D)}\left(\mathbf{v}_{r}(t)\right) \oplus \mathbf{Z}^{(D)}\left(\mathbf{v}_{r}(t)\right)^{\perp}}
$$

is called the metric projection. It has the following important property: $\dot{\mathbf{v}}_{r}(t)=\mathcal{P}_{\mathbf{v}_{r}(t)}\left(\mathbf{F}\left(t, \mathbf{v}_{r}(t)\right)\right)$ if and only if

$$
\left\langle\dot{\mathbf{v}}_{r}(t)-\mathbf{F}\left(t, \mathbf{v}_{r}(t)\right), \dot{\mathbf{v}}\right\rangle_{D}=0 \text { for all } \dot{\mathbf{v}} \in \mathbf{Z}^{(D)}\left(\mathbf{v}_{r}(t)\right) .
$$

The concept of a metric projection can be extended to the Banach space setting. To this end we recall the following definitions. A Banach space $X$ with norm $\|\cdot\|$ is said to be strictly convex if $\|x+y\| / 2<1$

\footnotetext{
${ }^{3} \mathbf{v}_{0}$ can be chosen as the best approximation of $\mathbf{u}_{0}$ in $\mathfrak{M}_{\mathfrak{r}}\left(\mathbf{V}_{D}\right)$ because a best approximation exists [11].
} 
for all $x, y \in X$ with $\|x\|=\|y\|=1$ and $x \neq y$. It is uniformly convex if $\lim _{n \rightarrow \infty}\left\|x_{n}-y_{n}\right\|=0$ for any two sequences $\left\{x_{n}\right\}_{n \in \mathbb{N}}$ and $\left\{y_{n}\right\}_{n \in \mathbb{N}}$ such that $\left\|x_{n}\right\|=\left\|y_{n}\right\|=1$ and $\lim _{n \rightarrow \infty}\left\|x_{n}+y_{n}\right\| / 2=1$. It is known that a uniformly convex Banach space is reflexive and strictly convex. A Banach space $X$ is said to be smooth if the limit

$$
\lim _{t \rightarrow 0} \frac{\|x+t y\|-\|x\|}{t}
$$

exists for all $x, y \in \mathbb{S}_{X}=\{z \in X:\|z\|=1\}$. Finally, a Banach space $X$ is said to be uniformly smooth if its modulus of smoothness

$$
\rho(\tau)=\sup _{x, y \in \mathbb{S}_{X}}\left\{\frac{\|x+\tau y\|+\|x-\tau y\|}{2}-1\right\}, \tau>0,
$$

satisfies the condition $\lim _{\tau \rightarrow 0} \rho(\tau)=0$. In uniformly smooth spaces, and only in such spaces, the norm is uniformly Fréchet differentiable. A uniformly smooth Banach space is smooth. The converse is true if the Banach space is finite-dimensional. It is known that the space $L^{p}(1<p<\infty)$ is a uniformly convex and uniformly smooth Banach space.

Let $\langle\cdot, \cdot\rangle: X \times X^{*} \longrightarrow \mathbb{R}$ denote the duality pairing, i.e.,

$$
\langle x, f\rangle:=f(x) .
$$

The normalised duality mapping $J: X \longrightarrow 2^{X^{*}}$ is defined by

$$
J(x):=\left\{f \in X^{*}:\langle x, f\rangle=\|x\|^{2}=\left(\|f\|^{*}\right)^{2}\right\},
$$

and it has the following properties (see [2]):

(a) If $X$ is smooth, the map $J$ is single-valued;

(b) if $X$ is smooth, then $J$ is norm-to-weak* continuous;

(c) if $X$ is uniformly smooth, then $J$ is uniformly norm-to-norm continuous on each bounded subset of $X$.

Remark 5.1 In a Hilbert space, the normalised duality mapping is the Riesz map. Notice that after identifying $X$ with $X^{*}$, it can be shown (see Proposition 4.8(i) in [7]) that the normalised duality mapping is the identity operator.

Assume that $\left(V_{\alpha},\|\cdot\|_{\alpha}\right)$ is a normed space for each $\alpha \in D$. Let $\mathbf{V}_{D_{\|\cdot\|_{D}}}=\|\cdot\|_{D} \otimes_{\alpha \in D} V_{\alpha}$ be a reflexive and strictly convex tensor Banach space such that (4.7) holds. For $\mathbf{F}\left(t, \mathbf{v}_{r}(t)\right)$ in $\mathbf{V}_{D_{\|\cdot\|_{D}}}$, with a fixed $t \in I$, it is known that the set

$$
\left\{\dot{\mathbf{v}}_{r}(t):\left\|\dot{\mathbf{v}}_{r}(t)-\mathbf{F}\left(t, \mathbf{v}_{r}(t)\right)\right\|_{D}=\min _{\dot{\mathbf{v}} \in \mathbf{Z}^{(D)}\left(\mathbf{v}_{r}(t)\right)}\left\|\dot{\mathbf{v}}-\mathbf{F}\left(t, \mathbf{v}_{r}(t)\right)\right\|_{D}\right\}
$$

is always a singleton. Let $\mathcal{P}_{\mathbf{v}_{r}(t)}$ be the mapping from $\mathbf{V}_{D_{\|\cdot\|_{D}}}$ onto $\mathbf{Z}^{(D)}\left(\mathbf{v}_{r}(t)\right)$ defined by $\dot{\mathbf{v}}_{r}(t):=$ $\mathcal{P}_{\mathbf{v}_{r}(t)}\left(\mathbf{F}\left(t, \mathbf{v}_{r}(t)\right)\right)$ if and only if

$$
\left\|\dot{\mathbf{v}}_{r}(t)-\mathbf{F}\left(t, \mathbf{v}_{r}(t)\right)\right\|_{D}=\min _{\dot{\mathbf{v}} \in \mathbf{Z}^{(D)}\left(\mathbf{v}_{r}(t)\right)}\left\|\dot{\mathbf{v}}-\mathbf{F}\left(t, \mathbf{v}_{r}(t)\right)\right\|_{D} .
$$

It is also called the metric projection. The classical characterisation of the metric projection together with Proposition 2.10 of [2] allows us to state the next result.

Theorem 5.2 Assume that $\left(V_{\alpha},\|\cdot\|_{\alpha}\right)$ is a normed space for each $\alpha \in D$. Let $\mathbf{V}_{D_{\|\cdot\|_{D}}}=\|\cdot\|_{D} \bigotimes_{\alpha \in D} V_{\alpha}$ be a reflexive and strictly convex tensor Banach space such that (4.7) holds. Then for each $t \in I$ the following statements are equivalent.

(a) $\dot{\mathbf{v}}_{r}(t)=\mathcal{P}_{\mathbf{v}_{r}(t)}\left(\mathbf{F}\left(t, \mathbf{v}_{r}(t)\right)\right)$. 
(b) $\left\langle\dot{\mathbf{v}}_{r}(t)-\dot{\mathbf{v}}, J\left(\mathbf{F}\left(t, \mathbf{v}_{r}(t)\right)-\dot{\mathbf{v}}_{r}(t)\right)\right\rangle \geq 0$ for all $\dot{\mathbf{v}} \in \mathbf{Z}^{(D)}\left(\mathbf{v}_{r}(t)\right)$.

(c) $\left\langle\dot{\mathbf{v}}, J\left(\mathbf{F}\left(t, \mathbf{v}_{r}(t)\right)-\dot{\mathbf{v}}_{r}(t)\right)\right\rangle=0$ for all $\dot{\mathbf{v}} \in \mathbf{Z}^{(D)}\left(\mathbf{v}_{r}(t)\right)$.

An alternative approach is the use of the so-called generalised projection operator (see also [2]). To formulate this, we will use the following framework. Let $\mathbf{V}_{D_{\|\cdot\|_{D}}}$ be a reflexive, strictly convex and smooth tensor Banach space. Following [23, we can define a function $\phi: \mathbf{V}_{D_{\|\cdot\|_{D}}} \times \mathbf{V}_{D_{\|\cdot\|_{D}}} \longrightarrow \mathbb{R}$ by

$$
\phi(\mathbf{u}, \mathbf{v})=\|\mathbf{u}\|_{D}^{2}-2\langle\mathbf{u}, J(\mathbf{v})\rangle+\|\mathbf{v}\|_{D}^{2},
$$

where $\langle\cdot, \cdot\rangle$ denotes the duality pairing and $J$ is the normalised duality mapping. It is known that the set

$$
\left\{\dot{\mathbf{v}}_{r}(t): \phi\left(\dot{\mathbf{v}}_{r}(t), \mathbf{F}\left(t, \mathbf{v}_{r}(t)\right)\right)=\min _{\dot{\mathbf{v}} \in \mathbf{Z}^{(D)}\left(\mathbf{v}_{r}(t)\right)} \phi\left(\dot{\mathbf{v}}, \mathbf{F}\left(t, \mathbf{v}_{r}(t)\right)\right)\right\}
$$

is always a singleton. It allows us to define a map $\Pi_{\mathbf{v}_{r}(t)}: \mathbf{V}_{D_{\|\cdot\|_{D}}} \longrightarrow \mathbf{Z}^{(D)}\left(\mathbf{v}_{r}(t)\right)$ by $\dot{\mathbf{v}}_{r}(t):=\Pi_{\mathbf{v}_{r}(t)}\left(\mathbf{F}\left(t, \mathbf{v}_{r}(t)\right)\right)$ if and only if

$$
\phi\left(\dot{\mathbf{v}}_{r}(t), \mathbf{F}\left(t, \mathbf{v}_{r}(t)\right)\right)=\min _{\dot{\mathbf{v}} \in \mathbf{Z}^{(D)}\left(\mathbf{v}_{r}(t)\right)} \phi\left(\dot{\mathbf{v}}, \mathbf{F}\left(t, \mathbf{v}_{r}(t)\right)\right)
$$

The map $\Pi_{\mathbf{v}_{r}(t)}$ is called the generalised projection. It coincides with the metric projection when $\mathbf{V}_{D_{\|\cdot\|_{D}}}$ is a Hilbert space.

Remark 5.3 We point out that, in general, the operators $\mathcal{P}_{\mathbf{v}_{r}(t)}$ and $\Pi_{\mathbf{v}_{r}(t)}$ are nonlinear in Banach (not Hilbert) spaces.

Again, a classical characterisation of the generalised projection gives us the following theorem.

Theorem 5.4 Assume that $\left(V_{\alpha},\|\cdot\|_{\alpha}\right)$ is a normed space for each $\alpha \in D$. Let $\mathbf{V}_{D_{\|\cdot\|_{D}}=\|\cdot\|_{D}} \bigotimes_{\alpha \in D} V_{\alpha}$ be a reflexive and strictly convex tensor Banach space such that (4.7) holds. Then for each $t \in I$ we have

$$
\dot{\mathbf{v}}_{r}(t)=\Pi_{\mathbf{v}_{r}(t)}\left(\mathbf{F}\left(t, \mathbf{v}_{r}(t)\right)\right)
$$

if and only if

$$
\left\langle\dot{\mathbf{v}}_{r}(t)-\dot{\mathbf{v}}, J\left(\mathbf{F}\left(t, \mathbf{v}_{r}(t)\right)\right)-J\left(\dot{\mathbf{v}}_{r}(t)\right)\right\rangle \geq 0 \text { for all } \dot{\mathbf{v}} \in \mathbf{Z}^{(D)}\left(\mathbf{v}_{r}(t)\right)
$$

\subsection{The time--dependent Hartree method}

Assume that $\left(V_{\alpha},\|\cdot\|_{\alpha}\right)$ is a Banach space for each $\alpha \in D$. Let $\mathbf{V}_{\|\cdot\|}=\|\cdot\|_{D} \bigotimes_{\alpha \in D} V_{\alpha}$ be a reflexive and strictly convex tensor Banach space such that (4.7) holds. Let us consider in $\mathbf{V}_{\|\cdot\|}$ a flow generated by a densely defined operator $A \in L\left(\mathbf{V}_{\|\cdot\|}, \mathbf{V}_{\|\cdot\|}\right)$. More precisely, there exists a collection of bijective maps $\varphi_{t}: \mathcal{D}(A) \longrightarrow \mathcal{D}(A)$, here $\mathcal{D}(A)$ denotes the domain of $A$, satisfying

(i) $\varphi_{0}=\mathbf{i d}$,

(ii) $\varphi_{t+s}=\varphi_{t} \circ \varphi_{s}$, and

(iii) for $\mathbf{u}_{0} \in \mathcal{D}(A)$, the map $t \mapsto \varphi_{t}$ is differentiable as a curve in $\mathbf{V}_{\|\cdot\|}$, and $\mathbf{u}(t):=\boldsymbol{\varphi}_{t}\left(\mathbf{u}_{0}\right)$ satisfies

$$
\begin{aligned}
\dot{\mathbf{u}} & =A \mathbf{u}, \\
\mathbf{u}(0) & =\mathbf{u}_{0} .
\end{aligned}
$$

In this framework we want to study the approximation of a solution $\mathbf{u}(t)=\boldsymbol{\varphi}_{t}\left(\mathbf{u}_{0}\right) \in \mathbf{V}_{\|\cdot\|}$ by a curve $\mathbf{v}_{r}(t):=\lambda(t) \otimes_{\alpha \in D} v_{\alpha}(t)$ in the Banach manifold $\mathfrak{M}_{(1, \ldots, 1)}(\mathbf{V})$, also called in [27] the Hartree manifold. The time-dependent Hartree method consists in the use of the Dirac-Frenkel variational principle on the Hartree manifold. More precisely, we want to solve the following reduced order model:

$$
\begin{aligned}
\dot{\mathbf{v}}_{r}(t) & =\mathcal{P}_{\mathbf{v}_{r}(t)}\left(A \mathbf{v}_{r}(t)\right) \text { for } t \in I, \\
\mathbf{v}_{r}(0) & =\mathbf{v}_{0},
\end{aligned}
$$


with $\mathbf{v}_{0}=\lambda_{0} \otimes_{\alpha \in D} v_{0}^{(\alpha)} \in \mathfrak{M}_{(1, \ldots, 1)}(\mathbf{V})$ being an approximation of $\mathbf{u}_{0}$. By using the characterisation of the metric projection in a Banach space, for each $t>0$ we would like to find $\dot{\mathbf{v}}_{r}(t) \in \mathrm{T}_{\mathbf{v}_{r}(t)} \mathfrak{i}\left(\mathbb{T}_{\mathbf{v}_{r}(t)}\left(\mathfrak{M}_{(1, \ldots, 1)}(\mathbf{V})\right)\right)$ such that

$$
\begin{gathered}
\left\langle\dot{\mathbf{v}}, J\left(\dot{\mathbf{v}}_{r}(t)-A \mathbf{v}_{r}(t)\right)\right\rangle=0 \text { for all } \dot{\mathbf{v}} \in \mathrm{T}_{\mathbf{v}_{r}(t)} \mathfrak{i}\left(\mathbb{T}_{\mathbf{v}_{r}(t)}\left(\mathfrak{M}_{(1, \ldots, 1)}(\mathbf{V})\right)\right), \\
\mathbf{v}_{r}(0)=\mathbf{v}_{0}=\lambda_{0} \otimes_{\alpha \in D} v_{0}^{(\alpha)}
\end{gathered}
$$

A first result is the following Lemma.

Lemma 5.5 Let $\mathbf{v} \in \mathcal{C}^{1}\left(I, \mathcal{U}\left(\mathbf{v}_{0}\right)\right)$, where $\mathbf{v}(0)=\mathbf{v}_{0} \in \mathfrak{M}_{(1, \ldots, 1)}(\mathbf{V})$ and $\left(\mathcal{U}\left(\mathbf{v}_{0}\right), \Theta_{\mathbf{v}_{0}}\right)$ is a local chart at $\mathbf{v}_{0}$ in $\mathfrak{M}_{(1, \ldots, 1)}(\mathbf{V})$. Assume that $\mathbf{v}$ is also a $\mathcal{C}^{1}$-morphism between the manifolds $I \subset \mathbb{R}$ and $\mathcal{U}\left(\mathbf{v}_{0}\right) \subset \mathfrak{M}_{(1, \ldots, 1)}(\mathbf{V})$ such that $\mathbf{v}(t)=\lambda(t) \bigotimes_{\alpha \in D} v_{\alpha}(t)$ for some $\lambda \in \mathcal{C}^{1}(I, \mathbb{R})$ and $v_{\alpha} \in \mathcal{C}^{1}\left(I, V_{\alpha}\right)$ for $\alpha \in D$. Then

$$
\dot{\mathbf{v}}(t)=\dot{\lambda}(t) \bigotimes_{\alpha \in D} v_{\alpha}(t)+\lambda(t) \sum_{\alpha \in D} \dot{v}_{\alpha}(t) \otimes \bigotimes_{\substack{\beta \in D \\ \beta \neq \alpha}} v_{\beta}(t)=\mathrm{T}_{\mathbf{v}(t)} \mathfrak{i}\left(\mathrm{T}_{t} \mathbf{v}(1)\right), 4
$$

where $\dot{\lambda}(t) \in \mathbb{R}$ and $\dot{v}_{\alpha}(t) \in W_{\alpha}^{\min }\left(\mathbf{v}_{0}\right)$ for $t \in I$ and $\alpha \in D$. Moreover, if we assume that for each $\alpha \in D$, $V_{\alpha}$ is a Hilbert space and $v_{\alpha}(t) \in \mathbb{S}_{V_{\alpha}}$, i.e., $\left\|v_{\alpha}(t)\right\|_{\alpha}=1$ for $t \in I$, then $\dot{v}_{\alpha}(t) \in \mathbb{T}_{v_{\alpha}(t)}\left(\mathbb{S}_{V_{\alpha}}\right)$ for $t \in I$ and $\alpha \in D$.

Proof. First of all, we recall that by the construction of $\mathcal{U}\left(\mathbf{v}_{0}\right)$ it follows that $W_{\alpha}^{\min }\left(\mathbf{v}_{0}\right)=W_{\alpha}^{\min }(\mathbf{v}(t))$ and that $U_{\alpha}^{\min }\left(\mathbf{v}_{0}\right)=\operatorname{span}\left\{v_{0}^{(\alpha)}\right\}$ is linearly isomorphic to $U_{\alpha}^{\min }(\mathbf{v}(t))$ for all $t \in I$ and $\alpha \in D$. Assume $\Theta_{\mathbf{v}_{0}}(\mathbf{v}(t))=\left(\lambda(t), L_{1}(t), \ldots, L_{d}(t)\right)$, i.e.,

$$
\mathbf{v}(t):=\lambda(t) \bigotimes_{\alpha \in D}\left(i d_{\alpha}+L_{\alpha}(t)\right)\left(v_{0}^{(\alpha)}\right)
$$

where $\lambda \in \mathcal{C}^{1}(I, \mathbb{R} \backslash\{0\}), L_{\alpha} \in \mathcal{C}^{1}\left(I, \mathcal{L}\left(U_{\alpha}^{\min }\left(\mathbf{v}_{0}\right), W_{\alpha}^{\min }\left(\mathbf{v}_{0}\right)\right)\right)$ and $\left(i d_{\alpha}+L_{\alpha}(t)\right)\left(v_{0}^{(\alpha)}\right) \in U_{\alpha}^{\min }(\mathbf{v}(t))$ for $\alpha \in D$. We point out that the linear map $\mathrm{T}_{t} \mathbf{v}: \mathbb{R} \rightarrow \mathbb{T}_{\mathbf{v}(t)}\left(\mathfrak{M}_{(1, \ldots, 1)}(\mathbf{V})\right)$ is characterised by

$$
\mathrm{T}_{t} \mathbf{v}(1)=\left(\Theta_{\mathbf{v}_{0}} \circ \mathbf{v}\right)^{\prime}(t)=\left(\dot{\lambda}(t), \dot{L}_{1}(t), \ldots, \dot{L}_{d}(t)\right) .
$$

Since $L_{\alpha} \in \mathcal{C}^{1}\left(I, \mathcal{L}\left(U_{\alpha}^{\min }\left(\mathbf{v}_{0}\right), W_{\alpha}^{\min }\left(\mathbf{v}_{0}\right)\right)\right)$ then $\dot{L}_{\alpha} \in \mathcal{C}^{0}\left(I, \mathcal{L}\left(U_{\alpha}^{\min }\left(\mathbf{v}_{0}\right), W_{\alpha}^{\min }\left(\mathbf{v}_{0}\right)\right)\right)$. Observe that $U_{\alpha}^{\min }\left(\mathbf{v}_{0}\right)$ and $U_{\alpha}^{\min }(\mathbf{v}(t))$ have $W_{\alpha}^{\min }\left(\mathbf{v}_{0}\right)$ as a common complement. From Lemma 2.10 we know that

$$
\left.P_{U_{\alpha}^{\min }\left(\mathbf{v}_{0}\right) \oplus W_{\alpha}^{\min }\left(\mathbf{v}_{0}\right)}\right|_{U_{\alpha}^{\min }(\mathbf{v}(t))}: U_{\alpha}^{\min }(\mathbf{v}(t)) \longrightarrow U_{\alpha}^{\min }\left(\mathbf{v}_{0}\right)
$$

is a linear isomorphism. We can write

$$
L_{\alpha}(t)=L_{\alpha}(t) P_{U_{\alpha}^{\min }\left(\mathbf{v}_{0}\right) \oplus W_{\alpha}^{\min }\left(\mathbf{v}_{0}\right)} \text { and } \dot{L}_{\alpha}(t)=\dot{L}_{\alpha}(t) P_{U_{\alpha}^{\min }\left(\mathbf{v}_{0}\right) \oplus W_{\alpha}^{\min }\left(\mathbf{v}_{0}\right)},
$$

and then in (5.5) we identify $\left.\dot{L}_{\alpha}(t) \in \mathcal{L}\left(U_{\alpha}^{\min }\left(\mathbf{v}_{0}\right), W_{\alpha}^{\min }\left(\mathbf{v}_{0}\right)\right)\right)$ with

$$
\left.\left.\dot{L}_{\alpha}(t) P_{U_{\alpha}^{\min }\left(\mathbf{v}_{0}\right) \oplus W_{\alpha}^{\min }\left(\mathbf{v}_{0}\right)}\right|_{U_{\alpha}^{\min }(\mathbf{v}(t))} \in \mathcal{L}\left(U_{\alpha}^{\min }(\mathbf{v}(t)), W_{\alpha}^{\min }\left(\mathbf{v}_{0}\right)\right)\right) .
$$

Introduce $v_{\alpha}(t):=\left(i d_{\alpha}+L_{\alpha}(t)\right)\left(v_{0}^{(\alpha)}\right)$ for $\alpha \in D$. Then

$$
\dot{L}_{\alpha}(t)\left(v_{\alpha}(t)\right)=\left.\dot{L}_{\alpha}(t) P_{U_{\alpha}^{\min }\left(\mathbf{v}_{0}\right) \oplus W_{\alpha}^{\min }\left(\mathbf{v}_{0}\right)}\right|_{U_{\alpha}^{\min }(\mathbf{v}(t))}\left(v_{0}^{(\alpha)}+L_{\alpha}(t)\left(v_{0}^{(\alpha)}\right)\right)=\dot{L}_{\alpha}(t)\left(v_{0}^{(\alpha)}\right)
$$

holds for all $t \in I$ and $\alpha \in D$. Hence

$$
\dot{v}_{\alpha}(t)=\dot{L}_{\alpha}(t)\left(v_{0}^{(\alpha)}\right)=\dot{L}_{\alpha}(t)\left(v_{\alpha}(t)\right)
$$

\footnotetext{
${ }^{4}$ Observe that the derivative at $t$ of a map $\mathbf{v}: I \rightarrow \mathfrak{M}_{(1, \ldots, 1)}(\mathbf{V})$ considered as a morphism between manifolds is given by a linear map $\mathrm{T}_{t} \mathbf{v}: \mathbb{R} \rightarrow \mathbb{T}_{\mathbf{v}(t)}\left(\mathfrak{M}_{(1, \ldots, 1)}(\mathbf{V})\right)$ which is characterised by the fact that $\mathrm{T}_{t} \mathbf{v}(\dot{\mu})=\dot{\mu} \mathrm{T}_{t} \mathbf{v}(1)$ holds for all $\dot{\mu} \in \mathbb{R}$. It allows us to identify the linear map $\mathrm{T}_{t} \mathbf{v}$ with the vector $\mathrm{T}_{t} \mathbf{v}(1)$, that represents the derivative of the curve $\mathbf{v}(t)$ by using local coordinates which is usually written as $\dot{\mathbf{v}}(t)$ by abuse of notation.
} 
holds for all $t \in I$ and $\alpha \in D$. From Lemma 4.6(b) and (5.5), we have

$$
\mathrm{T}_{\mathbf{v}(t)} \mathfrak{i}\left(\mathrm{T}_{t} \mathbf{v}(1)\right)=\dot{\lambda}(t) \bigotimes_{\alpha \in D} v_{\alpha}(t)+\lambda(t) \sum_{\alpha \in D} \dot{L}_{\alpha}(t)\left(v_{\alpha}(t)\right) \otimes \bigotimes_{\beta \neq \alpha} v_{\beta}(t),
$$

and, by using (5.6) for $\mathbf{v}(t)=\lambda(t) \bigotimes_{\alpha \in D} v_{\alpha}(t)$, we obtain (5.4).

To prove the second statement, recall that $U_{\alpha}^{\min }(\mathbf{v}(t))=\operatorname{span}\left\{v_{\alpha}(t)\right\}$ and $V_{\alpha}=U_{\alpha}^{\min }(\mathbf{v}(t)) \oplus W_{\alpha}^{\min }\left(\mathbf{v}_{0}\right)$ for $\alpha \in D$. Let $\langle\cdot, \cdot\rangle_{\alpha}$ be the scalar product defined on $V_{\alpha}(\alpha \in D)$. Then we consider

$$
W_{\alpha}^{\min }\left(\mathbf{v}_{0}\right)=\operatorname{span}\left\{v_{\alpha}(t)\right\}^{\perp}=\left\{u_{\alpha} \in V_{\alpha}:\left\langle u_{\alpha}, v_{\alpha}(t)\right\rangle_{\alpha}=0\right\} \text { for } \alpha \in D
$$

and hence $\left.\left\langle\dot{v}_{\alpha}(t)\right), v_{\alpha}(t)\right\rangle_{\alpha}=0$ holds for $\alpha \in D$. From Remark 2.29, we have $\left(\dot{v}_{1}(t), \ldots, \dot{v}_{d}(t)\right) \in \mathcal{C}\left(I, \times_{\alpha \in D} \mathbb{T}_{v_{\alpha}(t)}\left(\mathbb{S}_{V_{\alpha}}\right)\right)$, because $W_{\alpha}^{\min }\left(\mathbf{v}_{0}\right)=\mathbb{T}_{v_{\alpha}(t)}\left(\mathbb{S}_{V_{\alpha}}\right)$ for $\alpha \in D$.

The next result, where we assume that $\lambda(t)=\lambda_{0}=1$ holds for all time $t$, gives us the time dependent Hartree method on tensor Banach (not necessarily Hilbert) spaces (compare with Theorem 3.1 in [27).

Theorem 5.6 (Time dependent Hartree method on tensor Banach spaces) The solution $\mathbf{v}_{r}(t)=$ $\bigotimes_{\alpha \in D} v_{\alpha}(t)$, with $\left(v_{1}(t), \ldots, v_{d}(t)\right) \in \times_{\alpha \in D} V_{\alpha}$, of

$$
\begin{aligned}
\dot{\mathbf{v}}_{r}(t) & =\mathcal{P}_{\mathbf{v}_{r}(t)}\left(A \mathbf{v}_{r}(t)\right) \text { for } t \in I, \\
\mathbf{v}_{r}(0) & =\mathbf{v}_{0},
\end{aligned}
$$

satisfies

$$
\left\langle\dot{w}_{\alpha} \otimes\left(\bigotimes_{\substack{\beta \in D \\ \beta \neq \alpha}} v_{\beta}(t)\right), J\left(\dot{\mathbf{v}}_{r}(t)-A \mathbf{v}_{r}(t)\right)\right\rangle=0 \quad \text { for all } \dot{w}_{\alpha} \in V_{\alpha}, \quad \alpha \in D .
$$

Proof. From Lemma [5.5 we have $\mathbb{T}_{\mathbf{v}_{r}(t)}\left(\mathfrak{M}_{(1, \ldots, 1)}(\mathbf{V})\right)=\mathbb{R} \times \times_{\alpha \in D} W_{\alpha}^{\min }\left(\mathbf{v}_{0}\right)$, Thus, for each $\dot{\mathbf{w}} \in$ $\mathrm{T}_{\mathbf{v}(t)} i\left(\mathbb{T}_{\mathbf{v}(t)}\left(\mathfrak{M}_{(1, \ldots, 1)}(\mathbf{V})\right)\right)$ there exists $\left(\dot{\varpi}, \dot{w}_{1}, \ldots, \dot{w}_{d}\right) \in \mathbb{R} \times \times_{\alpha \in D} W_{\alpha}^{\min }\left(\mathbf{v}_{0}\right)$, such that

$$
\dot{\mathbf{w}}=\dot{\varpi} \bigotimes_{\alpha \in D} v_{\alpha}(t)+\sum_{\alpha \in D} \dot{w}_{\alpha} \otimes\left(\bigotimes_{\substack{\beta \in D \\ \beta \neq \alpha}} v_{\beta}(t)\right)
$$

Observe that (5.3) holds if and only if

$$
\left\langle\dot{\varpi} \bigotimes_{\alpha \in D} v_{\alpha}(t)+\sum_{\alpha \in D} \dot{w}_{\alpha} \otimes\left(\bigotimes_{\substack{\beta \in D \\ \beta \neq \alpha}} v_{\beta}(t)\right), J\left(\dot{\mathbf{v}}_{r}(t)-A \mathbf{v}_{r}(t)\right)\right\rangle=0
$$

for all $\left(\dot{\varpi}, \dot{w}_{1}, \ldots, \dot{w}_{d}\right) \in \mathbb{R} \times \times_{\alpha \in D} W_{\alpha}^{\min }\left(\mathbf{v}_{\mathbf{0}}\right)$. In particular, for a fixed $\alpha \in D$ take $\dot{w}_{\beta}=0$ for all $\beta \neq \alpha$ and $\dot{\varpi}=0$ then

$$
\left\langle\dot{w}_{\alpha} \otimes\left(\bigotimes_{\substack{\beta \in D \\ \beta \neq \alpha}} v_{\beta}(t)\right), J\left(\dot{\mathbf{v}}_{r}(t)-A \mathbf{v}_{r}(t)\right)\right\rangle=0
$$

holds for all $\dot{w}_{\alpha} \in W_{\alpha}^{\min }\left(\mathbf{v}_{\mathbf{0}}\right)$. By taking $\dot{\varpi}=1$ and $\dot{w}_{\beta}=0$ for all $\beta \in D$ it holds

$$
\left\langle\bigotimes_{\alpha \in D} v_{\alpha}(t), J\left(\dot{\mathbf{v}}_{r}(t)-A \mathbf{v}_{r}(t)\right)\right\rangle=0
$$

Since $U_{\alpha}^{\min }(\mathbf{v}(t))=\operatorname{span}\left\{v_{\alpha}(t)\right\}$ and $V_{\alpha}=U_{\alpha}^{\min }(\mathbf{v}(t)) \oplus W_{\alpha}^{\min }\left(\mathbf{v}_{0}\right)$ for $\alpha \in D$ the theorem follows.

Let $\langle\cdot, \cdot\rangle_{\alpha}$ be a scalar product defined on $V_{\alpha}(\alpha \in D)$, i.e., $V_{\alpha}$ is a pre-Hilbert space. Then $\mathbf{V}={ }_{a} \bigotimes_{\alpha \in D} V_{\alpha}$ is again a pre-Hilbert space with a scalar product which is defined for elementary tensors $\mathbf{v}=\bigotimes_{\alpha \in D} v^{(\alpha)}$ and $\mathbf{w}=\bigotimes_{\alpha \in D} w^{(\alpha)}$ by

$$
\langle\mathbf{v}, \mathbf{w}\rangle=\left\langle\bigotimes_{\alpha \in D} v^{(\alpha)}, \bigotimes_{\alpha \in D} w^{(\alpha)}\right\rangle:=\prod_{\alpha \in D}\left\langle v^{(\alpha)}, w^{(\alpha)}\right\rangle_{\alpha} \quad \text { for all } v^{(\alpha)}, w^{(\alpha)} \in V_{\alpha} .
$$


This bilinear form has a unique extension $\langle\cdot, \cdot\rangle: \mathbf{V} \times \mathbf{V} \rightarrow \mathbb{R}$. One verifies that $\langle\cdot, \cdot\rangle$ is a scalar product, called the induced scalar product. Let $\mathbf{V}$ be equipped with the norm $\|\cdot\|$ corresponding to the induced scalar product $\langle\cdot, \cdot\rangle$. As usual, the Hilbert tensor space $\mathbf{V}_{\|\cdot\|}=\|\cdot\| \bigotimes_{\alpha \in D} V_{\alpha}$ is the completion of $\mathbf{V}$ with respect to $\|\cdot\|$. Since the norm $\|\cdot\|$ is derived via (5.7), it is easy to see that $\|\cdot\|$ is a reasonable and even uniform crossnorm. Moreover, without loss of generality, we can assume $\left\|v_{0}^{(\alpha)}\right\|_{\alpha}=1$ for $\alpha \in D$.

Before stating the next result, we introduce for $\mathbf{v}_{r}(t)=\lambda(t) \bigotimes_{\alpha \in D} v_{\alpha}(t)$ the following time dependent bilinear forms

$$
\mathrm{a}_{\alpha}(t ; \cdot, \cdot): V_{\alpha} \times V_{\alpha} \longrightarrow \mathbb{R}
$$

defined by

$$
\mathrm{a}_{\alpha}\left(t ; z_{\alpha}, y_{\alpha}\right):=\left\langle A\left(z_{\alpha} \otimes \bigotimes_{\substack{\beta \in D \\ \beta \neq \alpha}} v_{\beta}(t)\right),\left(y_{\alpha} \otimes \bigotimes_{\substack{\beta \in D \\ \beta \neq \alpha}} v_{\beta}(t)\right)\right\rangle
$$

for each $\alpha \in D$. Now, we will show the next result (compare with Theorem 3.1 in [27]).

Theorem 5.7 (Time dependent Hartree method on tensor Hilbert spaces) The solution $\mathbf{v}_{r}(t)=$ $\lambda(t) \bigotimes_{\alpha \in D} v_{\alpha}(t)$ with $\left(v_{1}(t), \ldots, v_{d}(t)\right) \in \times_{\alpha \in D} \mathbb{S}_{V_{\alpha}}$ of

$$
\begin{aligned}
\dot{\mathbf{v}}_{r}(t) & =\mathcal{P}_{\mathbf{v}_{r}(t)}\left(A \mathbf{v}_{r}(t)\right) \text { for } t \in I, \\
\mathbf{v}_{r}(0) & =\mathbf{v}_{0},
\end{aligned}
$$

satisfies

$$
\left\langle\dot{v}_{\alpha}(t), \dot{w}_{\alpha}\right\rangle_{\alpha}-\mathrm{a}_{\alpha}\left(t ; v_{\alpha}(t), \dot{w}_{\alpha}\right)=0 \quad \text { for all } \dot{w}_{\alpha} \in \mathbb{T}_{v_{\alpha}(t)}\left(\mathbb{S}_{V_{\alpha}}\right), \quad \alpha \in D,
$$

and

$$
\lambda(t)=\lambda_{0} \exp \left(\int_{0}^{t}\left\langle A\left(\otimes_{\alpha \in D} v_{\alpha}(s)\right), \otimes_{\alpha \in D} v_{\alpha}(s)\right\rangle d s\right) .
$$

Proof. From Lemma 5.5 we have $\mathbb{T}_{\mathbf{v}_{r}(t)}\left(\mathfrak{M}_{(1, \ldots, 1)}(\mathbf{V})\right)=\mathbb{R} \times \times_{\alpha \in D} \mathbb{T}_{v_{\alpha}(t)}\left(\mathbb{S}_{V_{\alpha}}\right)$, Thus, for each $\dot{\mathbf{w}} \in$ $\mathrm{T}_{\mathbf{v}(t)} i\left(\mathbb{T}_{\mathbf{v}(t)}\left(\mathfrak{M}_{(1, \ldots, 1)}(\mathbf{V})\right)\right)$ there exists $\left(\dot{\varpi}, \dot{w}_{1}, \ldots, \dot{w}_{d}\right) \in \mathbb{R} \times \times_{\alpha \in D} \mathbb{T}_{v_{\alpha}(t)}\left(\mathbb{S}_{V_{\alpha}}\right)$, such that

$$
\dot{\mathbf{w}}=\dot{\varpi} \bigotimes_{\alpha \in D} v_{\alpha}(t)+\lambda(t) \sum_{\alpha \in D} \dot{w}_{\alpha} \otimes\left(\bigotimes_{\substack{\beta \in D \\ \beta \neq \alpha}} v_{\beta}(t)\right)
$$

Then (5.3) holds if and only if

$$
\left\langle\dot{\varpi} \bigotimes_{\alpha \in D} v_{\alpha}(t)+\lambda(t) \sum_{\alpha \in D} \dot{w}_{\alpha} \otimes\left(\bigotimes_{\substack{\beta \in D \\ \beta \neq \alpha}} v_{\beta}(t)\right), \dot{\mathbf{v}}_{r}(t)-A \mathbf{v}_{r}(t)\right\rangle=0
$$

for all $\left(\dot{\varpi}, \dot{w}_{1}, \ldots, \dot{w}_{d}\right) \in \mathbb{R} \times \times_{\alpha \in D} \mathbb{T}_{v_{\alpha}(t)}\left(\mathbb{S}_{V_{\alpha}}\right)$. Then

$$
\begin{array}{r}
\dot{\lambda}(t) \dot{\varpi}+\lambda(t)^{2} \sum_{\alpha \in D}\left(\left\langle\dot{v}_{\alpha}(t), \dot{w}_{\alpha}\right\rangle_{\alpha}-\left\langle A \bigotimes_{\mu \in D} v_{\mu}(t), \dot{w}_{\alpha} \otimes\left(\bigotimes_{\substack{\beta \in D \\
\beta \neq \alpha}} v_{\beta}(t)\right)\right\rangle\right) \\
-\lambda(t) \dot{\varpi}\left\langle A \bigotimes_{\alpha \in D} v_{\alpha}(t), \bigotimes_{\alpha \in D} v_{\alpha}(t)\right\rangle=0,
\end{array}
$$

i.e.,

$$
\begin{aligned}
& \dot{\varpi}\left(\dot{\lambda}(t)-\lambda(t)\left\langle A \bigotimes_{\alpha \in D} v_{\alpha}(t), \bigotimes_{\alpha \in D} v_{\alpha}(t)\right\rangle\right) \\
& +\lambda(t)^{2} \sum_{\alpha \in D}\left(\left\langle\dot{v}_{\alpha}(t), \dot{w}_{\alpha}\right\rangle_{\alpha}-\left\langle A \bigotimes_{\mu \in D} v_{\mu}(t), \dot{w}_{\alpha} \otimes\left(\bigotimes_{\substack{\beta \in D \\
\beta \neq \alpha}} v_{\beta}(t)\right)\right\rangle\right)=0
\end{aligned}
$$


holds for all $\dot{\varpi} \in \mathbb{R}$ and $\left(\dot{w}_{1}, \ldots, \dot{w}_{d}\right) \in \times_{\alpha \in D} \mathbb{T}_{v_{\alpha}(t)}\left(\mathbb{S}_{V_{\alpha}}\right)$. If $\lambda(t)$ solves the differential equation

$$
\begin{aligned}
\dot{\lambda}(t) & =\left\langle A\left(\otimes_{\alpha \in D} v_{\alpha}(t)\right), \otimes_{\alpha \in D} v_{\alpha}(t)\right\rangle \lambda(t) \\
\lambda(0) & =\lambda_{0},
\end{aligned}
$$

i.e.,

$$
\lambda(t)=\lambda_{0} \exp \left(\int_{0}^{t}\left\langle A\left(\otimes_{\alpha \in D} v_{\alpha}(s)\right), \otimes_{\alpha \in D} v_{\alpha}(s)\right\rangle d s\right),
$$

then the first term of (5.8) is equal to 0 . Therefore, from (5.8) we obtain that for all $\alpha \in D$,

$$
\left\langle\dot{v}_{\alpha}(t), \dot{w}_{\alpha}\right\rangle_{\alpha}-\left\langle A \bigotimes_{\mu \in D} v_{\mu}(t), \dot{w}_{\alpha} \otimes\left(\bigotimes_{\substack{\beta \in D \\ \beta \neq \alpha}} v_{\beta}(t)\right)\right\rangle=0
$$

that is,

$$
\left\langle\dot{v}_{\alpha}(t), \dot{w}_{\alpha}\right\rangle_{\alpha}-\mathrm{a}_{\alpha}\left(t ; v_{\alpha}(t), \dot{w}_{\alpha}\right)=0
$$

holds for all $\dot{w}_{\alpha} \in \mathbb{T}_{v_{\alpha}(t)}\left(\mathbb{S}_{V_{\alpha}}\right)$, and the theorem follows.

\subsection{Concluding Remarks}

We would point out that when we assume that $V_{\alpha}=V$ for all $\alpha \in D$ then the theory presented above covers the classical MCTDH approximation for molecules (see for example Section 1.9 in [21]). In fact the approximate wave function $\mathbf{v}_{r}(t)$ computed on $\mathfrak{M}_{(1, \ldots, 1)}(\mathbf{V})$ does not conform the Pauli's exclusion principle. To take into account the antisymmetry of the wave function we need to use the so-called multi-configuration time-dependent Hartree-Fock (MCTDHF) approximation. The MCTDHF is based on the use of the socalled Hartree-Fock manifold. This manifold is constructed by using the existence of a projection $P_{\mathfrak{S}}$ from $\mathbf{V}$ to the linear subspace of antisymmetric tensors of $\mathbf{V}$ (corresponding to fermions). Then the Hartree-Fock manifold is defined as

$$
\mathfrak{M}_{(1, \ldots, 1)}^{\mathcal{A}}(\mathbf{V}):=\left\{P_{\mathfrak{S}}\left(v_{1} \otimes \cdots \otimes v_{d}\right): v_{\alpha} \in V_{\alpha}, \alpha \in D\right\} .
$$

In a similar way, the use of a projection onto the linear subspace of symmetric tensors of $\mathbf{V}$ (corresponding to bosons) allows us to introduce a manifold, namely $\mathfrak{M}_{(1, \ldots, 1)}^{\mathfrak{S}}(\mathbf{V})$. The extension of the results given in this paper to $\mathfrak{M}_{(1, \ldots, 1)}^{\mathfrak{A}}(\mathbf{V})$ and $\mathfrak{M}_{(1, \ldots, 1)}^{\mathfrak{S}}(\mathbf{V})$ are part of a work in progress and will be published elsewhere.

\section{References}

[1] P. A. Absil, R. Mahoni, and R. Sepulchre, Optimization Algorithms on Matrix Manifolds, Princeton University Press, 2008.

[2] Y. I. Alber, James orthogonality and orthogonal decompositions of Banach spaces. J. Math. Anal. Appl. 312 (2005), 330-342.

[3] A. Arnold and T. Jahnke, On the approximation of high-dimensional differential equations in the hierarchical Tucker format. BIT Numer. Math., 54 (2014), 305-341.

[4] C. Bardos, I .Catto, N. Mauser and S. Trabelsi, Setting and Analysis of the multiconfiguration timedependent Hartree-Fock equations, Arch. Rational Mech. Anal. 198 Issue 1 (2010), 273-330.

[5] M. S. Berger, Nonlinearity and Functional Analysis. Lectures on Nonlinear Problems in Mathematical Analysis. Academic Press, 1997.

[6] D. Belita, Smooth homogeneous structures in operator theory. Chapman \& Hall/CRC, 2006.

[7] I. Cioranescu, Geometry of Banach Spaces, Duality Mappings and Nonlinear Problems. Springer-Verlag, 1990. 
[8] G. Dirr, V. Rakocevic, and H. K. Wimmer, Estimates for projections in Banach spaces and existence of direct complements. Studia Math., 170:2 (2005), 211-216.

[9] A. Douady, Le problème des modules pour les sous-espaces analytiques compacts d'un espace analytique donné. Annales de l'Institut Fourier, 16 (1) (1966), 1-95.

[10] M. Fabian, P. Habala, P. Hajek, and V. Montesinos, Banach Space Theory. Springer-Verlag, 2011.

[11] A. Falcó and W. Hackbusch, Minimal subspaces in tensor representations. Found. Comput. Math. 12 (2012), 765-803.

[12] A. Falcó and A. Nouy, Proper generalized decomposition for nonlinear convex problems in tensor Banach spaces. Numer. Math. 121 (2012), 503-530.

[13] K. Floret, Weakly compact sets, Lect. Notes Math., vol.119. Springer-Verlag, 1980.

[14] W. H. Greub, Linear Algebra. Graduate Text in Mathematics, 4th ed., Springer-Verlag, 1981.

[15] A. Grothendieck, Résumé de la théorie métrique des produit tensoriels topologiques. Bol. Soc. Mat. São Paulo 8 (1953/56), 1-79.

[16] E. Hairer, C. Lubich, and G. Wanner, Geometrical Numerical Integration: Structure-Preserving Algorithms for Ordinary Differential Equations, 2nd ed., Springer-Verlag, 2006.

[17] J. Haegeman, M. Mariën, T. J. Osborne, and F. Verstraete, Geometry of matrix product states: Metric, parallel transport, and curvature. Journal of Mathematical Physics 55, 021902 (2014).

[18] W. Hackbusch and S. Kühn, A new scheme for the tensor representation. J. Fourier Anal. Appl. 15 (2009), 706-722.

[19] W. Hackbusch, Tensor spaces and numerical tensor calculus. Springer-Verlag, 2012.

[20] P. Hájek and M. Johanis, Smooth analysis in Banach spaces. Series in Non-linear analysis and applications 19, Walter de Gruyter, 2014.

[21] D. R. Hartree, The calculation of atomic structures. Chapman \& Hall, 1957.

[22] S. Holtz, Th. Rohwedder, and R. Schneider, On manifold of tensors of fixed TT rank. Numer. Math. 121 (2012), 701-731.

[23] S. Kamimura and W. Takahashi, Strong convergence of a proximal-type algorithm in a Banach space. SIAM J. Optim. 13 (2003), 938-945.

[24] O. Koch and C. Lubich, Dynamical tensor approximation. SIAM J. Matrix Anal. Appl. 31 (2010), $2360-2375$.

[25] S. Lang, Differential and Riemannian Manifolds. Graduate Texts in Mathematics 160, Springer-Verlag, 1995.

[26] W. A. Light and E. W. Cheney, Approximation theory in tensor product spaces. Lect. Notes Math. 1169, Springer-Verlag, 1985.

[27] C. Lubich, From Quantum to Classical Molecular Dynamics: Reduced Models and Numerical Analysis. European Mathematical Society, 2008.

[28] J. E. Marsden, T. Ratiu, and R. Abraham, Manifolds, Tensor Analysis, and Applications. SpringerVerlag, 1988.

[29] B. Simon, Uniform crossnorms. Pacific J. Math. 46 (1973), 555-560.

[30] I. V. Oseledets, A new tensor decomposition. Doklady Math. 80 (2009), 495-496.

[31] I. V. Oseledets, Tensor-train decomposition. SIAM J. Sci. Comput. 33 (2011), 2295-2317. 
[32] I. V. Oseledets and E. E. Tyrtyshnikov, TT-cross approximation for multidimensional arrays. Linear Algebra Appl. 432 (2010), 70-88.

[33] E. Schmidt, Zur Theorie der linearen und nichtlinearen Integralgleichungen I, Math. Ann. 63 (1906), 433-476.

[34] H. Upmeier, Symmetric Banach manifolds and Jordan $C^{*}$ Algebras. North-Holland, 1985.

[35] A. Uschmajew and B. Vandereycken, The geometry of algorithms using hierarchical tensors. Linear Algebra and its Applications, Volume 439, Issue 1, (2013) 133-166.

[36] F. Verstraete and J. I. Cirac, Matrix product states represent ground states faithfully. Phys. Rev. B Condens. Matter Mater. Phys. 73, 094423 (2006).

[37] G. Vidal, Efficient classical simulation of slightly entangled quantum computations. Phys. Rev. Lett. 91 (14), 147902 (2003). 